\title{
Combinatorial tumor suppressor inactivation efficiently initiates lung adenocarcinoma
} with therapeutic vulnerabilities

Maryam Yousefi ${ }^{1,8^{*}}$, Gábor Boross ${ }^{4,8}$, Carly Weiss ${ }^{4}$, Christopher W. Murray ${ }^{2}$, Jess D. Hebert ${ }^{1}$,

8 Hongchen $\mathrm{Cai}^{1}$, Emily L. Ashkin ${ }^{2}$, Saswati Karmakar ${ }^{1}$, Laura Andrejka ${ }^{1}$, Leo Chen ${ }^{1}$, Minwei

9 Wang ${ }^{1}$, Min K. Tsai ${ }^{1}$, Wen-Yang Lin ${ }^{1}$, Chuan $\mathrm{Li}^{4}$, Pegah Yakhchalian ${ }^{7}$, Caterina I. Colón ${ }^{2}$, Su-

10 Kit Chew ${ }^{5,6}$, Pauline $\mathrm{Chu}^{3}$, Charles Swanton ${ }^{5,6}$, Christian A. Kunder ${ }^{3}$, Dmitri A. Petrov ${ }^{2,4}$, Monte

11 M. Winslow ${ }^{1,2,3 *}$

$13{ }^{1}$ Department of Genetics, Stanford University School of Medicine, Stanford, CA, USA

$14{ }^{2}$ Cancer Biology Program, Stanford University School of Medicine, Stanford, CA, USA

$15{ }^{3}$ Department of Pathology, Stanford University School of Medicine, Stanford, CA, USA

$16{ }^{4}$ Department of Biology, Stanford University, Stanford, CA, USA

$17{ }^{5}$ Cancer Evolution and Genome Instability Laboratory, University College London Cancer Institute, London, UK

$18{ }^{6}$ Cancer Evolution and Genome Instability Laboratory, The Francis Crick Institute, London, UK

$19{ }^{7}$ Department of Medicine, David Geffen School of Medicine at University of California, Los Angeles, Los Angeles,

20 CA, USA

$21{ }^{8}$ These authors contributed equally

22

* Corresponding authors:

Monte M. Winslow, Stanford University School of Medicine | 279 Campus Drive, Beckman Center B256, Stanford, CA 94305. Phone: 650-725-8696 | Fax: 650-725-1534 E-mail: mwinslow@ stanford.edu

28 CA 94305. Phone: (650) 725-2182| E-mail: yousefi@ stanford.edu 


\section{ABSTRACT}

Lung cancer is the leading cause of cancer death worldwide, with lung adenocarcinoma

38 being the most common subtype. Many oncogenes and tumor suppressor genes are altered in this

39 cancer type and the discovery of oncogene mutations has led to the development of targeted

40 therapies that have improved clinical outcomes. However, a large fraction of lung

41 adenocarcinomas lacks mutations in known oncogenes, and the genesis and treatment of these

42 oncogene-negative tumors remain enigmatic. Here, we perform iterative in vivo functional

43 screens using quantitative autochthonous mouse model systems to uncover the genetic and

44 biochemical changes that enable efficient lung tumor initiation in the absence of oncogene

45 alterations. Through the generation of hundreds of diverse combinations of tumor suppressor

46 alterations, we demonstrate that the inactivation of suppressors of the RAS and PI3K pathways

47 drive the development of oncogene-negative lung adenocarcinoma. Human genomic data and

48 histology identified RAS/MAPK and PI3K pathway activation as a common event in oncogene-

49 negative human lung adenocarcinomas. We demonstrate that these Onc-negative ${ }^{\text {RAS/PI3K }}$ tumors

50 and related cell lines are vulnerable to pharmacological inhibition of these signaling axes. These

51 results transform our understanding of this prevalent yet understudied subtype of lung

52 adenocarcinoma.

\section{INTRODUCTION}

55 Lung cancer is the leading cause of cancer death ${ }^{1}$. Lung adenocarcinoma, the most

56 prevalent subtype of lung cancer, has frequent alterations in receptor tyrosine kinase and

57 RAS/RAF pathway oncogenes, including mutations in EGFR and $K R A S^{2}$. The identification of

58 driver oncogenes has enabled a shift from toxic chemotherapies to less toxic and more effective 
59 therapies that often target the oncogenes ${ }^{3}$. However, approximately 30 percent of lung

60 adenocarcinomas are thought to lack a driving oncogene ${ }^{4-6}$. Consequently, developing targeted

61 therapies for these tumors remains a major unmet challenge for precision thoracic oncology.

62 Extensive genomic and transcriptomic studies suggest that neither technical reasons nor

63 the presence of novel oncogenes likely explain this large and clinically significant population of

64 lung cancer patients $1,2,4-12$. Thus, despite the diagnosis of more than 150,000 patients per year

65 with oncogene-negative lung adenocarcinomas worldwide, the genetic events and biochemical

66 pathway changes that drive the initiation and growth of these tumors remain almost entirely

67 unknown.

Oncogenes and tumor suppressor genes are parts of signaling networks that generate and

69 sustain the biochemical changes that drive tumor initiation and growth ${ }^{13-16}$. Combinatorial

70 alterations in tumor suppressor genes could co-operate to activate pathways driving oncogene-

71 negative lung tumors. Human lung adenocarcinoma have complex patterns of mutations across

72 many putative tumor suppressor genes ${ }^{4}$. However, the ability to predict which combinations of

73 genomic alterations drive cancer in the absence of oncogene activation based on human genomic

74 data alone remains challenging. While human genomic data can predict combinations of

75 genomic mutations as likely cancer drivers when the mutations co-occur at very high frequencies

$76^{17-20}$, identifying pathogenic combinations of less frequently mutated genes poses a nearly

77 insurmountable statistical challenge. Furthermore, the large numbers of mutations in lung

78 cancers, non-genomic mechanism that often inactivate tumor suppressor genes, and generation of

79 similar biochemical effects through inactivation of different genes further reduce the ability of

80 human cancer genomic studies to identify combinatorial alterations that activate driver pathways

81 in lung cancer ${ }^{21-24}$. 
Functional genomic studies within autochthonous cancer models can help identify the

83 pathways involved in tumorigenesis in vivo ${ }^{25}$. Here, we leveraged quantitative mouse model

84 systems to assess the ability of hundreds of combinatorial alterations of tumor suppressor genes,

85 acting across many different signaling pathways, to generate oncogene-negative lung

86 adenocarcinomas in vivo. We uncover pathway-level changes that drive lung cancer in the

87 absence of oncogene mutations, translate these findings to human oncogene-negative lung

88 adenocarcinoma, and leveraged these results to identify therapeutic vulnerabilities.

\section{RESULTS}

A large fraction of human lung adenocarcinomas lack oncogene mutations

To better understand the genomics of lung adenocarcinomas that lack oncogene

94 mutations, we analyzed data from The Cancer Genome Atlas (TCGA) and AACR Genomics

95 Evidence Neoplasia Information Exchange (GENIE) ${ }^{26,27}$. We classified tumors as oncogene-

96 positive if they had high-confidence oncogenic alterations in previously described proto-

97 oncogenes, oncogene-indeterminate if they had alterations of unknown significance in known

98 proto-oncogenes, and oncogene-negative if they had no alterations in known proto-oncogenes

99 (Methods). Consistent with previous publications, we found that 17-18\% of lung

100 adenocarcinomas were oncogene-negative (Figure 1a and S1a) ${ }^{28-30}$. Additionally, 15-27\% of

101 lung adenocarcinomas were oncogene-indeterminate and thus 32-45\% of lung adenocarcinomas

102 lack known oncogene mutations. Patients with oncogene-negative, oncogene-indeterminate, and

103 oncogene-positive lung adenocarcinomas have broadly similar mutational burden and clinical

104 characteristics (Figure S1b-e). 


\section{Combinatorial tumor suppressor gene inactivation enables lung tumor development}

106

107

108

109

110

111

112

113

114

115

To determine whether combinatorial tumor suppressor gene inactivation can drive lung tumor initiation in the absence of oncogene activation, we coupled Cre/loxP-based genetically engineered mouse models and somatic CRISPR/Cas9-based genome editing with tumor barcoding and high-throughput barcode sequencing (Tuba-seq) ${ }^{31-35}$. We used Cre/loxP to

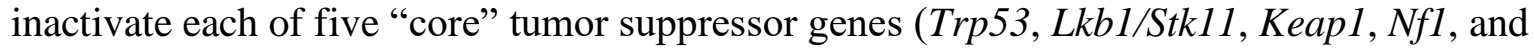
Pten). These genes are within diverse pathways and are frequently inactivated in human lung cancers, including oncogene-negative lung adenocarcinomas (Figure S2a-b) [35-38]. We used CRISPR/Cas9 to coincidentally inactivate panels of additional tumor suppressor genes in lung epithelial cells in mice with floxed alleles of each of the "core" tumor suppressors, a Cre-reporter allele $\left(R 26^{L S L-T o m}(T)^{36}\right)$, and a Cre-regulated $\operatorname{Cas} 9$ allele $\left(H 11^{L S L-C a s 9}(C)^{37}\right)$.

We transduced Nf1 fff $; T C, P t e n^{f f f} ; T C, T r p 53^{f f f} ; T C, L k b 1^{f f f} ; T C$, Keapl ${ }^{f f f} ; T C, T C$, and T mice with two pools of barcoded Lenti-sgRNA/Cre vectors that target $\sim 50$ putative tumor suppressor

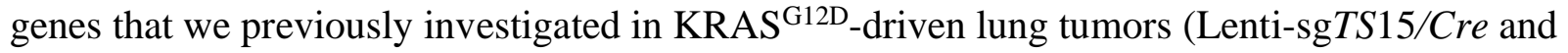
Lenti-sgTS102/Cre) (Figure 1b, S2c-d, S3a, and Table S1) ${ }^{31,32,35}$. The mutation frequency of these genes varied, and mutations in some were enriched in oncogene-negative human lung adenocarcinomas (Table S1) (Figure S2c-d). The combination of Cre/LoxP and CRISPR/Cas9based genome editing should generate hundreds of combinations of genomic alterations in lung epithelial cells. We previously found that a small percent of lung tumors initiated with LentisgRNA/Cre vectors in other lung cancer models contained multiple sgRNAs, consistent with the transduction of the initial cell with multiple Lenti-sgRNA/Cre vectors ${ }^{31,32}$. Thus, we used a high titer of the Lenti-sgRNA/Cre pools in these experiments to increases the likelihood of finding higher-order genetic interactions that drive tumorigenesis. 
One year after transduction with the Lenti-sgRNA/Cre pools, Nfflff;C, Pten ${ }^{f / f}$;TC, and

Trp53fff $; T C$ mice developed a modest number of tumors (defined as Tomato ${ }^{\text {positive }}$ expansion $>0.5$

$130 \mathrm{~mm}$ in diameter), while $L k b 1^{f f f} ; T C$ and Keap ${ }^{f f f}$; $T C$ mice rarely developed any tumors (Figure

131 1c-d, S3b-c). Interestingly, $N f f^{f f f} ; T C$, $P t e n^{f f f} ; T C$, and $T r p 53^{f f f} ; T C$, and $T C$ mice transduced with

132 the larger Lenti-sgTS102/Cre pool developed many more tumors than those transduced with the

133 Lenti-sgTS15/Cre pool. These tumors were positive for TTF1/NKX2-1, a marker for lung

134 adenocarcinoma, and negative for P63 and UCHL1, markers for squamous cell and small cell

135 lung cancer, respectively (Figure 1e).

To determine whether these tumors contained spontaneous oncogene mutations, we PCR-

137 amplified and sequenced 10 genomic regions in Kras, Braf, Nras, and Egfr (Figure S3d, Table

138 S2, and Methods) ${ }^{33,38-46}$. Across 29 samples, we detected only one oncogene mutation (a

$139 \mathrm{Kras}^{\mathrm{Gl2V}}$ mutation in a tumor from a Pten ${ }^{f / f} ; \mathrm{TC}$ mouse). Thus, the majority of these tumors arose

140 in the absence of hotspot mutations in these proto-oncogenes. This is consistent with the low

141 mutation rate in mouse models of lung cancer ${ }^{47}$ and suggests that the inactivation of

142 combinations of specific tumor suppressor genes in Nf1 fff;TC, Pten ${ }^{f / f} ; T C$, and Trp53fff;TC mice

143 drives the development of lung cancer in vivo. Notably, the overall low number of tumors

144 indicates that inactivation of the "core" tumor suppressor genes alone, and most combinations of

145 tumor suppressor genes tested, are insufficient to generate lung tumors.

147 Identification of top candidate tumor suppressor genes involved in oncogene-negative lung 148 tumor formation

The Lenti-sgRNA/Cre vectors contain two-component barcodes in which an sgID

150 identifies the sgRNA and a random barcode (BC) uniquely tags each clonal tumor. Thus, high 
151 throughput sequencing of the sgID-BC region can identify the sgRNA(s) present in each tumor

152 and quantify the number of cancer cells in each tumor (Figure 1b). To determine which

153 sgRNAs were present in the largest tumors, we PCR-amplified the sgID-BC region from

154 genomic DNA from dissected tumors and performed high-throughput sgID-BC sequencing.

155 Most large tumors contained multiple Lenti-sgRNA/Cre vectors therefore, we calculated the

156 statistical enrichment of each sgRNA based on their relative representation in the dissected

157 tumors (Figure 1f and S4, see Methods).

To further quantify the impact of inactivating each tumor suppressor gene on clonal expansion of lung epithelial cells, we performed tumor barcode sequencing (Tuba-seq) on bulk DNA from one lung lobe from each $N f 1^{f f f} ; T C$, Pten $^{f / f} ; T C, T r p 53^{f f f} ; T C$, and $T C$ mouse (Figure

161 1c). Analysis of the number of cells in clonal expansions further nominated tumor suppressor

162 genes that may contribute to tumor initiation and growth (Figure 1f, and S5). Based on these two

163 analyses, we selected 13 genes for further analysis (Figure 1f). The potential importance of

164 these tumor suppressor genes was often supported by both sgRNAs targeting each gene,

165 consistent with on-target effects. Finally, Lenti-sgPten/Cre enrichment in tumors in Nff fff;TC

166 mice and Lenti-sgNf1/Cre enrichment in tumors in Pten ${ }^{f / f}$;TC mice cross-validate our screen

167 (Figure 1f and S4-5).

172 these tumor suppressor genes and one vector with an inert sgRNA (Lenti-sgTS14/Cre pool;

173 Figure 2a). We targeted each gene with the sgRNA that had the most significant effect on tumor 
174 growth and used five times higher titer of each lentiviral vector per mouse than we used in Lenti-

$175 \mathrm{sg}$ TS102/Cre pool, thus increasing the potential for the transduction of the initial cell with

176 multiple Lenti-sgRNA/Cre vectors.

We initiated tumors with Lenti-sgTS14/Cre in Nf1 fff;TC, Pten ${ }^{f / f} ; T C, T r p 53^{f f f} ; T C, T C$, and

$178 \mathrm{Kras}^{L S L-G 12 D} ; T(K T)$ mice. Less than four months after tumor initiation, several NfI ff; $T C$ and

179 Pten ${ }^{f / f} ; T C$ mice showed signs of extensive tumor burden. These mice developed many more

180 tumors than mice of the same genotypes one year after transduction with the Lenti-sgTS102/Cre

181 (compare Figure 2b-c with Figure 1c-d, and S10c). Thus, this pool of candidate tumor

182 suppressor genes is enriched for those that generate oncogene-negative lung tumors.

We performed Tuba-seq on DNA from bulk tumor-bearing lungs to determine the

184 number and size of tumors with each barcoded Lenti-sgRNA/Cre vector. Inactivation of $N f 1$,

185 Rasal, and Pten most dramatically increased tumor size and/or tumor number across all mouse

186 genotypes (Figure 2d-e, S6a-b, and Methods). Inactivation of some of the other tumor

187 suppressor genes less dramatically but significantly increased tumor size and/or tumor number in

188 a genotype-specific manner. This suggests that additional molecular pathways altered by these

189 tumor suppressor genes may also lead to early epithelial expansions.

191 generated through the inactivation of multiple tumor suppressor genes. Vectors targeting $N f 1$,

192 Rasal, and/or Pten were often present in the largest tumors, and the coincident targeting of $N f 1$,

193 Rasal, and Pten was the most frequent combination (Figure 2f-g, S6c-h). To gain greater insight

194 into the contribution of Nfl, Rasal, and Pten inactivation to the generation of oncogene-negative

195 tumors, we transduced $N f 1^{f f f} ; T C, P t e n^{f f f} ; T C, T r p 53^{f f f} ; T C, T C$, and KT mice with a pool of Lenti-

196 sgRNA/Cre vectors that lacked the vectors targeting Nf1, Rasal, and Pten (Lenti-sgTS11/Cre) 
197 (Figure S7a). Approximately four months after transduction, these mice had many fewer tumors

198 than mice transduced with Lenti-sgTS14/Cre pool (Figure S7b-c and S10c). Tuba-seq analysis

199 confirmed a dramatic decrease in tumor burden relative to mice that received the Lenti-

200 sgTS14/Cre pool (Figure 2h). Thus, the inactivation of Nf1, Rasal, and Pten emerged as the

201 most important contributors to the generation of oncogene-negative lung tumors.

202 Extensive experiments generating single and pairwise inactivation of tumor suppressor

203 genes in individual mice led to the development of very few tumors even after long periods of

204 time (Figure S8-S9). Thus, single and pairwise tumor suppressor gene inactivation is rarely

205 sufficient to generate lung tumors and combinatorial inactivation of three or more tumor

206 suppressor genes increases the efficiency of tumor development and/or accelerates the growth of

207 oncogene-negative lung tumors.

209 Combinatorial inactivation of $\mathbf{N} \mathbf{f 1}$, Rasa1, and Pten drives lung adenocarcinoma

210 development comparably to oncogenic Kras mutation

211 To dissect the higher-order genetic interactions between Nf1, Rasal, and Pten, we

212 transduced $T C$ and $T r p 53^{f f f} ; T C$ mice with a pool of eight lentiviral vectors that would inactivate

213 Nf1, Rasal, and Pten individually, in pairwise combinations, and all three simultaneously (Lenti-

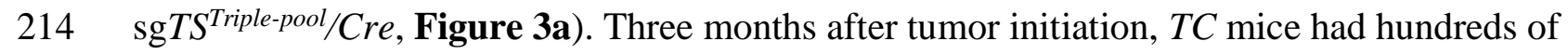

215 large adenomas and adenocarcinomas (Figure 3b-c and Figure S10a-e). Tuba-seq analysis

216 showed that most of the tumor burden arose as a consequence of concomitant inactivation of all

217 three tumor suppressors, with single and pairwise inactivation of these genes generating very few

218 tumors consistent with our previous observations (Figure 3e and S8). Additional inactivation of

$219 \operatorname{Trp53}$ in $\operatorname{Trp5} 53^{\mathrm{fff}}$; $T C$ mice did not increase tumor initiation suggesting that Trp53 is not a major 
suppressor of oncogene-negative lung adenocarcinoma development at these early stages

221 (Figure 3b-f and Figure S10a-h). Finally, to compare the tumor initiation potential of

222 combinatorial Nf1, Rasal, and Pten inactivation with that of a known oncogene, we transduced

$223 K_{r a s}^{L S L-G 12 D} ; T$ mice (which lack Cas9) with Lenti-sgTS Triple-pool/Cre (Figure 3a). Strikingly,

224 coincident inactivation of Nf1, Rasal, and Pten in TC mice was nearly as potent as oncogenic

$225 \mathrm{KRAS}^{\mathrm{G} 12 \mathrm{D}}$ in driving lung tumor initiation in vivo (Figure 3g and Methods).

In molecular evolution studies, generating combinations of genomic alterations and

227 measuring the fitness of each genotype (growth rate) is used to infer the possible and the most

228 probable paths from a wild-type state to a complex genotype ${ }^{48}$. Through the generation of all

229 possible combinatorial alterations of $N f 1$, Rasal, and Pten, we quantified the fitness conferred by

230 each mutation and the relative probabilities of different adaptive paths leading to the triple

231 mutant genotype. Our data suggest that inactivation of these three genes can occur in any order,

232 with each additional alteration further increasing the fitness (Figure 3f). The $N f 1 \rightarrow$ Rasal $\rightarrow$ Pten

233 mutation sequence emerged as the most probable of all six possible paths.

To further analyze tumors driven by inactivation of Nfl, Rasal, and Pten, we initiated

235 tumors in $T C$ and $T r p 53^{f f f}$; $T C$ mice using only the lentiviral vector that targets all three genes

236 (Lenti-sgNf1-sgRasal-sgPten/Cre) (Figure S11a). After only three months, these mice

237 developed very large numbers of lung adenomas and adenocarcinomas (Figure S11b-e). We

238 confirmed the inactivation of $N f 1$, Rasal, and Pten in these tumors and whole-exome sequencing

239 uncovered no putative oncogene mutations and only a few putative tumor suppressor mutations,

240 none of which occurred in more than one tumor (Figure S11f and Table S3). Interestingly, at

241 later timepoints after initiation, tumors in Trp53ff; $T C$ mice progressed to an invasive NKX2- 
$2421^{\text {negative }} \mathrm{HMGA} 2^{\text {positive }}$ state and metastasized to other organs such as liver similar to what has

243 been reported in ras $^{G 12 D}$;Trp53 mutant lung adenocarcinoma models (Figure S12) ${ }^{49}$.

245 Oncogene-negative murine lung adenocarcinomas have activated RAS and PI3K pathways

246 NF1 and RASA1 are negative regulators of RAS, while PTEN is a negative regulator of

247 the PI3K-AKT pathway. Therefore, we investigated the impact of inactivating these tumor

248 suppressor genes on RAS and PI3K pathway activation by immunohistochemistry, as well as by

249 RNA-sequencing (RNA-seq) on FACS-isolated Tomato ${ }^{\text {positive }}$ cancer cells. We generated

250 autochthonous tumors in which Nfl, Rasal, and Pten were inactivated (TC mice with Lenti-

251 sgNf1-sgRasal-sgPten/Cre; Nf1/Rasa1/Pten tumors), KRAS ${ }^{\mathrm{G} 12 \mathrm{D}}$ was expressed $\left(K T ; H 11^{L S L-C a s 9}\right.$

252 mice with Lenti-sgInert/Cre; Kras tumors), or KRAS ${ }^{\mathrm{G} 12 \mathrm{D}}$ was expressed and Pten was

253 inactivated (KT;H1 $1^{L S L-C a s 9}$ mice with Lenti-sgPten/Cre; Kras/Pten tumors) (Figure S13a).

$254 \mathrm{Nf1/Rasa1/Pten}$ tumors had positive staining for pERK (indicative of RAS pathway activation)

255 and pAKT (indicative of PI3K pathway activation) (Figure 4a). Compared with Kras/Pten

256 tumors, the average pERK staining in Nf1/Rasa1/Pten tumors was less intense and pAKT

257 staining was similar (Figure 4b-c). Single-sample gene set variation analysis (ssGSVA) for

258 previously reported gene sets representing RAS and PI3K-AKT regulated genes ${ }^{50,51}$ on our

259 RNA-seq data confirmed that Nf1/Rasa1/Pten tumors had lower RAS pathway gene signature

260 scores than Kras/Pten tumors (Figure S13b). PI3K-AKT pathway gene signature scores were

261 similar in Nf1/Rasa1/Pten and Kras tumors (Figure S13c). The rare tumors that eventually

262 developed after pairwise inactivation of $N f 1$, Rasal, and Pten also had strong activation of RAS

263 and PI3K pathways (Figure S8 and S13d). Based on these analyses, we propose that these 
264 tumors represent a subtype of oncogene-negative lung adenocarcinomas with activated RAS and

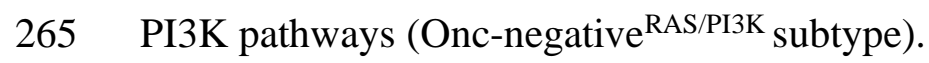

267 Oncogene-negative human lung adenocarcinomas frequently have activation of RAS and

268 PI3K pathways

269 To investigate the activation of RAS and PI3K pathways in human oncogene-negative

270 lung adenocarcinomas, we analyzed oncogene-negative $(\mathrm{N}=35)$ and oncogene-positive $(\mathrm{N}=18)$

271 lung adenocarcinomas. Immunohistochemistry for pERK and pAKT showed that $45 \%$ of

272 oncogene-negative human tumors had moderate to strong activation of both RAS and PI3K

273 pathways and thus represent the Onco-negative ${ }^{R A S / P I 3 K}$ subtype (Figure 4d-h, S13e-j). These

274 tumors were genomically characterized by Stanford's Solid Tumor Actionable Mutation Panel

275 (STAMP) ${ }^{52}$. Activation of the RAS and PI3K pathways were rarely explained by mutations in

276 NF 1, PTEN, or other genes profiled by STAMP (Table S5 and S6), likely due to the

277 noncomprehensive tumor suppressor gene panel characterized by STAMP, as well as epigenetic

278 mechanisms of RAS and PI3K pathway activation. Epigenetic silencing and other non-genomic

279 mechanisms have been well documented to inhibit tumor suppressor genes including PTEN ${ }^{22,23,}$

28053,54 . Therefore, we performed immunohistochemistry for PTEN on 20 oncogene-negative lung

281 adenocarcinomas that did not have genomic PTEN mutations. Consistent with previous reports,

282 we observed low PTEN protein levels in 13 out of 20 of these tumors (Figure S14a-f) ${ }^{22}$.

283 To assess a larger set of oncogene-negative lung adenocarcinomas for alterations that

284 could lead to the activation of RAS and PI3K pathways, we analyzed oncogene-negative tumors

285 in TCGA and GENIE datasets. We queried a set of well-established negative regulators of the

286 RAS and PI3K pathways for alterations in oncogene-negative tumors (Table S6). Consistent 
with previous reports, $N F 1$ and $R A S A 1$ alterations were enriched in oncogene-negative tumors;

288 however, coincident genomic alterations in NF1, RASA1, and PTEN were rare (Figure S14g-h)

$28955,56$. However, over $60 \%$ of oncogene-negative lung adenocarcinomas in TCGA had alterations

290 in either the RAS or PI3K pathways, and $22 \%$ of these tumors had alterations in components of

291 both pathways, likely representing oncogene-negative $\mathrm{RAS}^{\mathrm{P} I 3 \mathrm{~K}}$ lung adenocarcinomas (Figure 4i).

292 These frequencies were lower in the GENIE dataset, possibly because only a fraction of the

293 known genes in these pathways were analyzed (Figure S14i). These histological and genomic

294 analyses support a model in which activation of the RAS and PI3K pathways in Onc-

295 negative $\mathrm{R}^{\mathrm{RAS} / \mathrm{PI} 3 \mathrm{~K}}$ tumors can be generated by diverse genomic and/or epigenetic alterations.

296 Finally, we assessed whether Onc-negative $\mathrm{R}^{\mathrm{RAS} / \mathrm{PI} 3 \mathrm{~K}}$ tumors in our mouse model more

297 broadly exhibit transcriptional features that are consistent oncogene-negative human lung

298 adenocarcinoma. We generated a gene expression signature of murine Onc-negative $\mathrm{e}^{\mathrm{RAS} / \mathrm{PI} 3 \mathrm{~K}}$

299 tumors comprised of genes that are higher in Nf1/Rasa1/Pten tumors relative to Kras tumors in

300 mice. We then calculated gene signature activity scores for each TCGA lung adenocarcinoma for

301 this Onc-negative ${ }^{\text {RAS/PI3K }}$ gene expression signature using single-sample GSEA (Table S4).

302 Interestingly, the Onc-negative $\mathrm{RAS} / \mathrm{PI} 3 \mathrm{~K}$ signature was highest in oncogene-negative human lung

303 adenocarcinomas relative to lung adenocarcinomas driven by oncogenic KRAS or other known

304 oncogenes (Figure $\mathbf{4 j}$ ). Collectively, these data indicate that the molecular and biochemical state

305 of mouse Onc-negative $\mathrm{RAS} / \mathrm{PI} 3 \mathrm{~K}_{\text {tumors recapitulates that of a substantial fraction of oncogene- }}$

306 negative human lung adenocarcinomas.

307

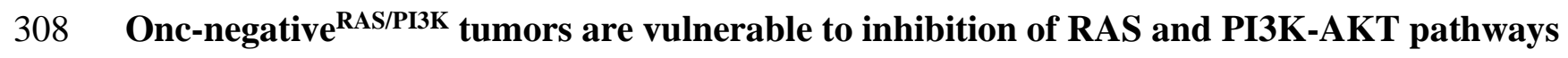


310 potential therapeutic strategies ${ }^{38}$. To investigate the therapeutic benefit of targeting key nodes in

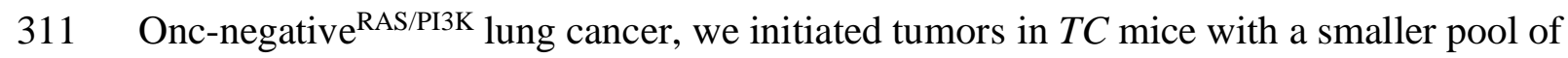

312 barcoded sgRNA viral vectors targeting Nfl, Rasal, and Pten. We treated these mice with the

313 SHP2 inhibitor RMC-4550 57 , AKT1/2 inhibitor capivasertib ${ }^{58,59}$, or a combination of the two

314 (Figure 5a and S15a-b). These drugs were chosen based on their ongoing clinical development 315 and ability to reduce activation RAS and PI3K pathways ${ }^{57,59 .}$

316 Direct fluorescence imaging and histology indicated that SHP2 inhibition and combined

317 SHP2 and AKT1/2 inhibition greatly reduced tumor burden (Figure 5b-c and S15c). Tuba-seq

318 analysis provided greater insights into the overall and genotype-specific responses of tumors to

319 the therapeutic interventions. Capivasertib monotherapy was ineffective in vivo while RMC-

3204550 reduced the total tumor burden. The combination of RMC-4550 and capivasertib trended

321 towards being the most efficient therapeutic approach reducing tumor burden by $\sim 30 \%$ compared

322 with RMC-4550 alone (Figure 5d, S15d-g).

323 We confirmed the inhibition of RAS and PI3K pathways in oncogene-negative $\mathrm{e}^{\mathrm{RAS} / \mathrm{PI} 3 \mathrm{~K}}$

324 tumors in mice treated with RMC-4550 and capivasertib by immunohistochemistry (Figure

325 S15h). Furthermore, global gene expression analysis confirmed the downregulation of RAS and

326 PI3K-AKT gene expression signatures after coincident SHP2 and AKT1/2 inhibition (Figure

327 S16a-d). Treated tumors tended to have higher expression of an apoptosis gene expression

328 signature and lower expression of a G2/M gene expression signature, suggesting that this

329 combination treatment induces broad cellular changes in oncogene-negative tumors (Figure

330 S16e-f). 
332 Inhibition of SHP2 and AKT synergizes to reduce the growth of Onc-negative ${ }^{\text {RAS/PI3K }}$ lung

333 adenocarcinoma cell lines

334 To more extensively characterize the responses to SHP2 and AKT inhibition, we

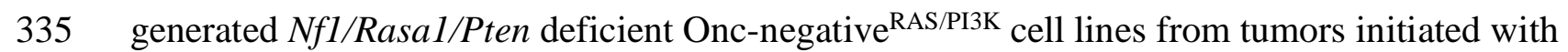

336 Lenti-sgNf1-sgRasal-sgPten/Cre in Trp53floxflox;TC mice (S17a-c). As anticipated, RAS and

337 PI3K signaling was reduced in response to treatment with RMC-4550 and capivasertib,

338 respectively (Figure S17d). RMC-4550 and capivasertib each decreased the overall growth of

339 three oncogene-negative $\mathrm{R}^{\mathrm{RAS} / \mathrm{PI} 3 \mathrm{~K}}$ cell lines in a dose-dependent manner (Figure 6a and S17e, g).

340 Consistent with our in vivo observations, RMC-4550 and capivasertib synergized to inhibit the

341 growth of these cell lines (Figure $\mathbf{6 b}$, and S17f, h). RAS and PI3K signaling can promote cell

342 growth and survival [58, 59], and RMC-4550 and capivasertib inhibited proliferation and

343 induced apoptosis to a greater extent than either RMC-4550 or capivasertib alone (Figure 6c-d).

344 Building on these findings, we assessed activation of RAS and PI3K pathways and driver

345 pathway vulnerabilities in two oncogene-negative human lung adenocarcinoma cell lines, NCI-

$346 \mathrm{H} 1838\left(N F 1^{\text {LOF }}\right)$ and NCI-H1623 (RASA $\left.1^{\text {LOF }}\right)$. H1838 and H1623 had activation of RAS and

347 PI3K pathways (Figure S17i). Consistent with our findings in mouse Onc-negative ${ }^{\text {RAS/PI3K }}$ cell

348 lines, RMC-4550 synergizes with capivasertib to inhibit the growth of these human Onc-

349 negative ${ }^{\text {RAS/PI3K }}$ lung adenocarcinoma cell lines (Figure 6e-f and S17j-k). These in vivo and cell

350 culture analyses indicate that Onc-negative ${ }^{\text {RAS/PI3K }}$ tumors are vulnerable to therapeutic inhibition

351 of these pathways.

352

353 DISCUSSION 
It is often overlooked that lung adenocarcinomas without genomic alterations in

355

356

357

358

359

360

361

362

363

364

365

366

367

368

369

370

371

372

373

374

375

376

oncogenes afflict as many patients as those driven by either oncogenic KRAS or EGFR. To

identify whether combinatorial inactivation of multiple tumor suppressor genes drives the

initiation and growth of lung adenocarcinoma in the absence of oncogene activation, we

performed a series of multiplexed in vivo functional genomic screens. By querying an extensive

set of tumor suppressor gene alterations, we uncovered combinatorial tumor suppressor

inactivation as a key driver of oncogene-negative lung adenocarcinomas. Importantly,

combinatorial inactivation of negative regulators of RAS and PI3K pathways are as potent as

oncogenic $\mathrm{KRAS}^{\mathrm{G} 12 \mathrm{D}}$ in initiating lung tumors in vivo.

Furthermore, while NF1 inactivation is sometimes suggested to be an "oncogenic driver"

in lung adenocarcinoma $4,29,60, N f 1$ inactivation alone is insufficient to initiate lung tumors

(Figure S8). Even pairwise inactivation of $N f 1$ and Rasal, as well as many other tumor

suppressor genes, generated very few tumors even after long time periods (Figure S8). These

data suggest that genomic and/or epigenetic alterations in multiple genes within and across

pathways may be required to surpass the thresholds necessary for Onc-negative ${ }^{\text {RAS/PI3K }}$ lung

adenocarcinoma initiation and growth.

Although cancers harbor diverse genomic and epigenomic alterations, these alterations

often converge on key pathways and generate similar biochemical changes ${ }^{15,61}$. For example,

myeloid leukemia can be driven by gain-of-function mutations in $K R A S, N R A S$, or the receptor

tyrosine kinase FLT3, or combined inactivation of multiple negative regulators of RAS pathway such as SPRY4 and NF1 ${ }^{62,63}$. Pathway activation through genomic and epigenomic inactivation of tumor suppressors can be very diverse, precluding the identification of non-oncogene drivers from gene-centric analysis of human cancer genomic data. Notably, our pathway analysis in 
377 oncogene-negative lung adenocarcinomas indicated that mutations in different genes that

378 converge on the RAS and PI3K pathways frequently co-occur (Figure 4i and S14i).

379 Furthermore, previous reports and our observations suggest frequent non-genomic mechanisms

380 of downregulation of RAS GAPs and PTEN (Figure $\mathbf{4 f - h , ~ S 1 4 a - f ) ~}{ }^{4,22-24,53,54}$. Thus, genomic

381 alterations should be viewed as a floor, not a ceiling, in estimating the frequency of pathway

382 alteration.

We assessed the ability of hundreds of complex tumor suppressor genotypes to generate

384 lung tumors. While activation of RAS and PI3K pathway emerged as the most potent driver of

385 oncogene-negative lung adenocarcinomas, our data also suggest that combinatorial inactivation

386 of tumor suppressor genes outside these two pathways can likely initiate tumorigenesis (Figure 2

387 and S6). Given the mutational diversity and complexity of oncogene-negative human lung

388 adenocarcinomas ${ }^{64}$, there remain many other mutational combinations to be investigated. We

389 anticipate that additional studies will uncover other oncogene negative tumor subtypes beyond

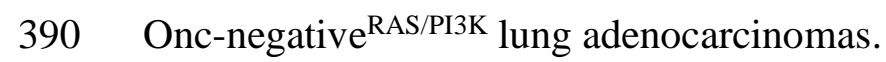

$391 \quad$ Knowledge of the genes underlying human cancer is a pillar of cancer diagnostics,

392 personalized medicine, and the selection of rational combination therapies. Additionally, our data

393 demonstrate RAS and PI3K pathway activation in the absence of oncogene mutations in a sizable

394 fraction of human lung adenocarcinoma that could predict therapeutic vulnerability to SHP2 and

395 AKT inhibitors. Thus, biochemical assessment of oncogenic pathways in tumors is a strong

396 foundation for rational selection of therapies and clinical trial designs. Beyond SHP2 and AKT,

397 extensive efforts have generated inhibitors for many other components of the RAS and PI3K

398 pathways. Thus, further investigation of the therapeutic targeting of key nodes within the RAS 
pathway (e.g., SOS, MEK, ERK) and PI3K pathway (e.g., PI3K, mTOR), could contribute to the development of the most effective therapies for Onc-negative $\mathrm{RAS}^{\mathrm{RA} I 3 \mathrm{~K}}$ lung adenocarcinomas. negative lung adenocarcinomas. This work identifies biomarkers and new therapeutic targets for

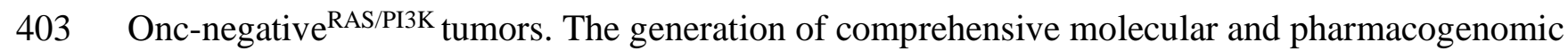

404 maps of oncogene-negative lung adenocarcinomas will transform our understanding of these 405 heretofore poorly characterized lung cancer subtypes.

\section{ACKNOWLEDGEMENTS}

We thank the Stanford Shared FACS Facility for flow cytometry and cell sorting

409 services, the Stanford Veterinary Animal Care Staff for expert animal care, Human

410 Pathology/Histology Service Center, Stanford Protein and Nucleic Acid Facility; A. Orantes and

411 S. Mello for administrative support; Stanford's Molecular Genetic Pathology Laboratory and

412 Henning Stehr for their help in providing genetically profiled tumor tissues. David Feldser,

413 Joseph Lipsick, Eric Collisson, Christopher McFarland, and members of the Winslow and Petrov

414 laboratories for helpful discussions and reviewing the manuscript. We thank Florent Elefteriou

415 and Alejandro Sweet-Cordero for providing mouse strains. M.Y. was supported by a Stanford

416 University School of Medicine Dean’s fellowship, an American Lung Association senior

417 research training grant, and an NIH Ruth L. Kirschstein National Research Service Award (F32-

418 CA236311). G.B., H.C., and J.D.H. were supported by a Tobacco-Related Disease Research

419 Program (TRDRP) Postdoctoral Fellowships (T31FT-1772, 28FT-0019, and T31FT-1619).

420 C.W.M. was supported by the NSF Graduate Research Fellowship Program and an Anne T. and

421 Robert M. Bass Stanford Graduate Fellowship. W-Y.L. was supported by an American 
Association of Cancer Research Postdoctoral fellowship (17-40-18-LIN). C.L. was the Connie

424 and C.I.C were supported by PHS Grant Number CA09302, awarded by the National Cancer

425 Institute, DHHS. E.L.A. was also supported by HHMI Gilliam Fellowship for Advanced Study

426 (GT14928). This work was supported by NIH R01-CA231253 (to M.M.W and D.A.P), NIH

427 R01-CA230919 (to M.M.W.) and NIH R01-CA234349 (to M.M.W and D.A.P.), as well as by

428 the Stanford Cancer Institute, an NCI-designated Comprehensive Cancer Center.

\section{CONFLICT OF INTERESTS}

431 S.K.C. receives grant support from Ono Pharma. C.S. acknowledges grant support from Pfizer,

432 AstraZeneca, Bristol Myers Squibb, Roche-Ventana, Boehringer-Ingelheim, Archer Dx, and Ono

433 Pharmaceuticals. C.S is an AstraZeneca Advisory Board member and Chief Investigator for the

434 MeRmaiD1 clinical trial, has consulted for Pfizer, Novartis, GlaxoSmithKline, MSD, Bristol

435 Myers Squibb, Celgene, AstraZeneca, Illumina, Amgen, Genentech, Roche-Ventana, GRAIL,

436 Medicxi, Bicycle Therapeutics, and the Sarah Cannon Research Institute, has stock options in

437 Apogen Biotechnologies, Epic Bioscience, GRAIL, and has stock options and is co-founder of

438 Achilles Therapeutics. D.A.P. and M.M.W. are founders of, and hold equity in, D2G Oncology

439 Inc.

\section{REFERENCES}

442 1. Barta, J.A., Powell, C.A. \& Wisnivesky, J.P. Global Epidemiology of Lung Cancer. Ann $443 \quad$ Glob Health 85 (2019).

444 2. Devarakonda, S., Morgensztern, D. \& Govindan, R. Genomic alterations in lung

445 adenocarcinoma. Lancet Oncol 16, e342-351 (2015).

446 3. McDermott, U., Downing, J.R. \& Stratton, M.R. Genomics and the continuum of cancer 447 care. N Engl J Med 364, 340-350 (2011). 
448 4. Cancer Genome Atlas Research, N. Comprehensive molecular profiling of lung

449 adenocarcinoma. Nature 511, 543-550 (2014).

450 5. Carrot-Zhang, J. et al. Whole-genome characterization of lung adenocarcinomas lacking the RTK/RAS/RAF pathway. Cell Rep 34, 108707 (2021).

6. Campbell, J.D. et al. Distinct patterns of somatic genome alterations in lung adenocarcinomas and squamous cell carcinomas. Nat Genet 48, 607-616 (2016).

7. Lawrence, M.S. et al. Discovery and saturation analysis of cancer genes across 21 tumour types. Nature 505, 495-501 (2014).

8. Vaishnavi, A. et al. Oncogenic and drug-sensitive NTRK1 rearrangements in lung cancer. Nat Med 19, 1469-1472 (2013).

9. Jonna, S. et al. Detection of NRG1 Gene Fusions in Solid Tumors. Clin Cancer Res 25, 4966-4972 (2019).

10. Soda, M. et al. Identification of the transforming EML4-ALK fusion gene in non-small-

11. Takeuchi, K. et al. RET, ROS1 and ALK fusions in lung cancer. Nat Med 18, 378-381 (2012).

12. Izumi, H. et al. The CLIP1-LTK fusion is an oncogenic driver in non-small-cell lung cancer. Nature (2021).

13. Vogelstein, B. et al. Cancer genome landscapes. Science 339, 1546-1558 (2013).

14. Yaffe, M.B. The scientific drunk and the lamppost: massive sequencing efforts in cancer discovery and treatment. Sci Signal 6, pe13 (2013).

15. Sanchez-Vega, F. et al. Oncogenic Signaling Pathways in The Cancer Genome Atlas. Cell 173, 321-337 e310 (2018).

16. Krogan, N.J., Lippman, S., Agard, D.A., Ashworth, A. \& Ideker, T. The cancer cell map initiative: defining the hallmark networks of cancer. Mol Cell 58, 690-698 (2015).

17. George, J. et al. Comprehensive genomic profiles of small cell lung cancer. Nature 524, 47-53 (2015).

18. Gouyer, V. et al. Mechanism of retinoblastoma gene inactivation in the spectrum of neuroendocrine lung tumors. Am J Respir Cell Mol Biol 18, 188-196 (1998).

19. Sekido, Y., Fong, K.M. \& Minna, J.D. Molecular genetics of lung cancer. Annu Rev Med 54, 73-87 (2003).

20. Meuwissen, R. et al. Induction of small cell lung cancer by somatic inactivation of both Trp53 and Rb1 in a conditional mouse model. Cancer Cell 4, 181-189 (2003).

21. Govindan, R. et al. Genomic landscape of non-small cell lung cancer in smokers and never-smokers. Cell 150, 1121-1134 (2012).

22. Soria, J.C. et al. Lack of PTEN expression in non-small cell lung cancer could be related to promoter methylation. Clin Cancer Res 8, 1178-1184 (2002).

23. Kazanets, A., Shorstova, T., Hilmi, K., Marques, M. \& Witcher, M. Epigenetic silencing of tumor suppressor genes: Paradigms, puzzles, and potential. Biochim Biophys Acta 1865, 275-288 (2016).

24. Ding, L. et al. Somatic mutations affect key pathways in lung adenocarcinoma. Nature 455, 1069-1075 (2008).

25. Lee, J.S., Grisham, J.W. \& Thorgeirsson, S.S. Comparative functional genomics for identifying models of human cancer. Carcinogenesis 26, 1013-1020 (2005).

493 Its Data. Cell 173, 283-285 (2018). 
27. Consortium, A.P.G. AACR Project GENIE: Powering Precision Medicine through an International Consortium. Cancer Discov 7, 818-831 (2017).

28. Jorge, S.E., Kobayashi, S.S. \& Costa, D.B. Epidermal growth factor receptor (EGFR) mutations in lung cancer: preclinical and clinical data. Braz J Med Biol Res 47, 929-939 (2014).

29. Skoulidis, F. \& Heymach, J.V. Co-occurring genomic alterations in non-small-cell lung cancer biology and therapy. Nat Rev Cancer 19, 495-509 (2019).

30. Saito, M. et al. Gene aberrations for precision medicine against lung adenocarcinoma. Cancer Sci 107, 713-720 (2016).

31. Rogers, Z.N. et al. A quantitative and multiplexed approach to uncover the fitness landscape of tumor suppression in vivo. Nat Methods 14, 737-742 (2017).

32. Rogers, Z.N. et al. Mapping the in vivo fitness landscape of lung adenocarcinoma tumor suppression in mice. Nat Genet 50, 483-486 (2018).

33. Winters, I.P. et al. Multiplexed in vivo homology-directed repair and tumor barcoding enables parallel quantification of Kras variant oncogenicity. Nat Commun 8, 2053 (2017).

34. Winters, I.P., Murray, C.W. \& Winslow, M.M. Towards quantitative and multiplexed in vivo functional cancer genomics. Nat Rev Genet 19, 741-755 (2018).

35. Cai, H., et al. A functional taxonomy of tumor suppression in oncogenic KRAS-driven lung cancer. Under consideration (2021).

36. Madisen, L. et al. A robust and high-throughput Cre reporting and characterization system for the whole mouse brain. Nat Neurosci 13, 133-140 (2010).

37. Chiou, S.H. et al. Pancreatic cancer modeling using retrograde viral vector delivery and in vivo CRISPR/Cas9-mediated somatic genome editing. Genes Dev 29, 1576-1585 (2015).

38. Lynch, T.J. et al. Activating mutations in the epidermal growth factor receptor underlying responsiveness of non-small-cell lung cancer to gefitinib. $N$ Engl J Med 350, 2129-2139 (2004).

39. Ohashi, K. et al. Characteristics of lung cancers harboring NRAS mutations. Clin Cancer Res 19, 2584-2591 (2013).

40. Lin, Q. et al. The association between BRAF mutation class and clinical features in BRAF-mutant Chinese non-small cell lung cancer patients. J Transl Med 17, 298 (2019).

41. Jackson, E.L. et al. Analysis of lung tumor initiation and progression using conditional expression of oncogenic K-ras. Genes Dev 15, 3243-3248 (2001).

42. Paez, J.G. et al. EGFR mutations in lung cancer: correlation with clinical response to gefitinib therapy. Science 304, 1497-1500 (2004).

43. Politi, K. et al. Lung adenocarcinomas induced in mice by mutant EGF receptors found in human lung cancers respond to a tyrosine kinase inhibitor or to down-regulation of the receptors. Genes Dev 20, 1496-1510 (2006).

44. Li, D. et al. Bronchial and peripheral murine lung carcinomas induced by T790M-L858R mutant EGFR respond to HKI-272 and rapamycin combination therapy. Cancer Cell 12, 81-93 (2007).

45. van Veen, J.E. et al. Mutationally-activated PI3'-kinase-alpha promotes de-differentiation of lung tumors initiated by the BRAF(V600E) oncoprotein kinase. Elife 8 (2019).

46. Dankort, D. et al. A new mouse model to explore the initiation, progression, and therapy of BRAFV600E-induced lung tumors. Genes Dev 21, 379-384 (2007). 
47. McFadden, D.G. et al. Mutational landscape of EGFR-, MYC-, and Kras-driven genetically engineered mouse models of lung adenocarcinoma. Proc Natl Acad Sci US A 113, E6409-E6417 (2016).

48. Weinreich, D.M., Delaney, N.F., Depristo, M.A. \& Hartl, D.L. Darwinian evolution can follow only very few mutational paths to fitter proteins. Science 312, 111-114 (2006).

49. Winslow, M.M. et al. Suppression of lung adenocarcinoma progression by Nkx2-1. Nature 473, 101-104 (2011).

50. Sweet-Cordero, A. et al. An oncogenic KRAS2 expression signature identified by crossspecies gene-expression analysis. Nat Genet 37, 48-55 (2005).

51. Agarwal, A. et al. The AKT/I kappa B kinase pathway promotes angiogenic/metastatic gene expression in colorectal cancer by activating nuclear factor-kappa B and betacatenin. Oncogene 24, 1021-1031 (2005).

52. Yang, S.R. et al. Comprehensive Genomic Profiling of Malignant Effusions in Patients with Metastatic Lung Adenocarcinoma. J Mol Diagn 20, 184-194 (2018).

53. Maertens, O. \& Cichowski, K. An expanding role for RAS GTPase activating proteins (RAS GAPs) in cancer. Adv Biol Regul 55, 1-14 (2014).

54. Song, M.S., Salmena, L. \& Pandolfi, P.P. The functions and regulation of the PTEN tumour suppressor. Nat Rev Mol Cell Biol 13, 283-296 (2012).

55. Hayashi, T. et al. RASA1 and NF1 are Preferentially Co-Mutated and Define A Distinct Genetic Subset of Smoking-Associated Non-Small Cell Lung Carcinomas Sensitive to MEK Inhibition. Clin Cancer Res 24, 1436-1447 (2018).

56. Kitajima, S. \& Barbie, D.A. RASA1/NF1-Mutant Lung Cancer: Racing to the Clinic? Clin Cancer Res 24, 1243-1245 (2018).

57. Nichols, R.J. et al. RAS nucleotide cycling underlies the SHP2 phosphatase dependence of mutant BRAF-, NF1- and RAS-driven cancers. Nat Cell Biol 20, 1064-1073 (2018).

58. Middleton, G. et al. The National Lung Matrix Trial of personalized therapy in lung cancer. Nature 583, 807-812 (2020).

59. Davies, B.R. et al. Preclinical pharmacology of AZD5363, an inhibitor of AKT: pharmacodynamics, antitumor activity, and correlation of monotherapy activity with genetic background. Mol Cancer Ther 11, 873-887 (2012).

60. O'Neill, A.C., Jagannathan, J.P. \& Ramaiya, N.H. Evolving Cancer Classification in the Era of Personalized Medicine: A Primer for Radiologists. Korean J Radiol 18, 6-17 (2017).

61. Hanahan, D. \& Weinberg, R.A. Hallmarks of cancer: the next generation. Cell 144, 646674 (2011).

62. Zhao, Z. et al. Cooperative loss of RAS feedback regulation drives myeloid leukemogenesis. Nat Genet 47, 539-543 (2015).

63. Lock, R. \& Cichowski, K. Loss of negative regulators amplifies RAS signaling. Nat Genet 47, 426-427 (2015).

64. Lawrence, M.S. et al. Mutational heterogeneity in cancer and the search for new cancerassociated genes. Nature 499, 214-218 (2013). 
bioRxiv preprint doi: https://doi.org/10.1101/2021.10.20.464849; this version posted December 14, 2021. The copyright holder for this preprint (which was not certified by peer review) is the author/funder. All rights reserved. No reuse allowed without permission.

Yousefi, Boross et al.

a

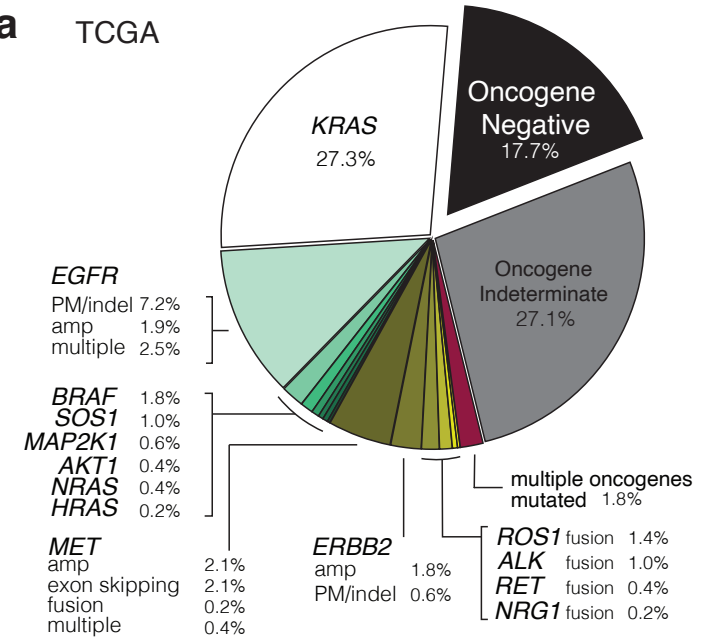

b

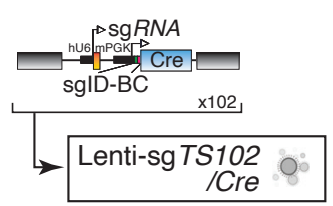

Arhgap35 Cmtr2 Lats1 Rb1

Arid1a Dnmt3a Lkb1 Rbm10

Arid1b Dot11 Lrp1b Rnf43

Arid2 Ep300 Mga Setd2

$\begin{array}{llll}\text { Atf7ip } & \text { Ercc4 Ncoa6 Smad4 } \\ \text { Atm } & \text { Fat1 } & \text { Ncor1 Smarca4 }\end{array}$

$\begin{array}{llll}\text { Atr } & \text { Fat1 } & \text { Ncor1 } & \text { Smarca4 } \\ \text { Atrx } & \text { Fbxw7 Nf1 } & \text { Stag2 }\end{array}$

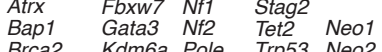

Brca2 Kdm6a Pole Trp53 Neo2

$\begin{array}{llll}\text { Cdkn2a } & \text { Keap1 Pten Tsc1 Neo3 } \\ \text { Cdkn2c } & \text { Kmt2c } & \text { Ptprd Ubr5 NT1 }\end{array}$

$\begin{array}{llll}\text { Cdkn2c } & \text { Kmt2c } & \text { Ptprd Ubr5 } & \text { NT1 } \\ \text { Cic } & \text { Kmt2d } & \text { Rasa1 Wrn NT2 }\end{array}$

2 sgRNÁs each sglnert

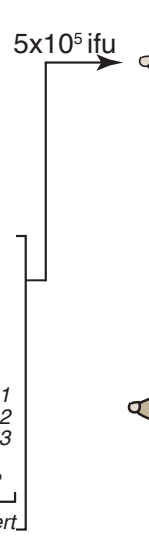

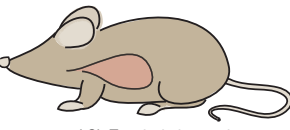

$R 26^{\text {LSL-Tom }}(T)(\mathrm{N}=9)$

$T ; H 11^{\text {LSL-Cas9 }}$ (TC) (N=7)

$N f 1^{t / f} ; T C(\mathrm{~N}=9)$

Pten $^{f / t} ; T C(\mathrm{~N}=3)$

$\operatorname{Trp53} 3^{f / f} ; T C(\mathrm{~N}=7)$

Lkb $1^{1 / f} ; T C(\mathrm{~N}=10)$
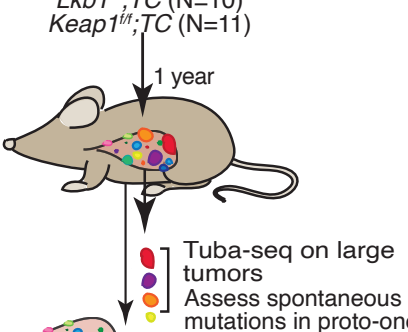

Tuba-seq on one lung lobe per mouse

C
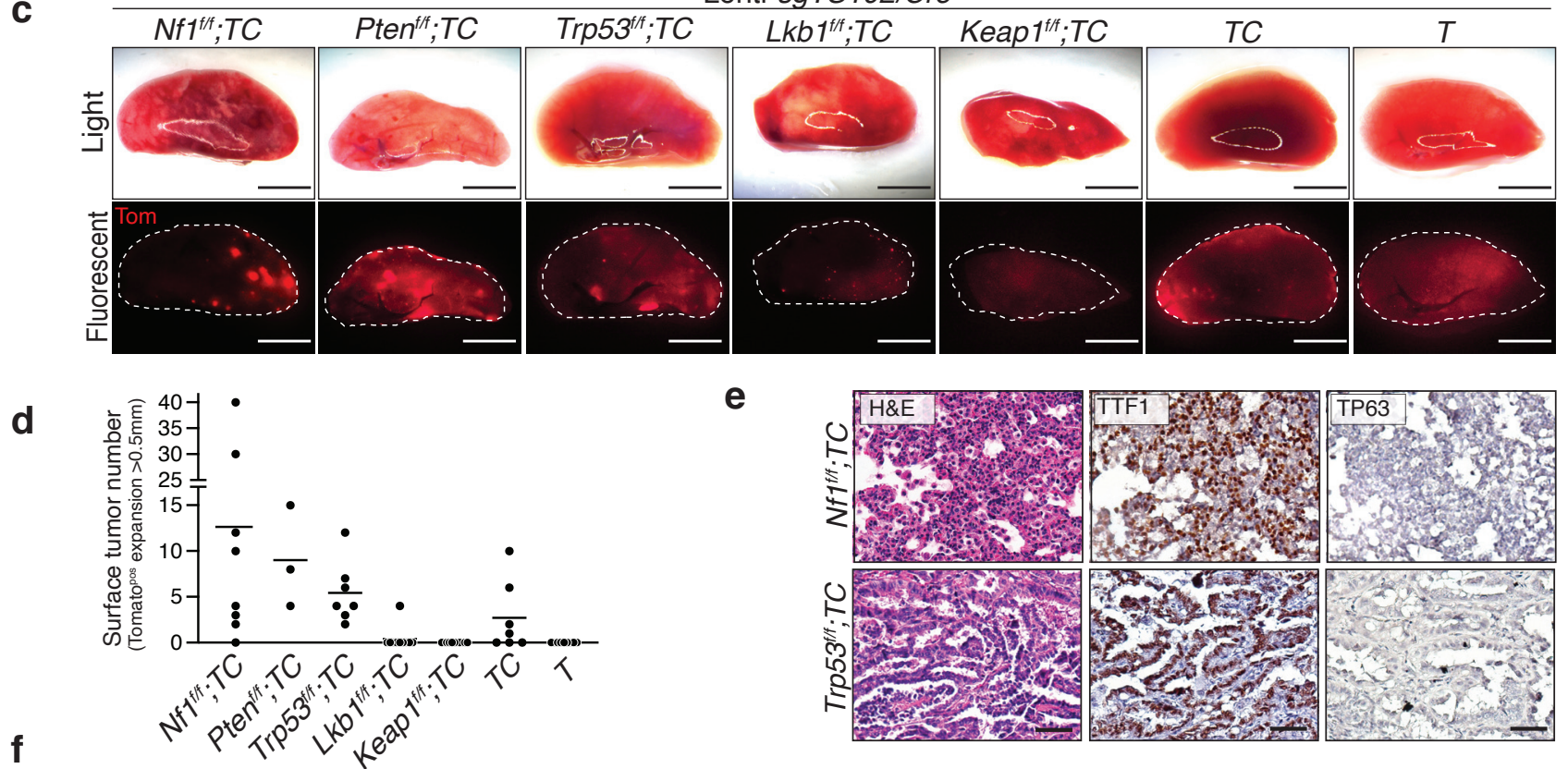

e
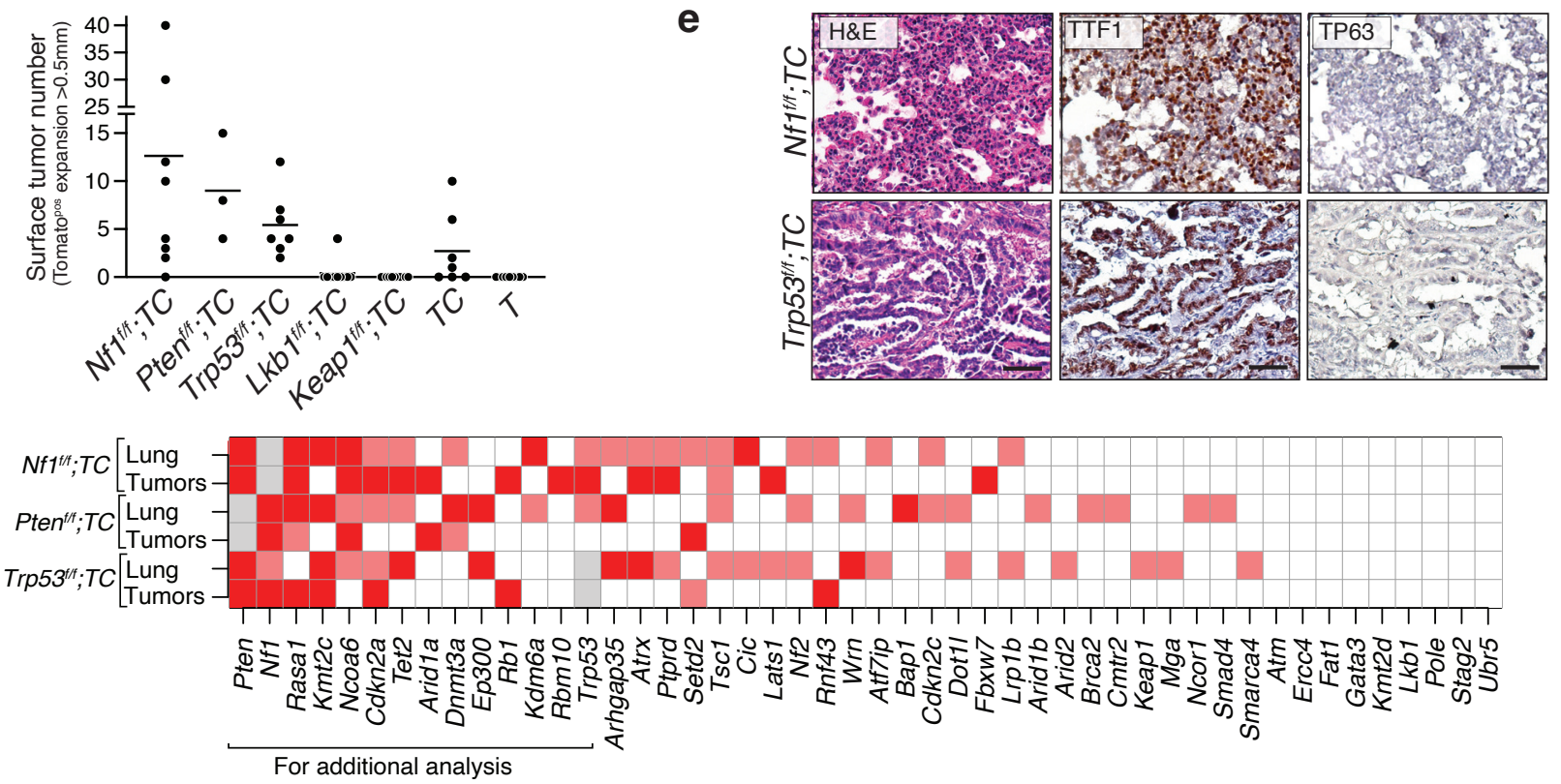

Figure 1. Combinatorial tumor suppressor inactivation enables lung tumor development in the absence of engineered oncogenes.

a. Frequency of human lung adenocarcinomas with likely oncogenic alterations in proto-oncogenes (oncogene-positive), with alterations of unknown effects in proto-oncogenes (oncogene-indeterminate), and without any alterations in proto-oncogenes (oncogene-negative). Data from TCGA. PM: point mutation, indel: insertion and deletion, amp: amplification, multiple: multiple alterations in the same gene.

b. Combined Cre/lox and CRISPR/Cas9-mediated tumor suppressor gene inactivation to generate lung epithelial cells with diverse genotypes. The number of mice in each group is indicated.

c. Representative light and fluorescence images of lung lobes from the indicated genotypes of mice one year after transduction with the Lenti-sg TS102/Cre pool. Lung lobes are outlined with white dotted lines. Scale bar $=4 \mathrm{~mm}$

d. The number of surface tumors (defined as Tomato-positive expansions greater than $0.5 \mathrm{~mm}$ in diameter) quantified by direct counting. Each dot represents a mouse, and the bar is the mean.

e. Representative Hematoxylin and Eosin (H\&E), TTF1, and TP63 stained sections of the indicated genotypes of mice. Scale bar $=100 \mu \mathrm{m}$

f. Heatmap showing two measures of tumor suppressor strengths in each genotype detected using Tuba-seq analysis: (1) in rows labeled as "Tumors" we assessed the occurrence of tumor suppressor gene targeting vectors in dissected tumors. $p<0.001$ (red), $p<0.1$ (pink) (see Figure S4)

(2) In rows labeled as "Lung" we measured increases in median sizes of clonal expansions in the presence of indicated tumor suppressor alterations in bulk single lung lobe samples. Gene mutations showing significant increases $(p<0.05)$ in sizes of clonal expansions using all sgRNAs are shown in red, and those with only one significant sgRNA are shown in pink (see Figure S5). Gray boxes indicate redundant targeting of genes by both Cre/loxP and CRISPR/Cas9. 
bioRxiv preprint doi: https://doi.org/10.1101/2021.10.20.464849; this version posted December 14, 2021. The copyright holder for this preprint (which was not certified by peer review) is the author/funder. All rights reserved. No reuse allowed without permission.

Yousefi, Boross et al.

a

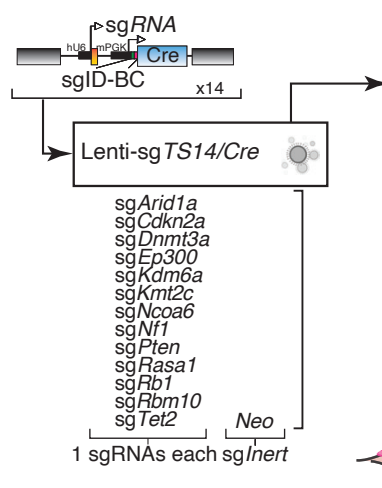

b

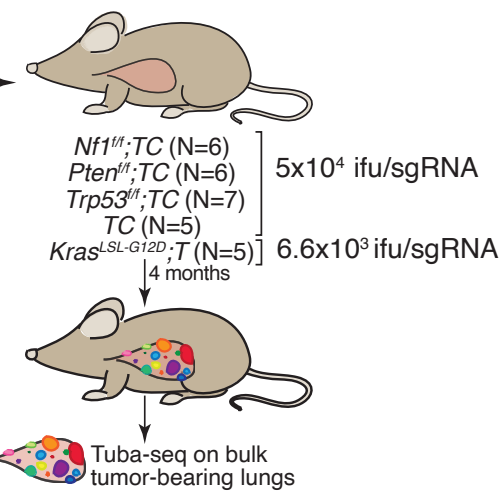

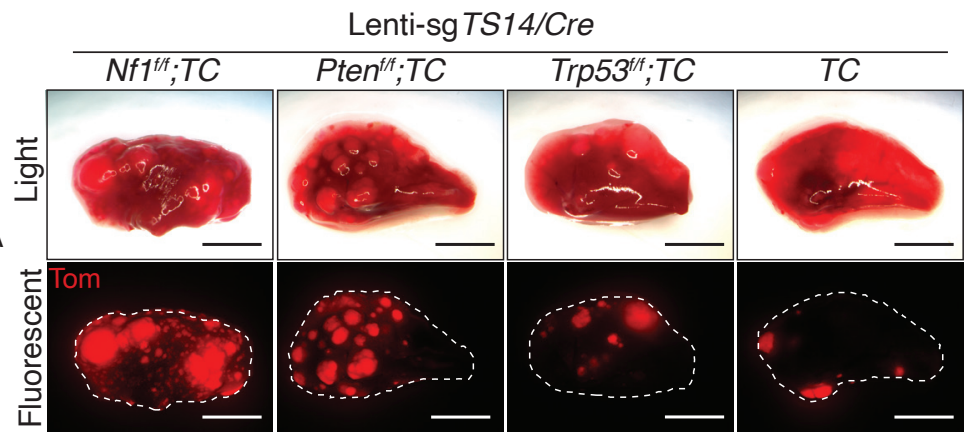

e

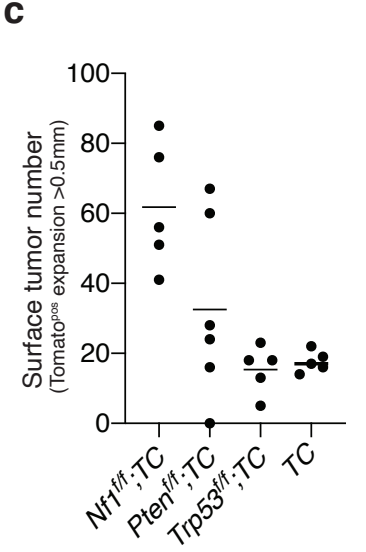

d

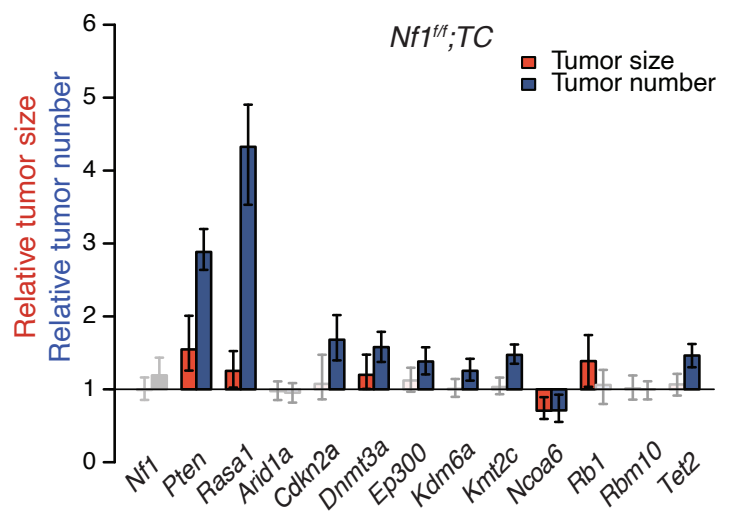

g

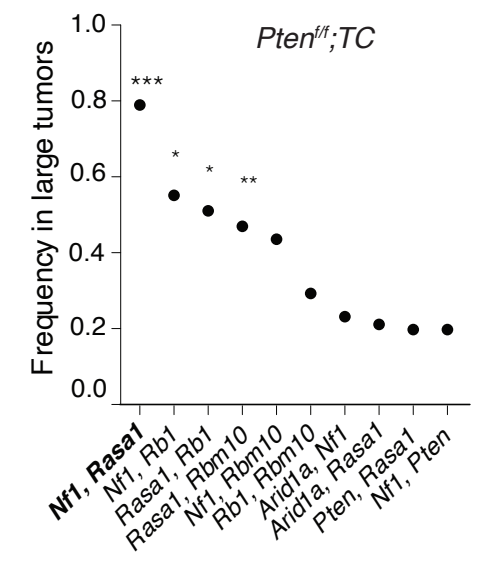

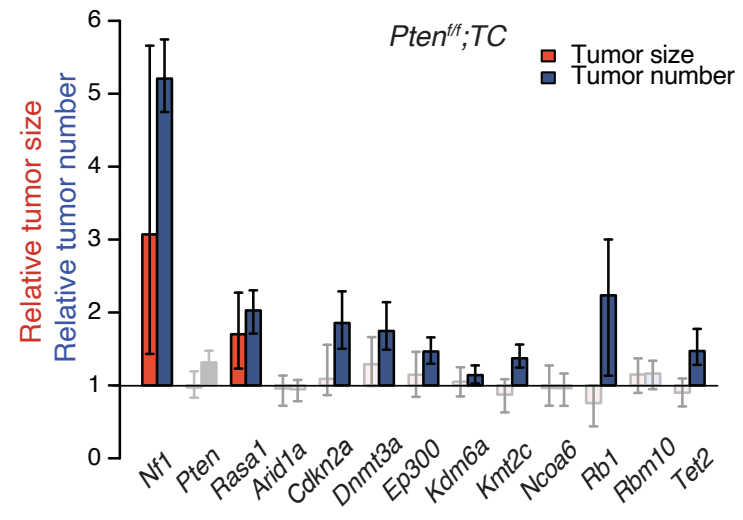

h
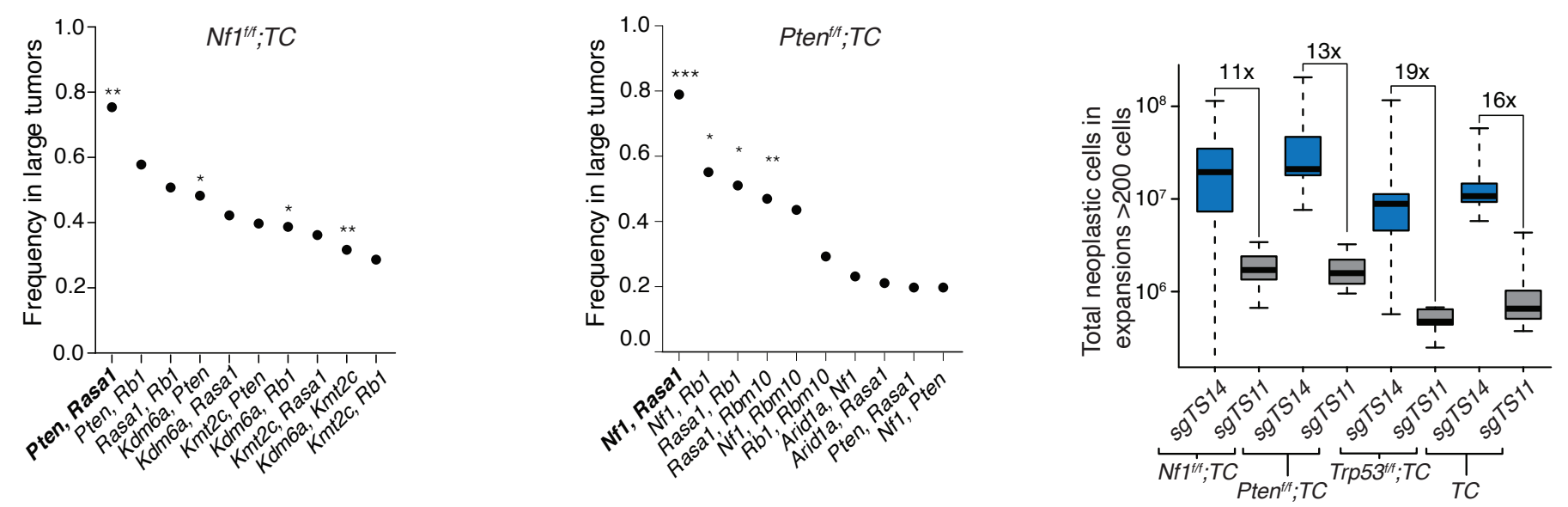

Figure 2. Nf1, Rasa1, and Pten emerge as key drivers of oncogene-negative lung adenocarcinoma.

a. Combined Cre/lox and CRISPR/Cas9-mediated tumor suppressor gene inactivation to generate lung epithelial cells with diverse genotypes. The number of mice in each group is indicated.

b. Representative light and fluorescence images of lung lobes from the indicated genotypes of mice. Lung lobes are outlined with white dotted lines. Scale bar $=4 \mathrm{~mm}$

c. The number of tumors (defined as Tomato-positive expnsions larger than $0.5 \mathrm{~mm}$ in diameter) quantified by direct counting. Each dot represents a mouse, and the bar is the mean.

d,e. The number of tumors with a minimum size of 1000 neoplastic cells relative to the inert sgRNA containing expansions is shown as blue bars. $90^{\text {th }}$ percentile of tumor sizes relative to the inert sgRNAs is shown as a red bar. sgRNAs resulting in significantly different tumor number or size ( $\mathrm{p}<0.05)$ are shown in darker colors. Whiskers show 95\% confidence intervals. Mouse genotypes are indicated.

f,g. Barcodes with the highest counts in each mouse were investigated for coinfection with multiple Lenti-sg TS/Cre vectors(i.e., tumors initiated from cells transduced with multiple viruses, which result in complex tumor suppressor genotypes, see Methods). The top 10 pairs of tumor suppressors that were most frequently co-mutated are shown. Combinations of sgRNAs that lead to the generation of Nf1, Rasa1, and Pten mutant cancer cells are in bold. ${ }^{*} p<0.05,{ }^{* *} p<0.01,{ }^{* * *} p<0.001$ based on a permutation test.

h. Total number of neoplastic cells in clonal expansions with more than 200 cells in the indicated genotypes of mice after receiving Lenti-sgTS14/Cre or Lenti-sgTS11/Cre, which lacks lentiviral vectors containing sgNf1, sgRasa1, and sgPten. The magnitude of neoplastic cell number reduction in each group is indicated. 
bioRxiv preprint doi: https://doi.org/10.1101/2021.10.20.464849; this version posted December 14, 2021. The copyright holder for this preprint (which was not certified by peer review) is the author/funder. All rights reserved. No reuse allowed without permission.

Yousefi, Boross et al.

a Lenti-sgTSTriple-pool/Cre

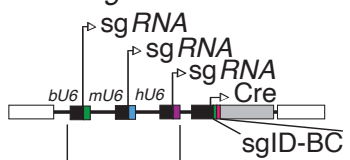

๘ $[\mathrm{sgNe01-sgNeo2-sgNT}$ [sgNf1-sgNeo2-sgNT ค. SgNeo1-sgRasa1-sgNT - sgNeo1-sgNeo2-sgPten o) [sgNf1-sgRasa1-sgNT $\stackrel{\leftarrow}{\sim}$ sgNf1-sgNeo2-sgPten of sgNeo1-sg Rasa1-sgPten $\stackrel{\circ}{\circ}$ [sgNf1-sgRasa1-sgPten

d
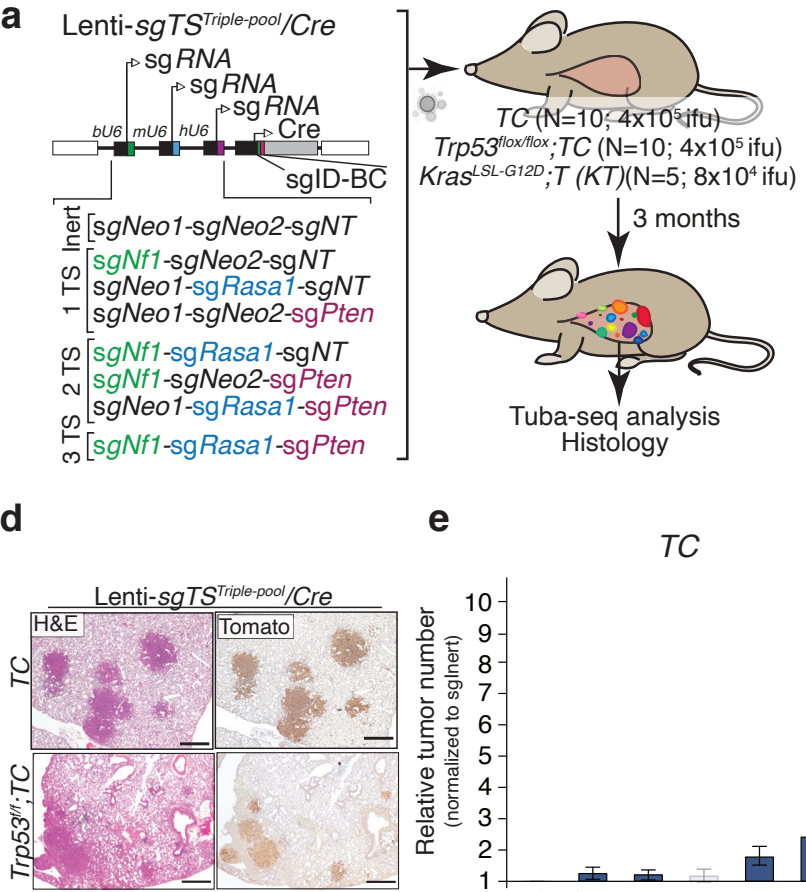

e

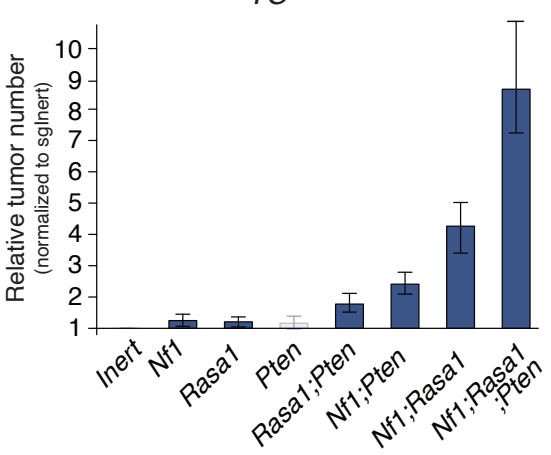

b

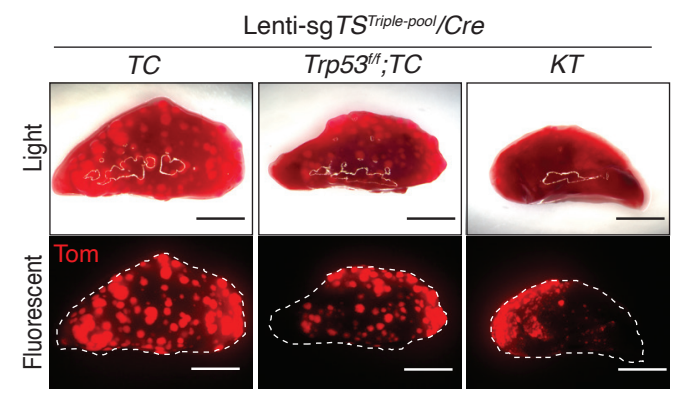

f

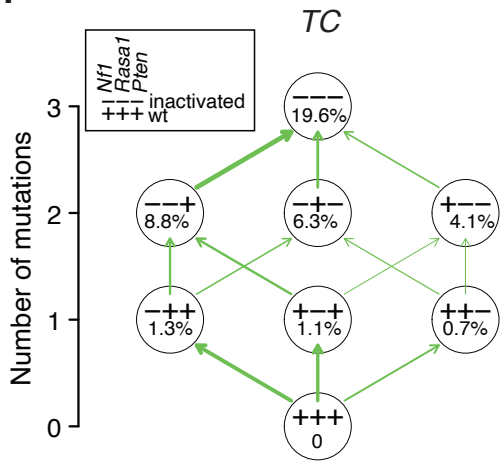

C

g
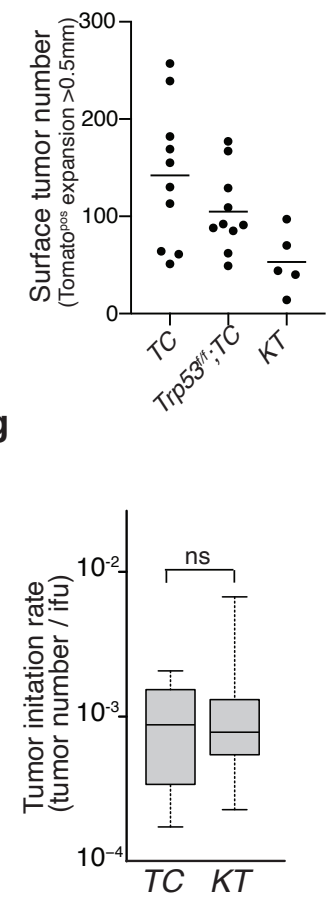

Figure 3. Inactivation of $\mathbf{N f 1}$, Rasa1, and Pten allows a stepwise acquisition of growth advantage during lung adenocarcinoma development. a. 8 barcoded triple sgRNA vectors for CRISPR/Cas9-mediated inactivation of all combinations of Nf1, Rasa1, and Pten in TC and Trp53 floxflox; TC mice to assess genetic interactions between these tumor suppressors. sgNeo1 and sgNeo2 are active cutting, but inert sgRNAs that target $\mathrm{NeO}^{R}$ in the $R 26^{L S L \text {-tdTomato }}$ allele. sgNT is a non-targeting inert sgRNA. Mouse genotype, mouse number, and titer of virus delivered to each mouse are indicated. Tuba-seq was performed on tumor-bearing lungs 3 months after tumor initiation.

b. Bright-field and fluorescence images of lungs from the indicated mouse genotypes. Lung lobes are outlined with a dashed white line. Scale bar $=4 \mathrm{~mm}$ c. The number of surface tumors (defined as Tomato-positive expansions larger than $0.5 \mathrm{~mm}$ in diameter) quantified by direct counting. Each dot represents a mouse, and the bar is the mean.

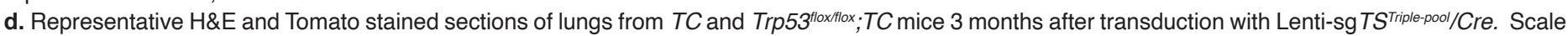
bar $=500 \mu \mathrm{m}$

e. Numbers of tumors (with $>1000$ neoplastic cells) relative to the Inert sgRNA containing expansions. sgRNAs resulting in a significantly higher number of tumors than the inert vector $(p<0.05)$ are shown in a darker color. Mean $+/-95 \%$ confidence interval is shown.

f. Adaptive landscape of Nf1, Rasa1, and Pten inactivation in TC mice is shown. Nodes represent genotypes shown as a string of + (wild-type) and - (inactivated) symbols representing Nf1, Rasa1, and Pten. Numbers in the nodes indicate fitness increase compared to wild-type. The relative probability of each beneficial mutation is shown as arrow widths (see Methods).

g. Quantification of the ability of combined Nf1/Rasa1/Pten inactivation in TC mice and oncogenic Kras ${ }^{G 12 D}$ in $K T$ mice to initiate tumors. The number of tumors (with $>1000$ neoplastic cells) per infectious unit (ifu) is shown. The bar is the median, the box represents the interquartile range, and the whiskers show minimum and maximum values. ns: non-significant 
bioRxiv preprint doi: https://doi.org/10.1101/2021.10.20.464849; this version posted December 14, 2021. The copyright holder for this preprint (which was not certified by peer review) is the author/funder. All rights reserved. No reuse allowed without permission.

Yousefi, Boross et al.

a

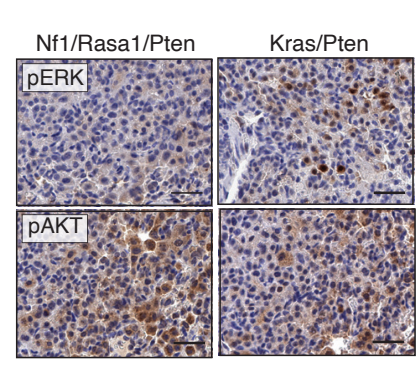

f

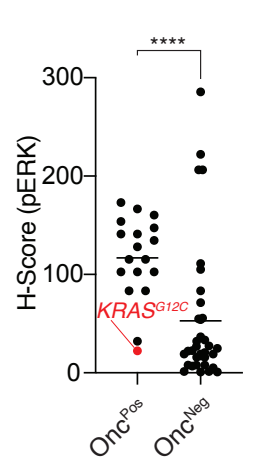

b

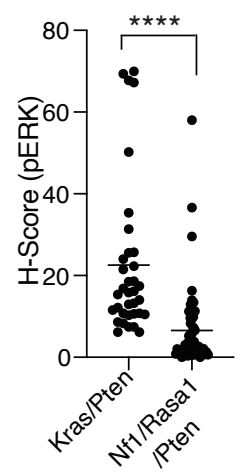

C

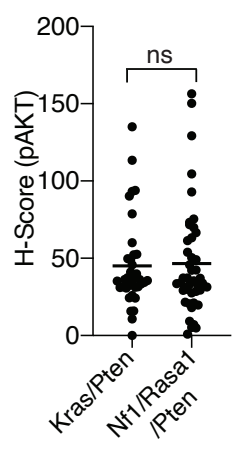

d

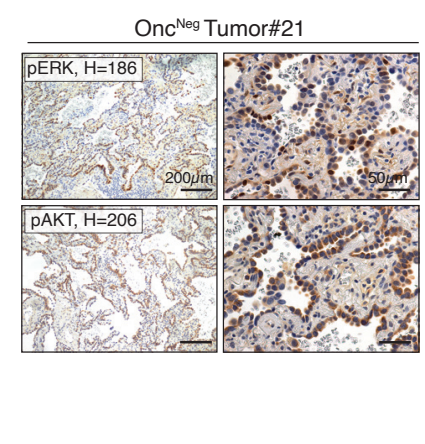

e

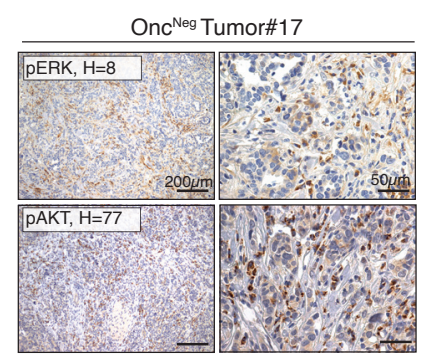

h

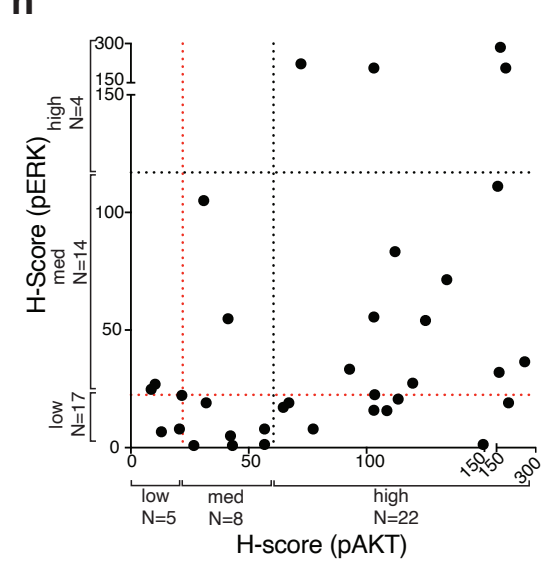

i

\section{TCGA}

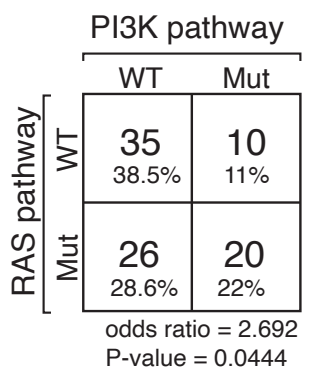

j

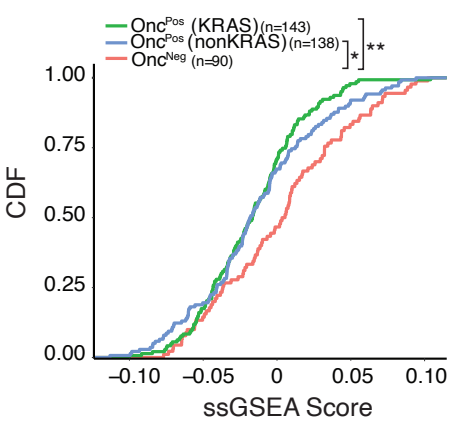

Figure 4. Oncogene-negative mouse and human lung adenocarcinomas have frequent activation of RAS and PI3K pathways.

a-c.Representative immunohistochemistry for pERK and PAKT to determine activation of RAS and PI3K pathway in tumors with the indicated genotypes and quantification of these stainings. The bar is the mean. n.s: non-significant, ${ }^{* * *}<\mathrm{p}<0.0001$ using Mann-Whitney U test. Scale bars $=50 \mu \mathrm{m}$

d,e. Representative p-AKT and p-ERK-stained sections of oncogene-negative human tumors. $\mathrm{H}$-scores for the whole section are indicated for each representative image. Scale bar $=200 \mu \mathrm{M}$ (right), $50 \mu \mathrm{m}$ (left)

f, g. Quantification of pAKT and pERK staining on 35 oncogene-negative and 18 oncogene-positive human lung adenocarcinomas. Genotypes of oncogenepositive tumors with the lowest pERK and pAKT staining intensities are highlighted in red. Significance between groups was determined using Mann-Whitney U test, ns: non-significant, ${ }^{* \star *} \mathrm{p}<0.0001$

h. pERK and pAKT H-scores for oncogene-negative human tumors are replotted from Figure 4f,g. Red dotted lines: the thresholds for low versus medium pERK and pAKT stains based on the lowest pERK staining intensity of oncogene-positive lung adenocarcinomas and the lowest pAKT staining level of EGFR mutant lung adenocarcinomas. Black dotted lines: the thresholds for medium versus high pERK and pAKT staining based on the mean pERK and pAKT H-scores in oncogene-positive tumors. The number of tumors in each staining intensity group (low, medium, high) is indicated on each axis of the plot.

i. Alteration frequency of well-established components of RAS and PI3K pathways (see Table S6) and assessment of their co-occurrences based on TCGA data sets. p-value calculated by two-sided Fisher's Exact Test.

j. Cumulative distribution function (CDF) plot of the signature scores for human tumors stratified by genes upregulated in mouse oncogene-negative tumors generated by inactivation of Nf1, Rasa1, and Pten (Figure S14a and see Table S4). The cohort size and the P-value calculated by Kolmogorov-Smirnov test are indicated on the plot. 
bioRxiv preprint doi: https://doi.org/10.1101/2021.10.20.464849; this version posted December 14, 2021. The copyright holder for this preprint (which was not certified by peer review) is the author/funder. All rights reserved. No reuse allowed without permission.

Yousefi, Boross et al.

a

Lenti-sgTripleTS6/Cre

sgNeo1-sgNeo2-sgNT sgNeo1-sgNeo2-sgPten sgNf1-sgRasa1-sgNT sgNf1-sgNeo2-sgPten sgNeo1-sgRasa1-sgPten sgNf1-sgRasa1-sgPten

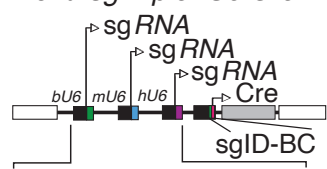

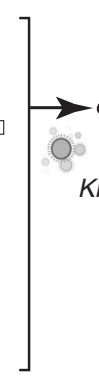

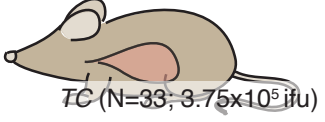

$K^{\prime a S}{ }^{L S L-G 12 D} ; T(K T)\left(\mathrm{N}=7 ; 9.37 \times 10^{4} \mathrm{ifu}\right)$

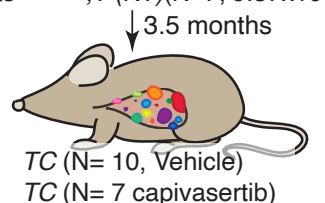

TC $(\mathrm{N}=7$ capivasertib)

TC $(\mathrm{N}=7$ RMC-4550)

TC $(\mathrm{N}=9$ RMC- 4550 \& capivasertib)

$\downarrow$ Drug treatment (2 weeks)

Tuba-seq \& Histology b

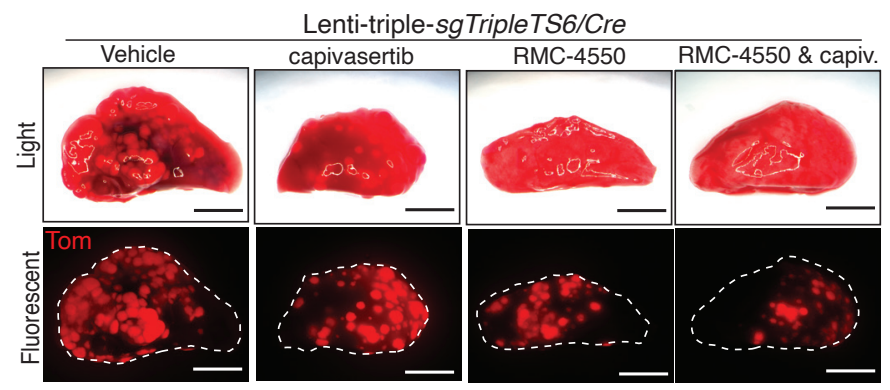

d

C

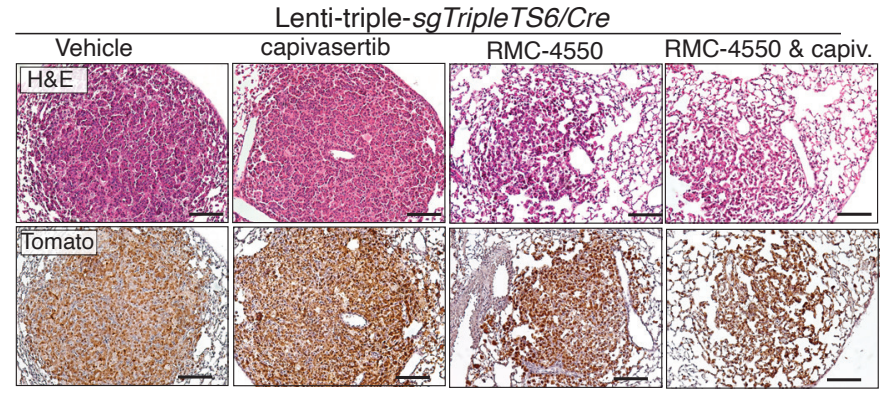

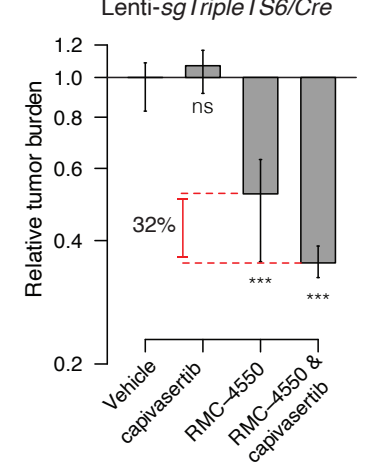

Figure 5. SHP2 and AKT inhibition synergize to reduce the growth of autochthonous oncogene-negative lung tumors.

a. Barcoded triple sgRNA vectors for CRISPR/Cas9-mediated inactivation of combinations of Nf1, Rasa1, and Pten in TC mice to determine the response of oncogene-negative tumors to pharmacological inhibition of RAS and PI3K pathways. Indicated numbers of mice were treated with RMC-4550 (SHP2 inhibitor), capivasertib (AKT inhibitor), or combination of these two drugs for two weeks 3.5 months after tumor initiation. Tuba-seq and histological analysis were performed on tumor-bearing lungs followed by analysis of tumor response to therapies.

b. Bright-field and fluorescence images of lungs from the indicated mice. Lung lobes are outlined with a dashed white line. Scale bars $=4$ $\mathrm{mm}$

c. Representative H\&E and Tomato-stained sections of tumors from TC mice 3.5 months after transduction with Lenti-sgTripleTS6/Cre and two weeks after treatment with the indicated drugs. Scale bars $=100 \mu \mathrm{m}$

d. Relative tumor burden in mice after treatment with capivasertib, RMC-4550, and combination of these two drugs compared with tumor burden in vehicle-treated mice. ns: non-significant, ${ }^{* \star *} p<0.001$. Drug response is shown for all the tumors. 
bioRxiv preprint doi: https://doi.org/10.1101/2021.10.20.464849; this version posted December 14, 2021. The copyright holder for this preprint (which was not certified by peer review) is the author/funder. All rights reserved. No reuse allowed without permission.

\section{Yousefi, Boross et al.}

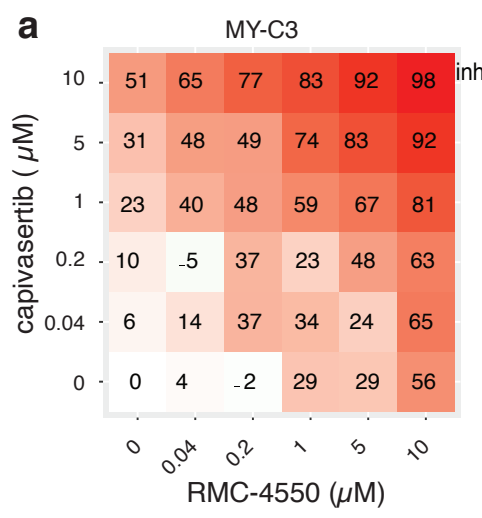

e

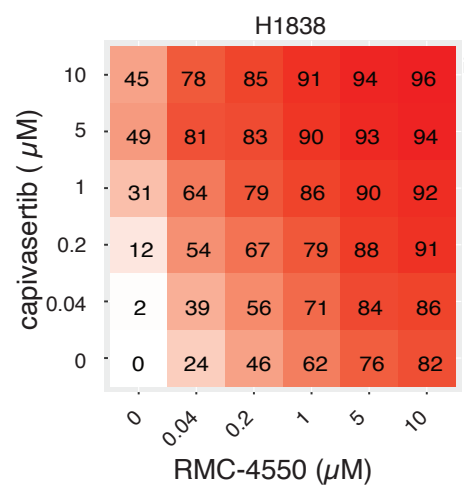

b

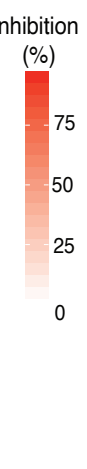

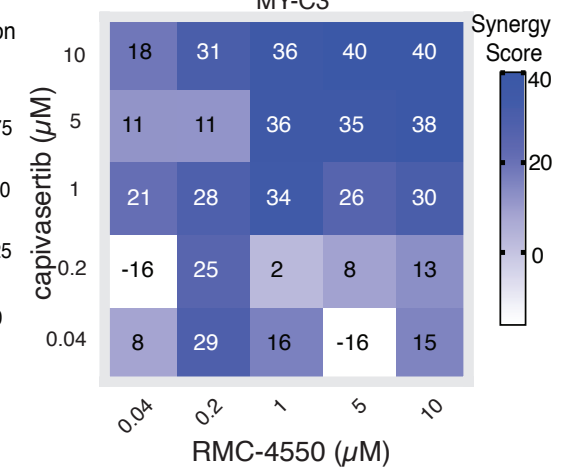

C

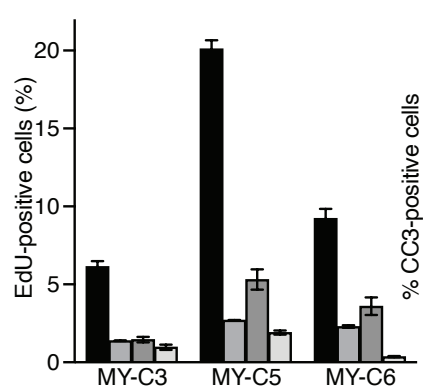

d

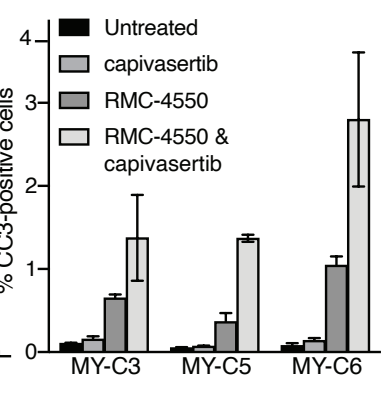

f

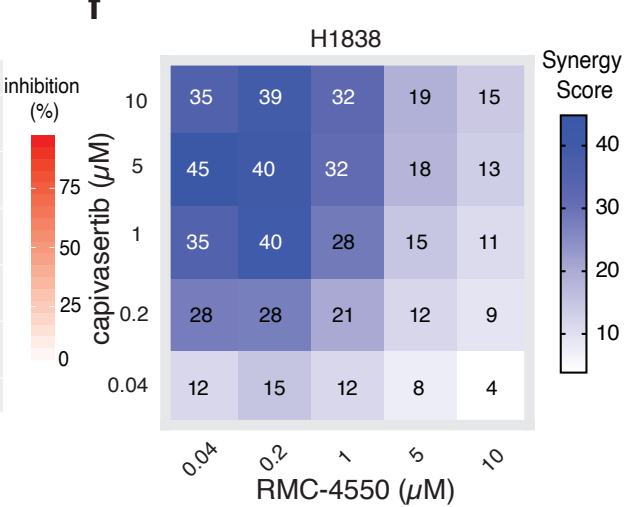

g

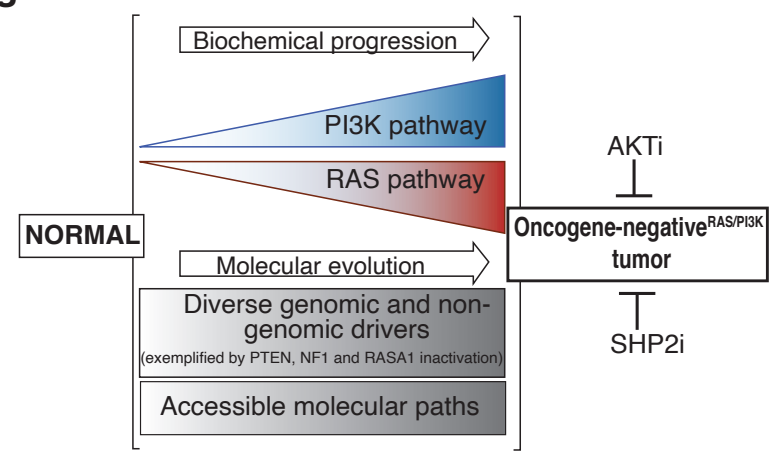

Figure 6. RMC-4550 and capivasertib synergize to inhibit the growth of Onc-negative ${ }^{\mathrm{RAS} / \mathrm{PI} K \mathrm{~K}}$ lung adenocarcinoma cell lines.

a. Drug dose-response matrix depicting \% growth inhibition of amurine Onc-negative ${ }^{\text {RAS/PI3K }}$ cell line after four days of treatment with the indicated doses of RMC-4550 and capivasertib. The average responses of three to four replicates are shown for each drug/drug combination. (see also Figure S17 a-h) b. Loewe's synergy score calculated based on drug responses in Figure 6a. Synergy scores indicate the percentage of response beyond expectation. c,d. Cell proliferation and apoptosis analysis using EdU incorporation, cleaved caspase 3 staining, and flow-cytometry analysis. Three independent Onc-negative ${ }^{\mathrm{RAS} / \mathrm{PI} 3 \mathrm{~K}}$ murine cell lines were treated with $10 \mu \mathrm{M}$ of the indicated drug/drugs for 2 days before the analysis.

e. Drug dose-response matrix depicting \% growth inhibition of $\mathrm{H} 1838$, a human oncogene-negative ${ }^{\text {RAS/PI3K }}$ lung adenocarcinoma cell line.

f. Loewe's synergy score calculated based on drug responses in Figure 6e.

g. Model of biochemical progression and molecular drivers of Onc-negative ${ }^{\text {RAS/PI3K }}$ tumors. 
bioRxiv preprint doi: https://doi.org/10.1101/2021.10.20.464849; this version posted December 14, 2021. The copyright holder for this preprint (which was not certified by peer review) is the author/funder. All rights reserved. No reuse allowed without permission.

Yousefi, Boross et al.
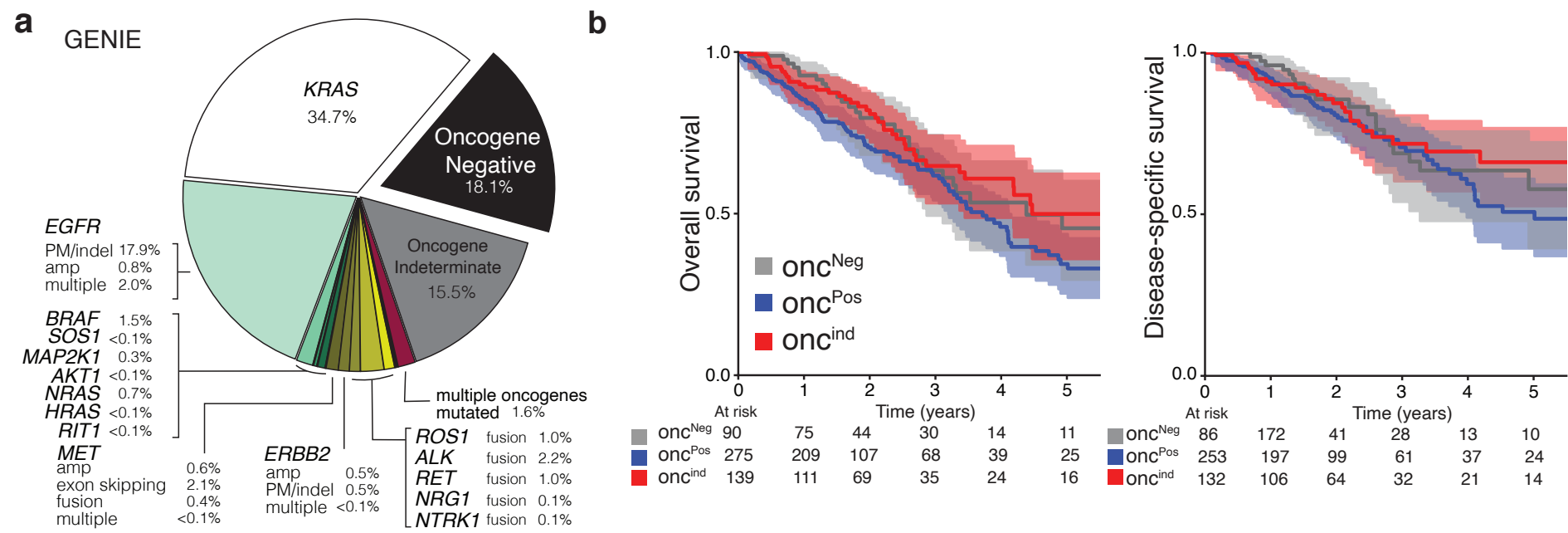

C

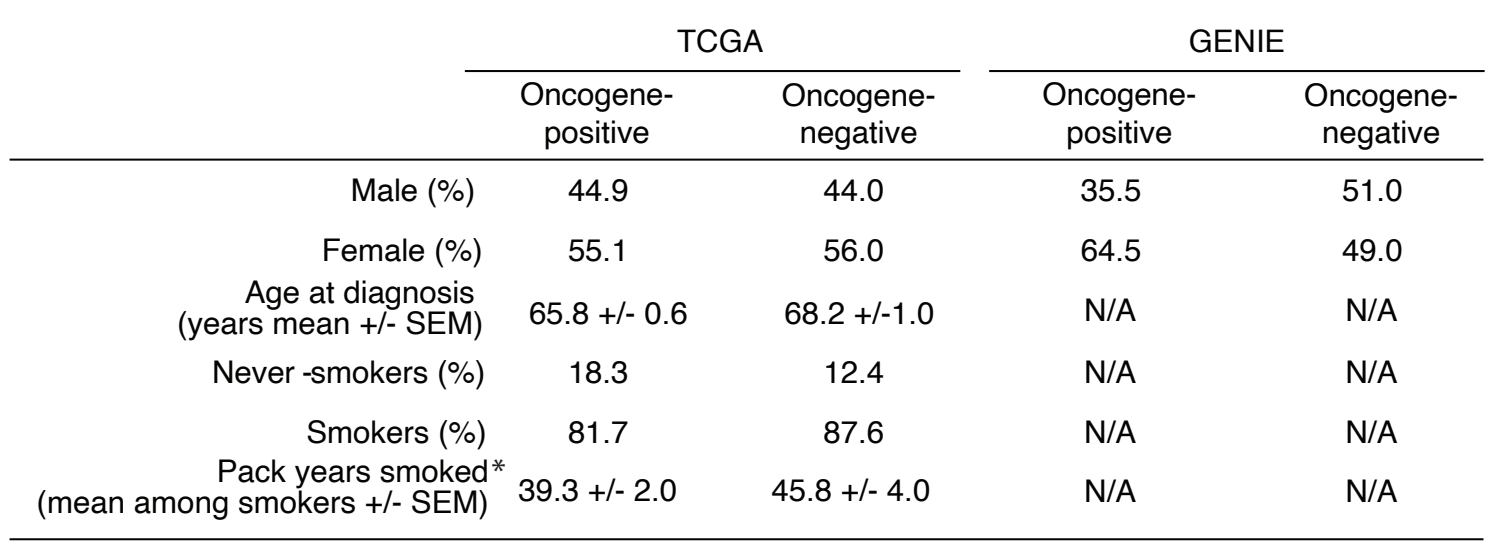

d

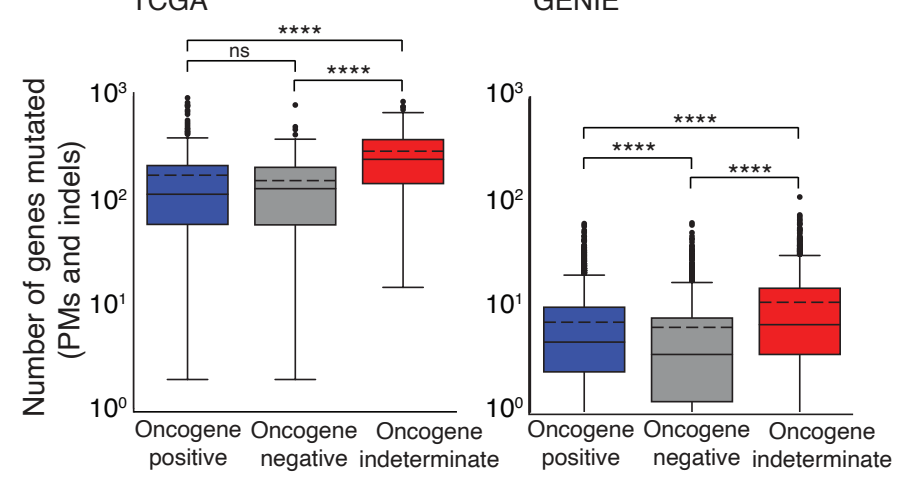

e

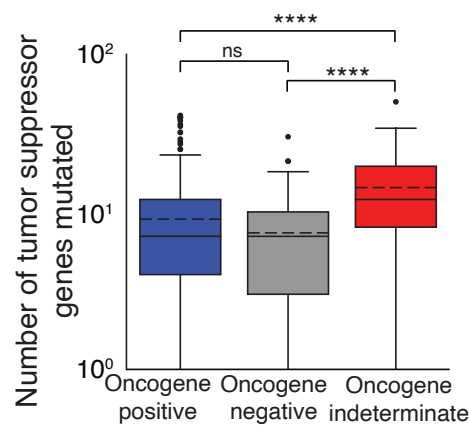

GENIE

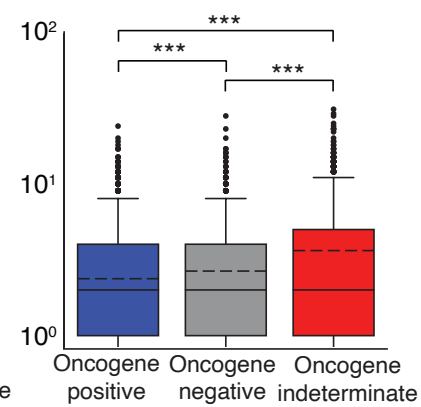

Figure S1. Clinical and molecular features of oncogene-negative human lung adenocarcinomas.

a. Frequency of human oncogene-positive, oncogene-indeterminate, and oncogene-negative lung adenocarcinomas based on GENIE data sets. PM: point mutation, indel: insertion and deletion, amp: amplification, multiple: multiple oncogenic alterations in the same gene (see Methods)

b. Overall survival and disease-specific survival of oncogene-positive (Onc $\left.{ }^{\mathrm{Pos}}\right)$, oncogene-indeterminate (Onc $\left.{ }^{\mathrm{lnd}}\right)$, and oncogene-negative $\left(\mathrm{Onc}^{\mathrm{Neg}}\right)$ lung adenocarcinoma patients based on TCGA data. The numbers below the plots are the numbers of patients alive at each time point.

c. Clinical characteristics of oncogene-positive and oncogene-negative patients based on TCGA and GENIE data sets. SEM standard error of the mean. N/A - information not present in this dataset. The $p$-values were calculated using Mann Whitney $U$ test, * $\mathrm{p}<0.05$.

d,e. The number of mutated genes (d,by point mutations (PMs) and indels) and number of mutated tumor suppressor genes (e, by point mutations, indels, or deletions) in oncogene-positive, oncogene-indeterminate, and oncogene-negative tumors based on TCGA and GENIE data sets. The mean is represented by the dashed line and the median by the straight line. ${ }^{\star \star \star \star} p<0.0001, p$-values were calculated using Mann Whitney U test. 
bioRxiv preprint doi: https://doi.org/10.1101/2021.10.20.464849; this version posted December 14, 2021. The copyright holder for this preprint (which was not certified by peer review) is the author/funder. All rights reserved. No reuse allowed without permission.

Yousefi, Boross et al.

a

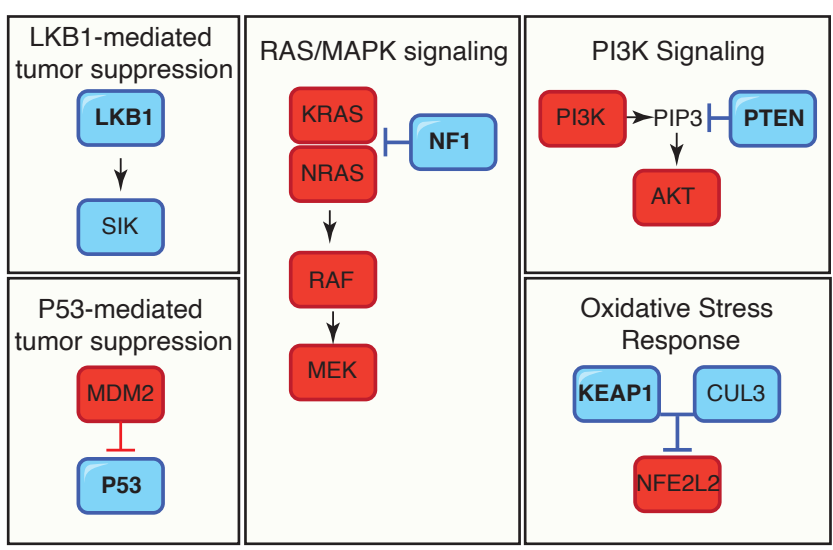

C

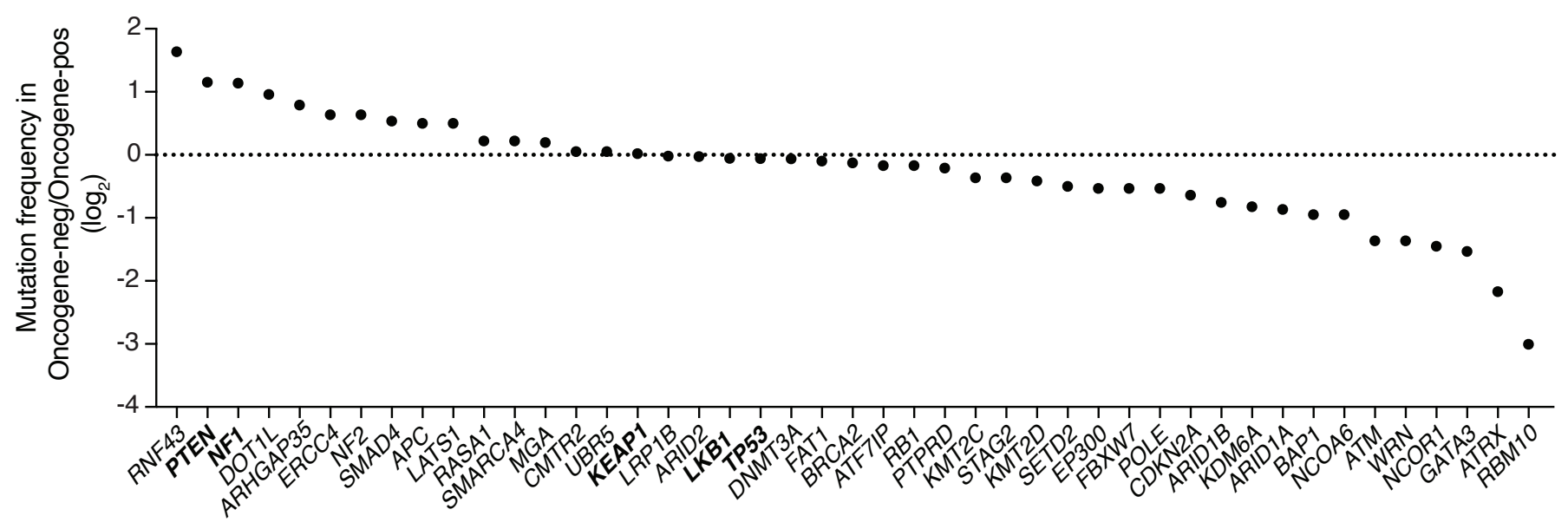

d

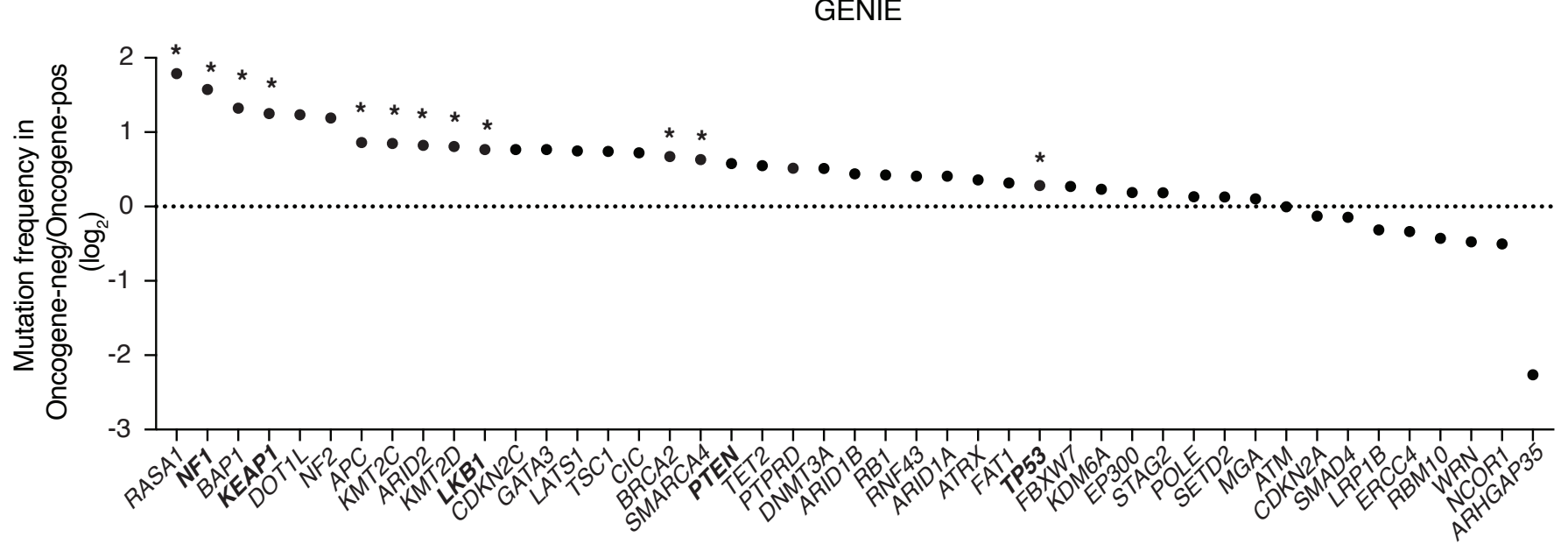

Figure S2. Tumor suppressor gene alterations in oncogene-negative lung adenocarcinoma.

a. Schematic of the pathways controlled by the five tumor suppressor genes inactivated using floxed alleles ("core" tumor suppressor genes) in this study. The tumor suppressors represent different key cancer pathways.

b. Alteration frequency of "core" tumor suppressor genes (number of tumors with potentially inactivating missense or nonsense mutations or focal DNA copy number losses/ total tumor number) in oncogene-negative lung adenocarcinomas based on GENIE and TCGA data sets.

c,d. The ratio of the frequency of inactivating alterations of tumor suppressor genes (point mutations, indel, and copy number loss) of the genes in the Lenti-sgTS102/Cre and Lenti-sgTS15/Cre pools in oncogene negative versus oncogene-positive lung adenocarcinomas. Data from TCGA (c) and GENIE (d) data sets are shown. The dotted line represents equal frequency in oncogene-negative and oncogene-positive lung adenocarcinomas. The "Core" tumor suppressors are in bold. *FDR $<0.05$ 
bioRxiv preprint doi: https://doi.org/10.1101/2021.10.20.464849; this version posted December 14, 2021. The copyright holder for this preprint (which was not certified by peer review) is the author/funder. All rights reserved. No reuse allowed without permission.

Yousefi, Boross et al.

a

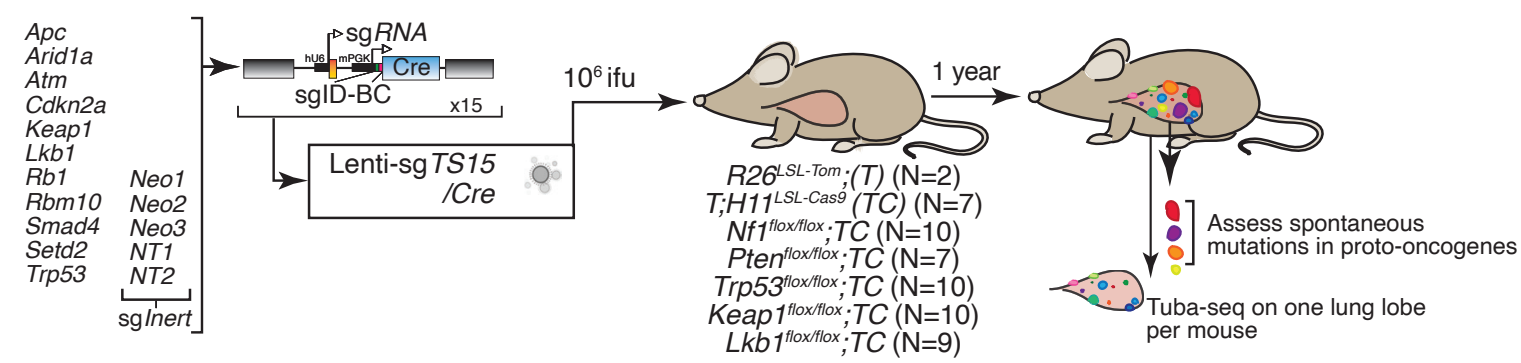

b

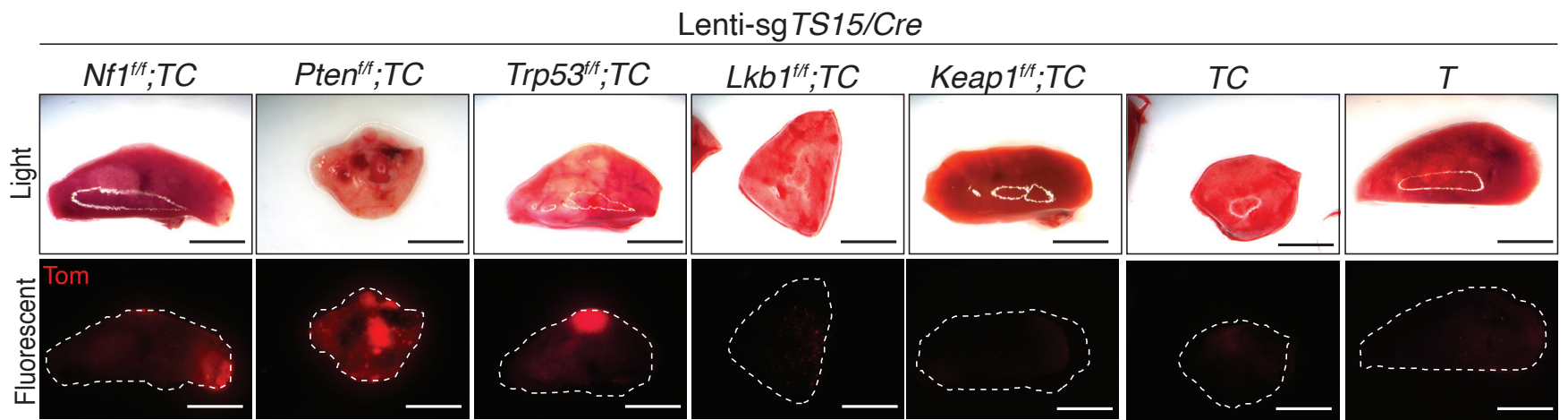

C

d

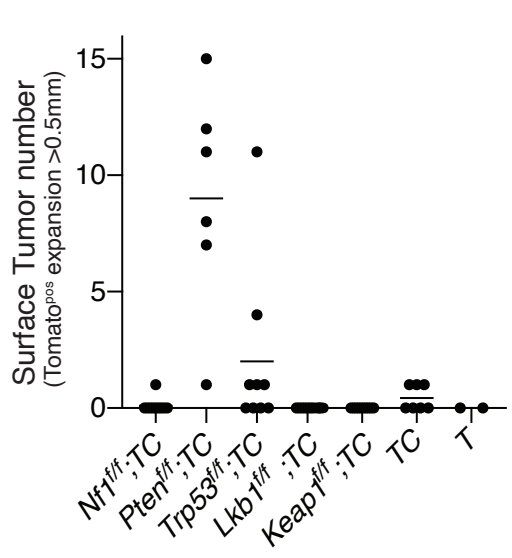

\begin{tabular}{ccccc} 
Gene & Exon & Key Codons & $\begin{array}{c}\text { Mutation } \\
\text { frequency }\end{array}$ & Mutations \\
\hline Kras & 2 & G12+G13 & $1 / 29$ & G12V \\
& 3 & Q61 & $0 / 29$ & none \\
Egfr & 18 & 721 & $0 / 29$ & none \\
& 19 & $734-756$ & $0 / 29$ & none \\
& 20 & $764-793$ & $0 / 29$ & none \\
& 21 & $860+863$ & $0 / 29$ & none \\
Braf & 14 & $503-509$ & $0 / 29$ & none \\
& 18 & V637(V600 equivalent) & $0 / 29$ & none \\
\multirow{5}{*}{ Nras } & 2 & G12+G13 & $0 / 29$ & none \\
& 3 & Q61 & $0 / 29$ & none \\
\hline
\end{tabular}

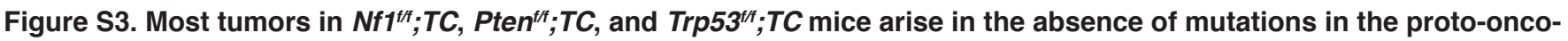
genes.

a. Schematic of combined Cre/lox and CRISPR/Cas9-mediated tumor suppressor gene inactivation to generate lung epithelial cells with diverse genotypes.

b. Representative light and fluorescence images of lung lobes from the indicated genotypes of mice. Lung lobes are outlined with white dotted lines. Scale bar $=4 \mathrm{~mm}$

c. The number of tumors (defined as Tomato-positive expansions greater than $0.5 \mathrm{~mm}$ in diameter) was quantified by direct counting. Each dot represents a mouse, and the bar is the mean.

d. Exons in known proto-oncogenes that were analyzed by targeted sequencing. Key codons are those in which mutations are associated with oncogenic activity. Mutation frequency is the number of tumors with putative oncogenic mutations over the total number of samples analyzed. Putative oncogenic mutations are defined in Methods. 
bioRxiv preprint doi: https://doi.org/10.1101/2021.10.20.464849; this version posted December 14, 2021. The copyright holder for this preprint (which was not certified by peer review) is the author/funder. All rights reserved. No reuse allowed without permission.

Yousefi, Boross et al.

a

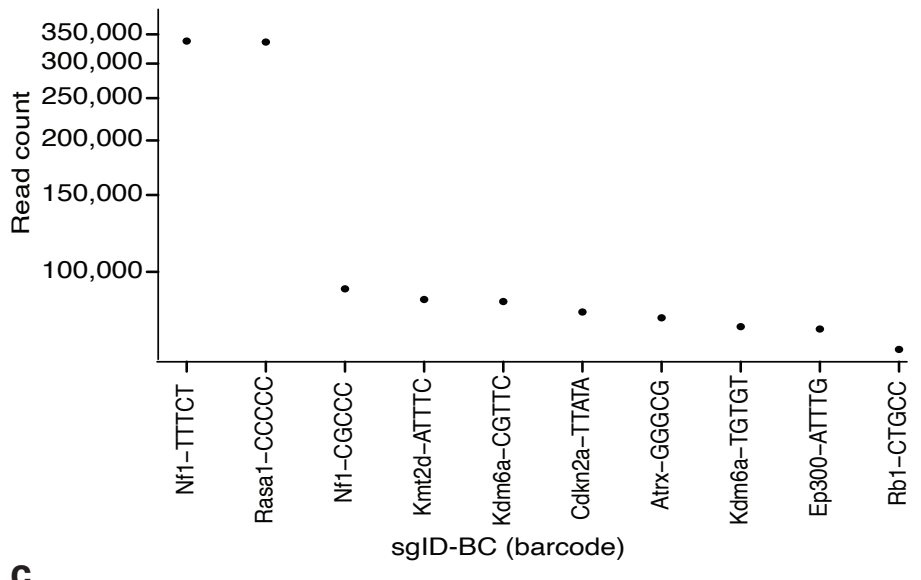

C

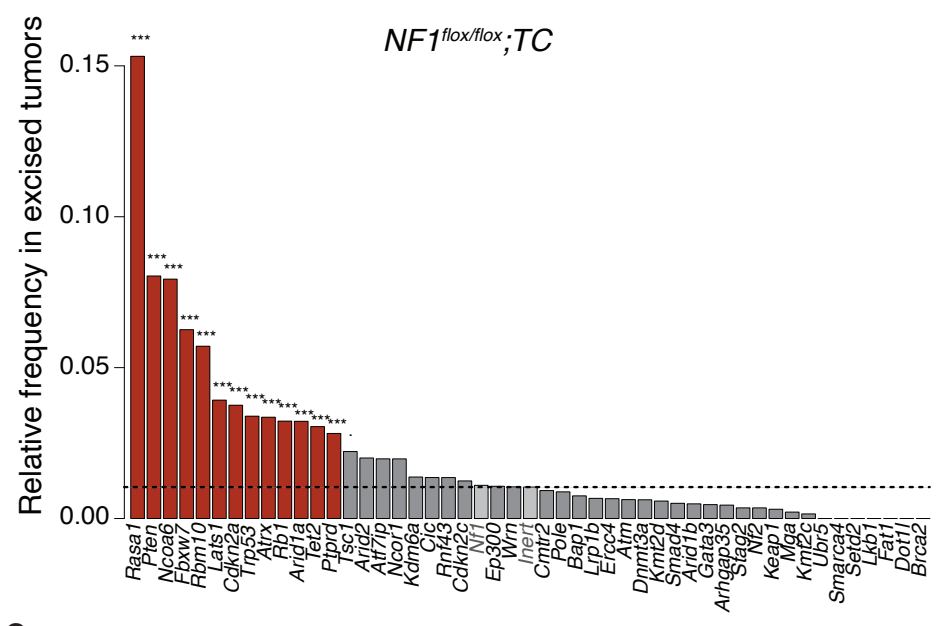

e

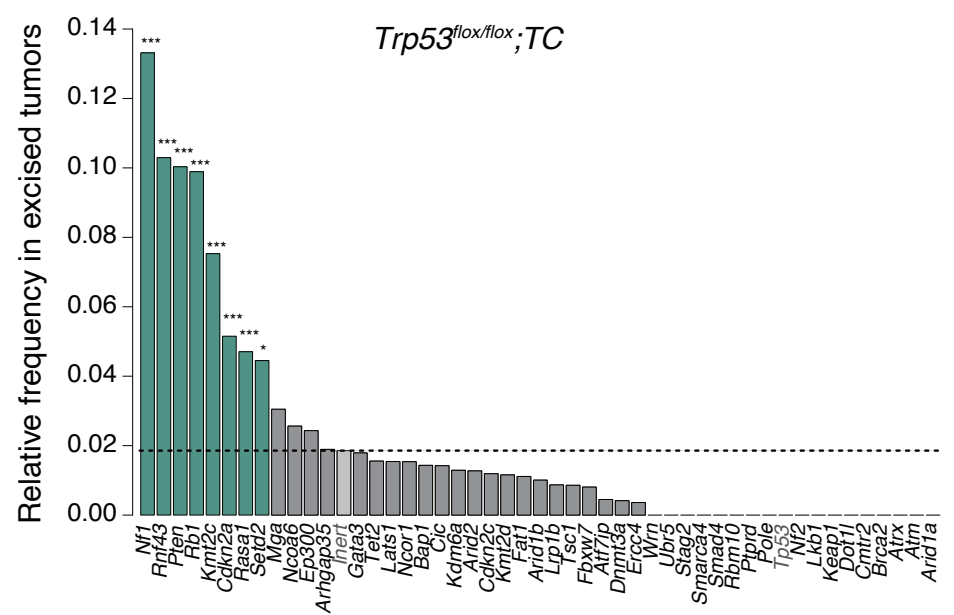

b

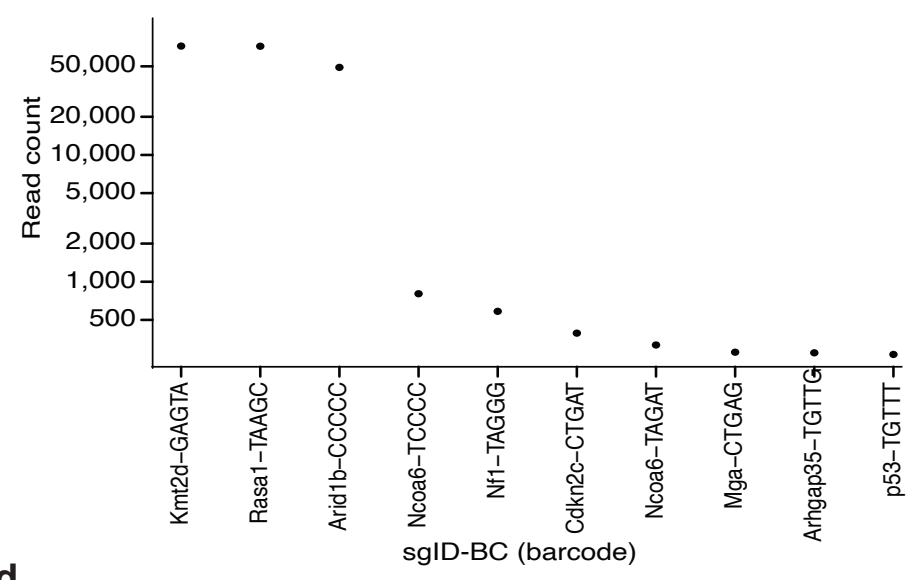

d
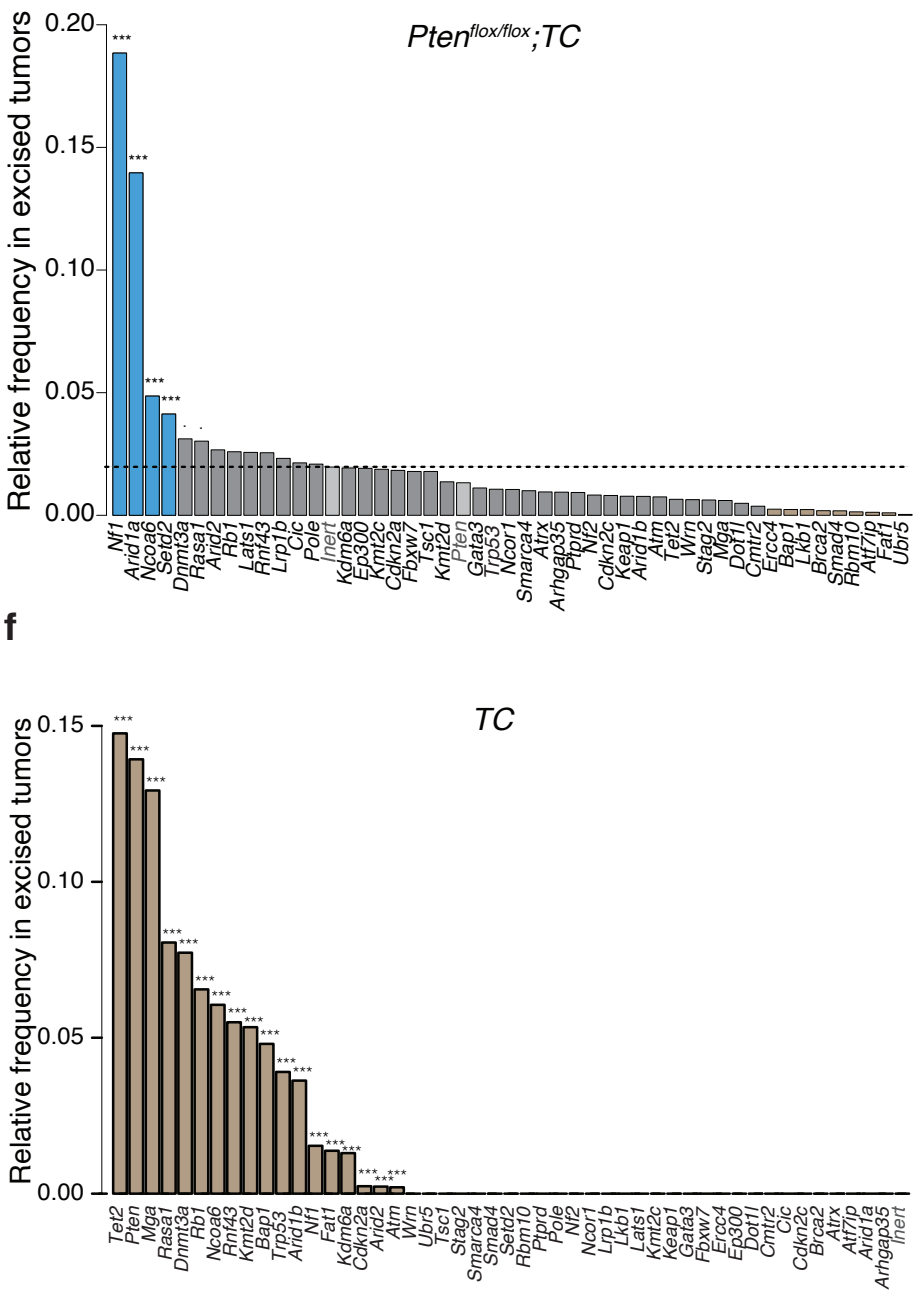

Figure S4. Identification of tumor suppressor genes that constrain lung tumor formation in vivo.

a,b. Example plots indicating evidence of transduction with multiple barcoded lentiviral vectors. The10 sgID-BCs with the highest read counts from two excised tumors are shown. Each dot represents a sgID-BC, the $y$-axis shows read count, and the sgID-BCs are sorted on the $x$-axis by decreasing read counts (the first 5 nucleotides of the random barcode are shown with the targeted gene symbol). The first two and three barcodes (sgID-BC) in subpanels a and b, respectively, that have very similar read counts likely represent a single clonal tumor initiated from a cell transduced with multiple barcoded Lenti-sgRNA/Cre vectors (see Methods Multiple transduction section).

c-f. Relative frequency of sgRNAs targeting each tumor suppressor gene in tumors harvested from the indicated genotypes of mice. Tumors were dissected under a fluorescence microscope based on their tdTomato fluorescent signal and were subjected to genomic DNA extraction. The sgID-BC region was PCR amplified and sequenced using Illumina high-throughput sequencing. The dotted lines represent the frequency of the inert sgRNA (average of all inert sgRNAs). Genes significantly overrepresented compared to the inert sgRNA are shown as: ** $p<0.001,{ }^{*} p<0.05, \cdot p<0.1$. Light gray bars indicate sgRNAs targeting the "core" tumor suppressor gene that is inactivated with floxed alleles in each plot and the inert sgRNAs. 
bioRxiv preprint doi: https://doi.org/10.1101/2021.10.20.464849; this version posted December 14, 2021. The copyright holder for this preprint (which was not certified by peer review) is the author/funder. All rights reserved. No reuse allowed without permission.

Yousefi, Boross et al.

a
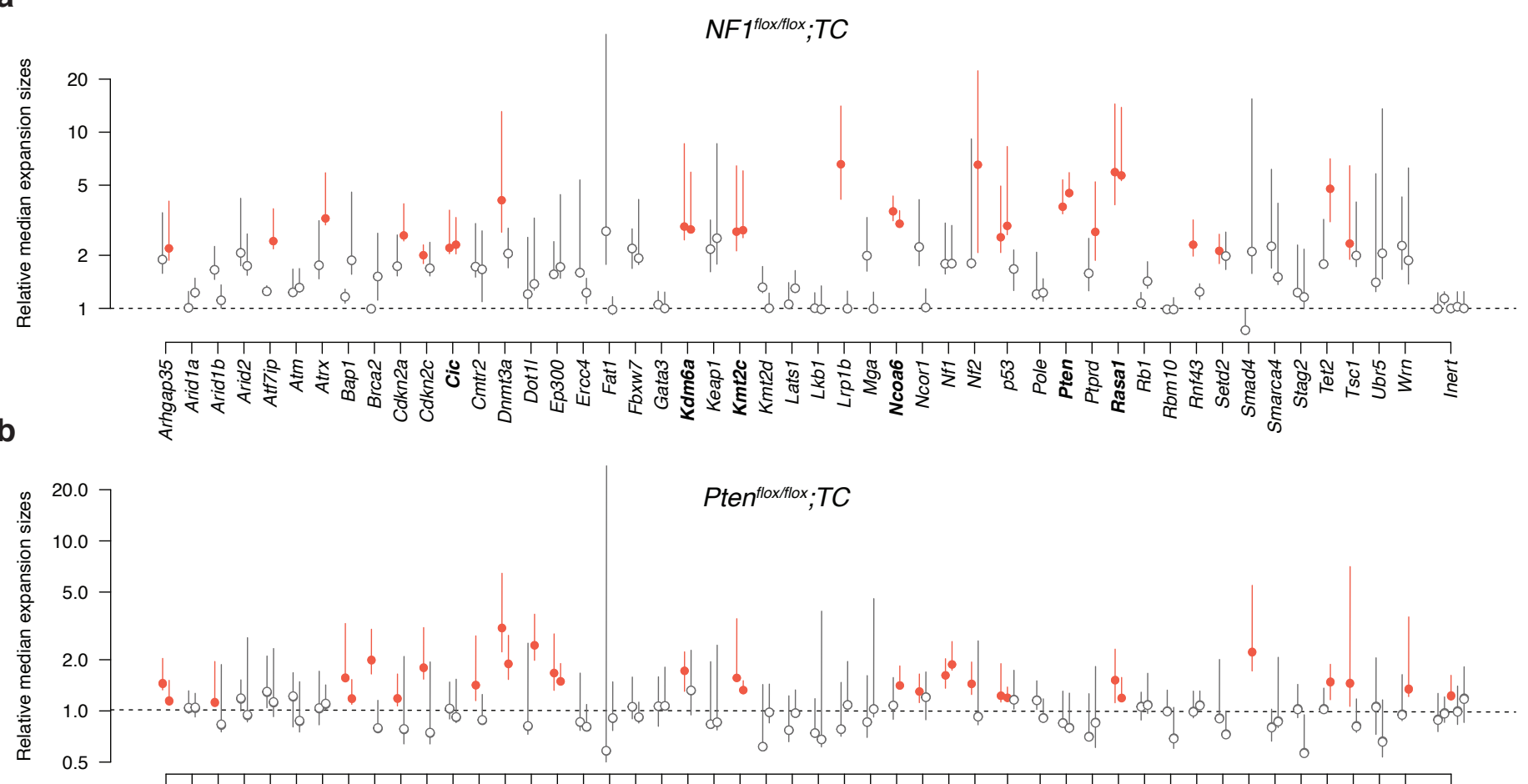

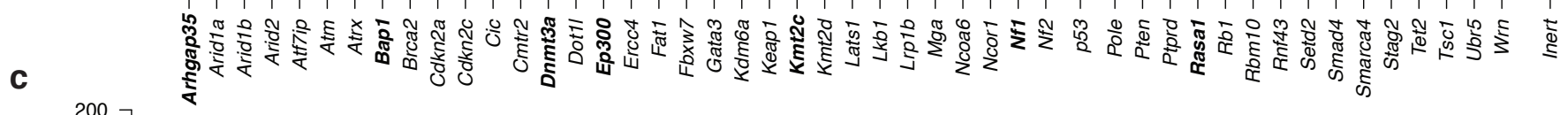

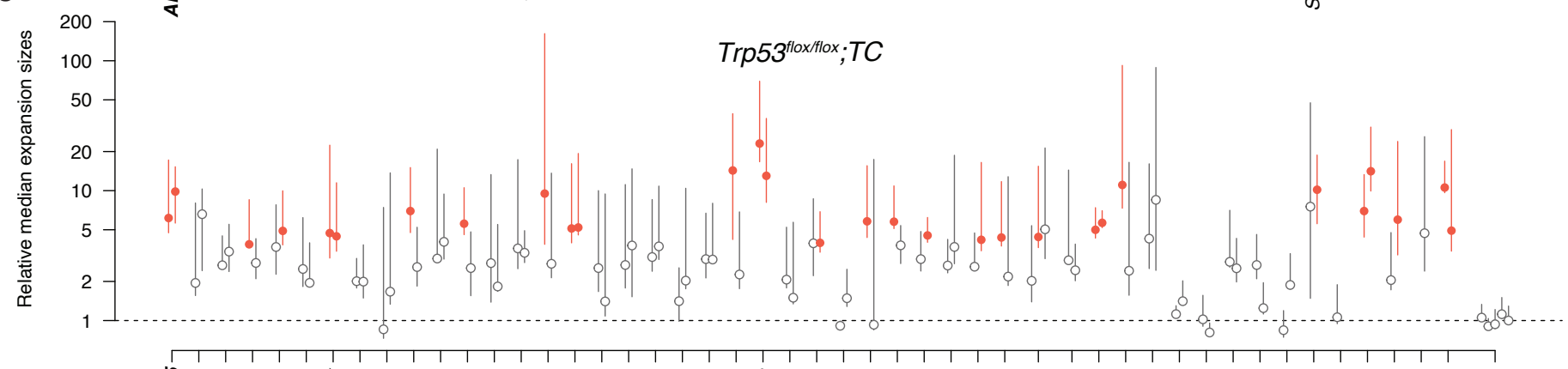

d

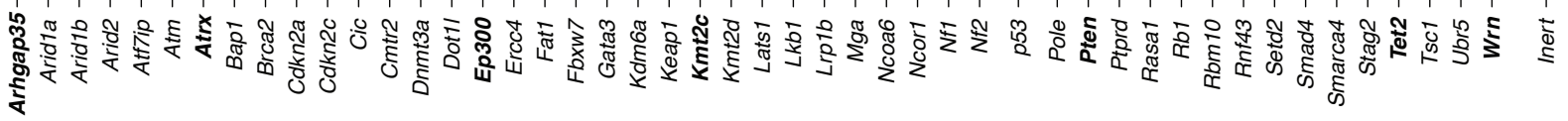

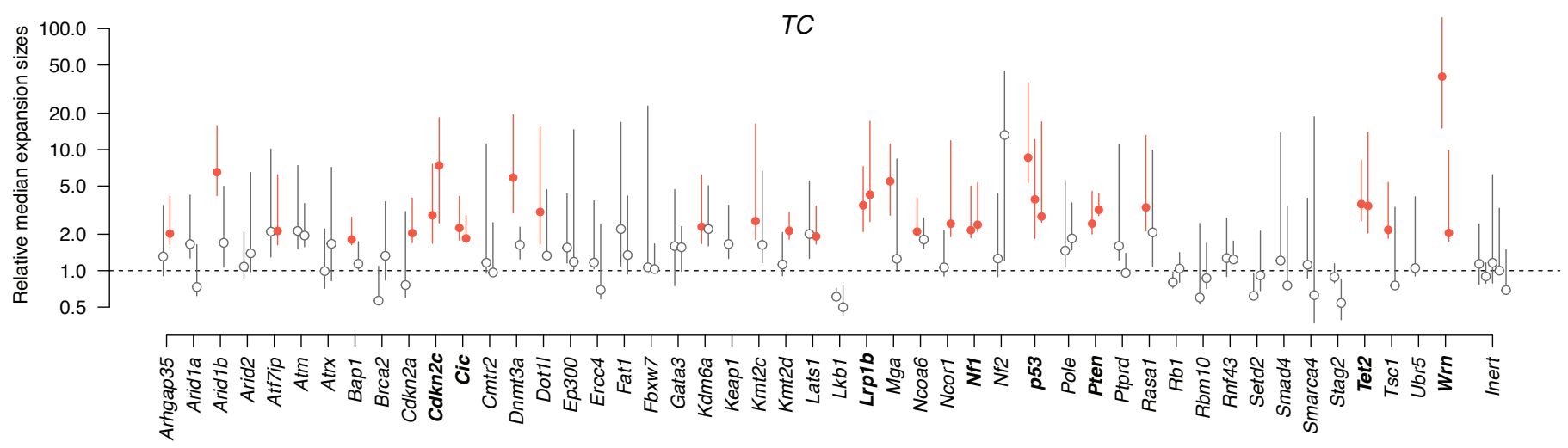

Figure S5. In vivo lung epithelial cell expansion is suppressed by diverse tumor suppressor genes.

a-d. Median cell-expansion sizes (normalized to the sizes of inert sgRNA containing cell expansions) for each putative tumor suppressor targeting sgRNAs in one lung lobe harvested from the indicated mouse genotype are shown. Dotted lines indicate the median value for inert sgRNAs. Cell expansions are defined as clonal expansions containing a minimum of 50 cells. Bootstrap $95 \%$ confidence intervals are shown as whiskers. sgRNAs with median sizes significantly $(p<0.05)$ higher than the median effect for all sgRNAs are shown in red. Gene with significant effect upon inactivation using both sgRNAs are in bold. 
bioRxiv preprint doi: https://doi.org/10.1101/2021.10.20.464849; this version posted December 14, 2021. The copyright holder for this preprint (which was not certified by peer review) is the author/funder. All rights reserved. No reuse allowed without permission. Yousefi, Boross et al.

a

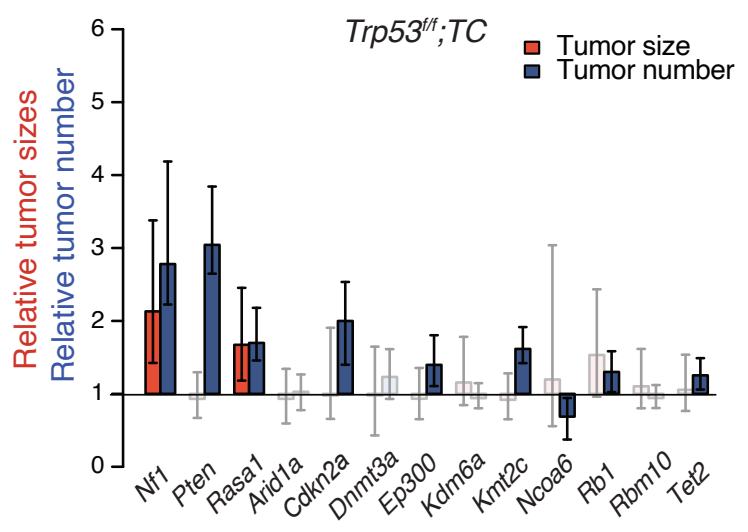

C

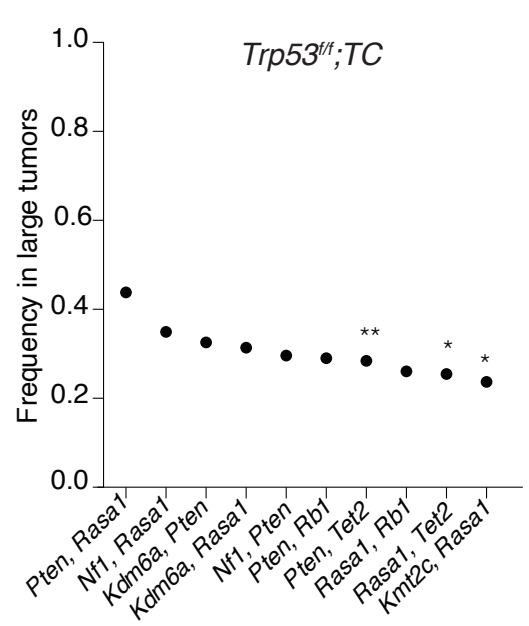

f

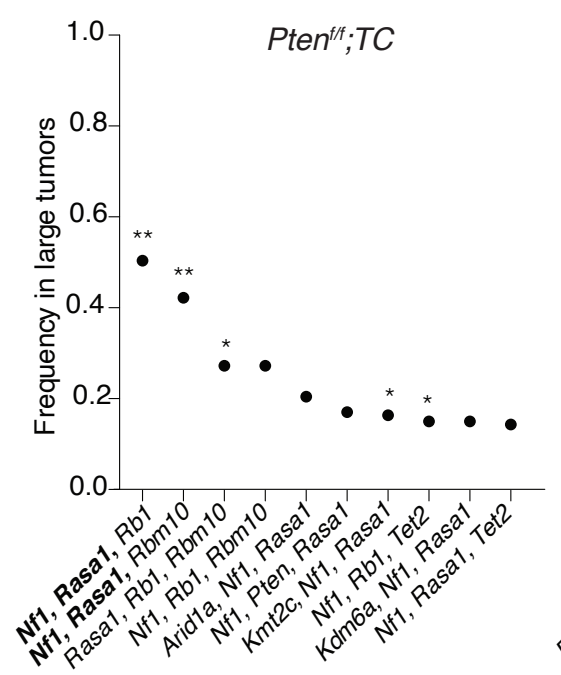

g b

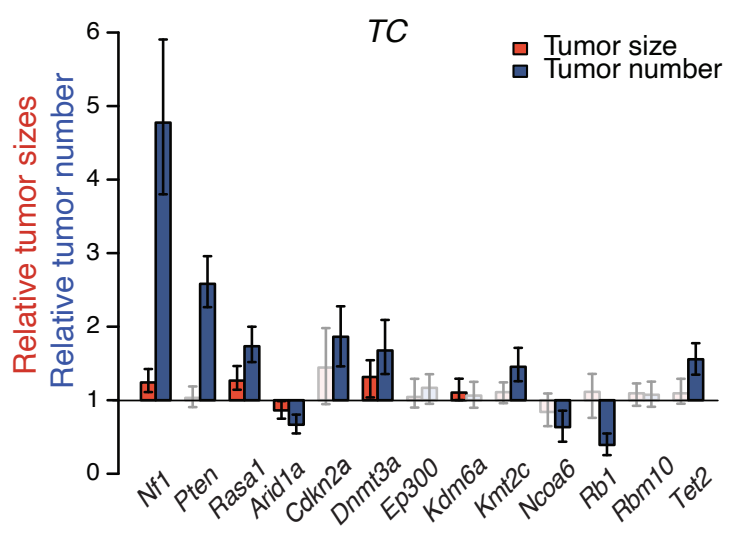

e

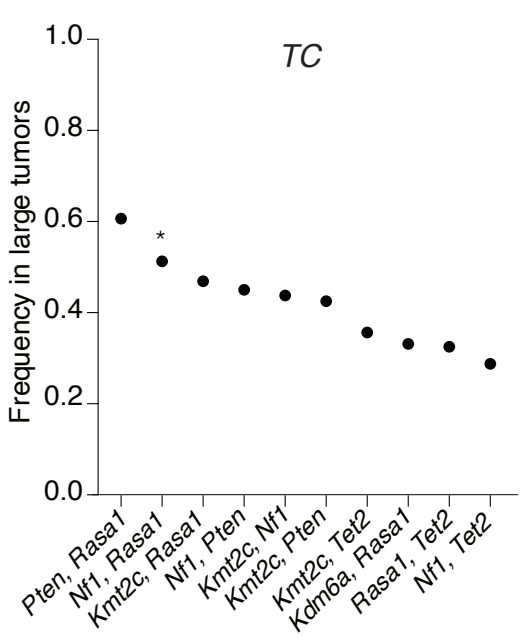

$\mathbf{h}$
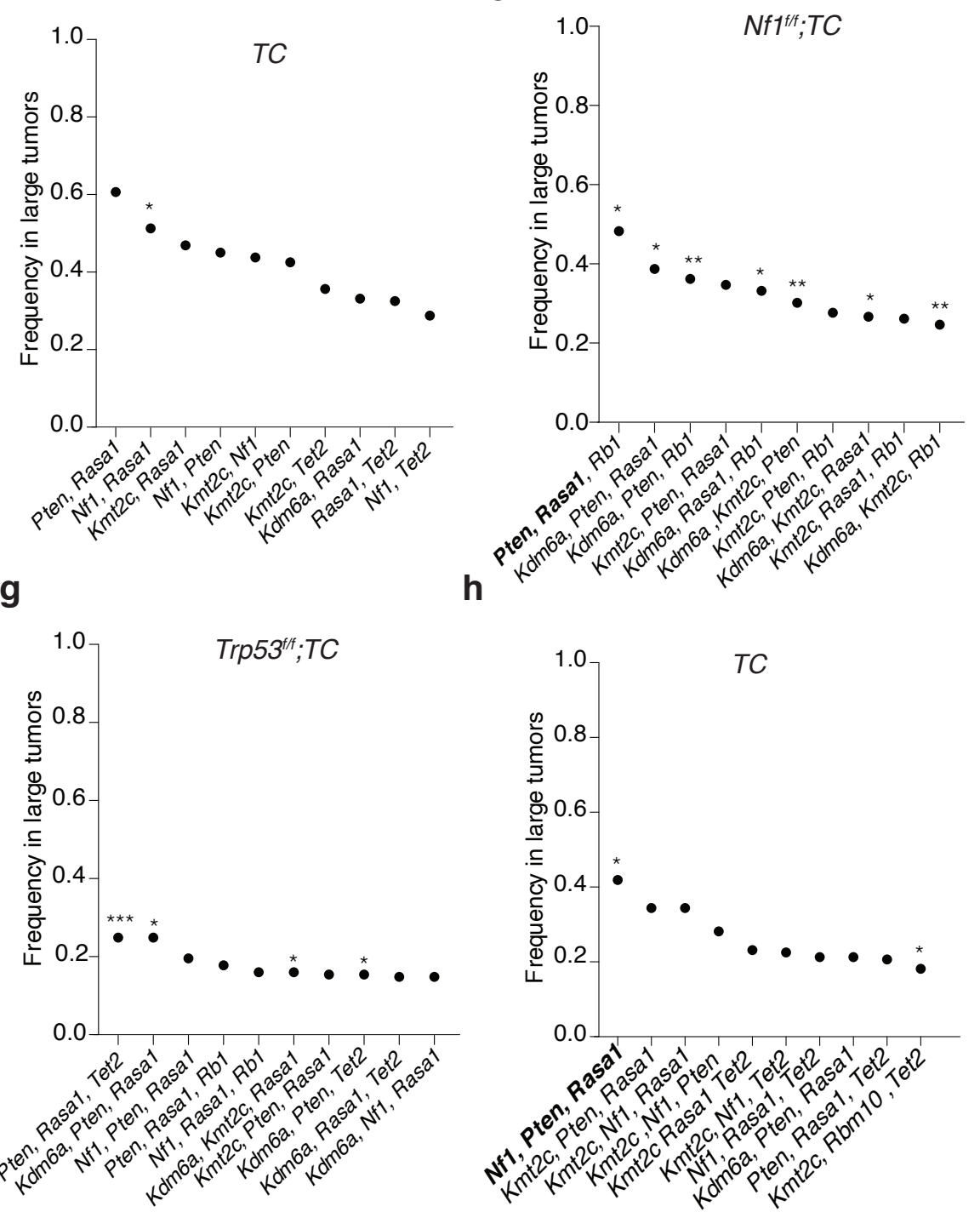

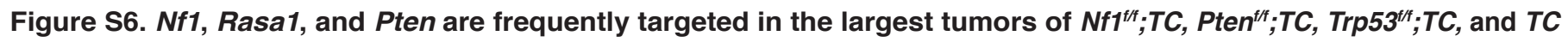
mice.

a, b. The number of tumors with a minimum size of 1000 cells relative to the inert guide is shown as a blue bar. $90^{\text {th }}$ percentile of tumor sizes relative to the inert sgRNA is shown as a red bar. sgRNAs resulting in a significantly higher number or larger tumors than the inert sgRNA $(p<0.05)$ are shown in color. Whiskers show 95\% confidence intervals. Mouse genotypes are indicated on each plot.

c-h. Depiction of the top 10 most frequently co-occurring tumor suppressor alterations in each indicated genotype. Barcodes with the highest cell count in each mouse were investigated for coinfection for multiple viruses (see Methods). The top 10 pairs of tumor suppressors found co-mutated in the largest tumors are shown. ${ }^{*} p<0.05,{ }^{* \star} p<0.01,{ }^{* * *} p<0.001$ based on a permutation test. Combinations of sgRNAs that lead to the generation of Nf1, Rasa1, and Pten mutant cancer cells in a statistically significant manner are in bold. 
bioRxiv preprint doi: https://doi.org/10.1101/2021.10.20.464849; this version posted December 14, 2021. The copyright holder for this preprint (which was not certified by peer review) is the author/funder. All rights reserved. No reuse allowed without permission.

\section{Yousefi, Boross et al.}

a

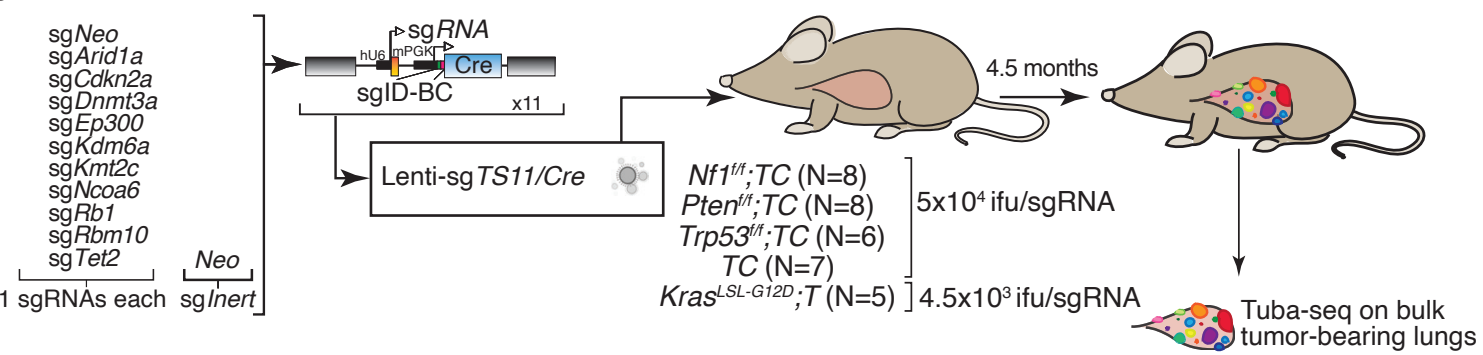

b

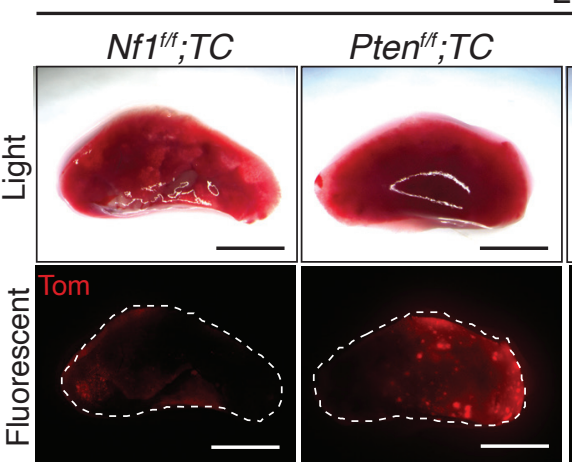

Lenti-sgTS11/Cre

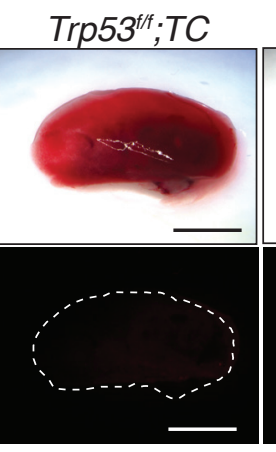

C

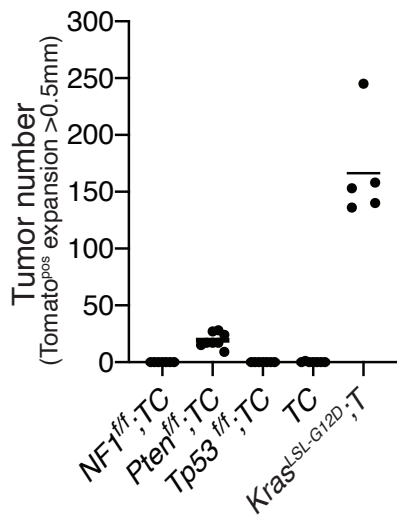

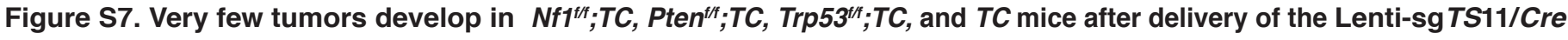
pool.

a. Schematic of tumor initiation with a pool of 11 barcoded Lenti-sgRNA/Cre vectors (Lenti-sgTS11/Cre) similar to Lenti-sgTS14/Cre but excluding the Lenti-sgRNA/Cre vectors with sgNf1, sgRasa1 and sgPten. Each gene is targeted with a single sgRNA. Mouse genotype, mouse number, and titer of virus delivered to each group are indicated. Tuba-seq was performed on each tumor-bearing lung 4.5 months after tumor initiation.

b. Representative light and fluorescence images of lung lobes from the indicated genotypes of mice. Lung lobes are outlined with white dotted lines. Scale bars $=4 \mathrm{~mm}$

c. The number of surface tumors (defined as Tomato-positive expansions larger than $0.5 \mathrm{~mm}$ in diameter) quantified by direct counting. Each dot represents a mouse, and the bar is the mean. 
bioRxiv preprint doi: https://doi.org/10.1101/2021.10.20.464849; this version posted December 14, 2021. The copyright holder for this preprint (which was not certified by peer review) is the author/funder. All rights reserved. No reuse allowed without permission.

Yousefi, Boross et al.

a

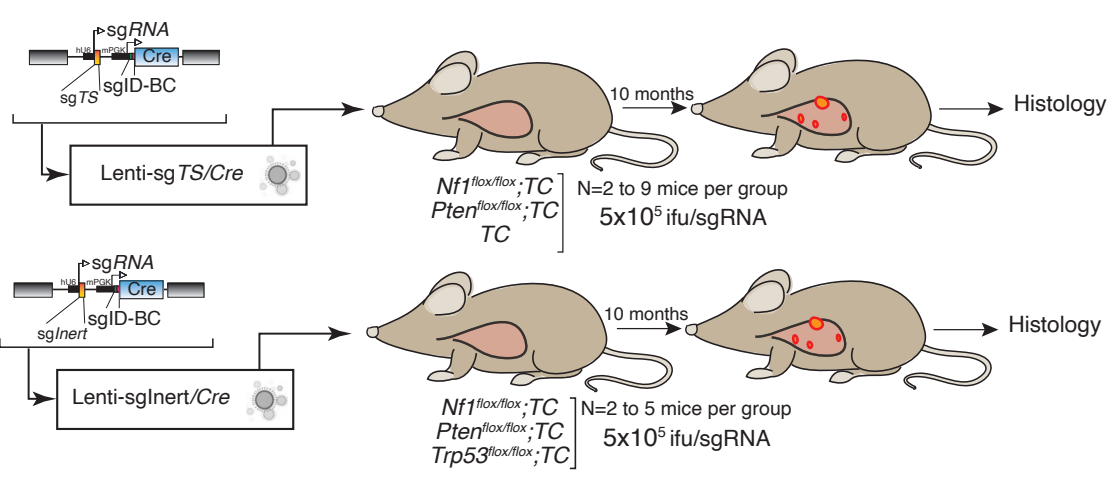

d

C

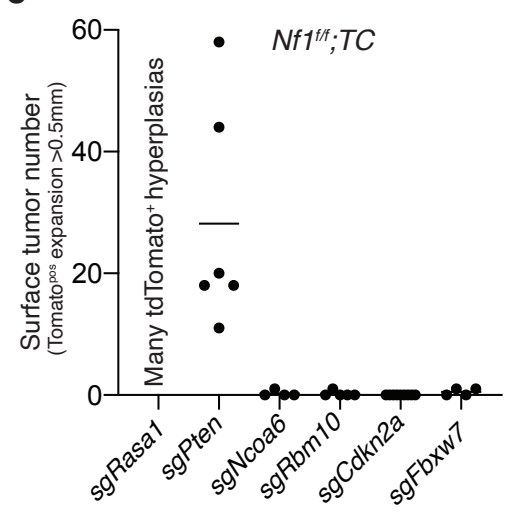

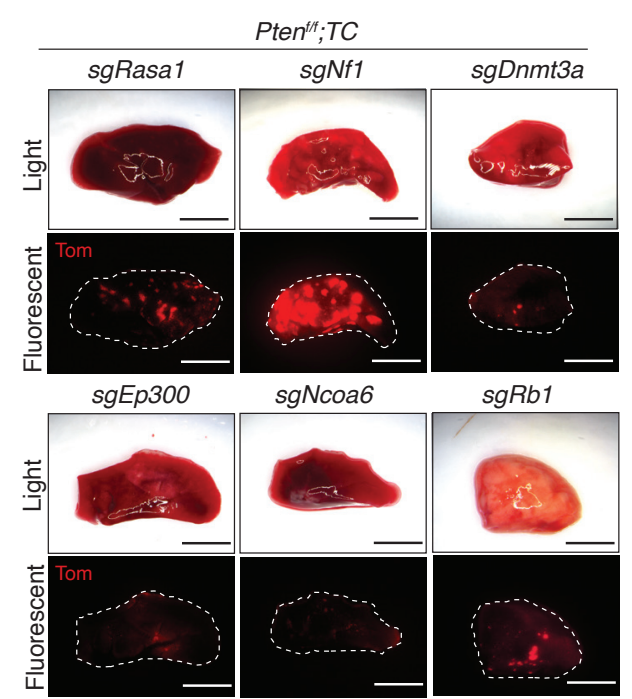

$\mathbf{f}$
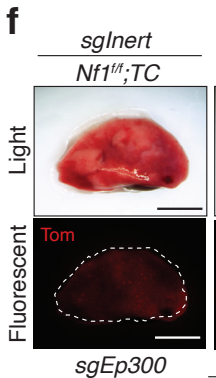

$\operatorname{sgEp300}$

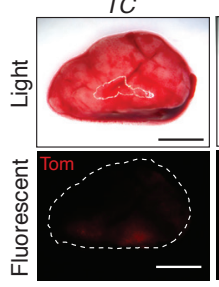

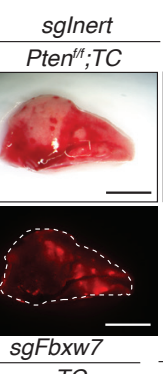

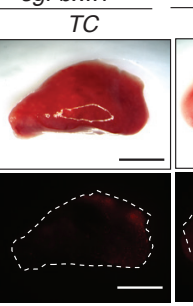

sglnert
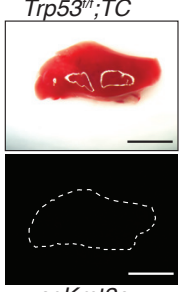

TC

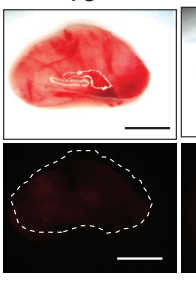

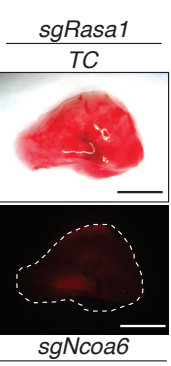
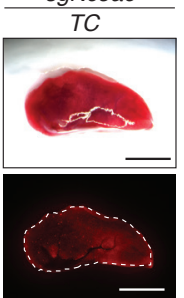

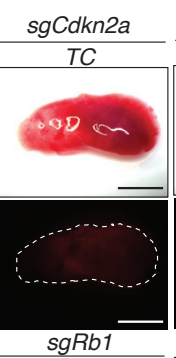

TC

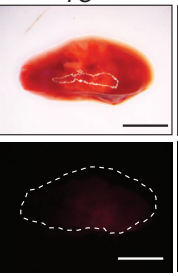

b
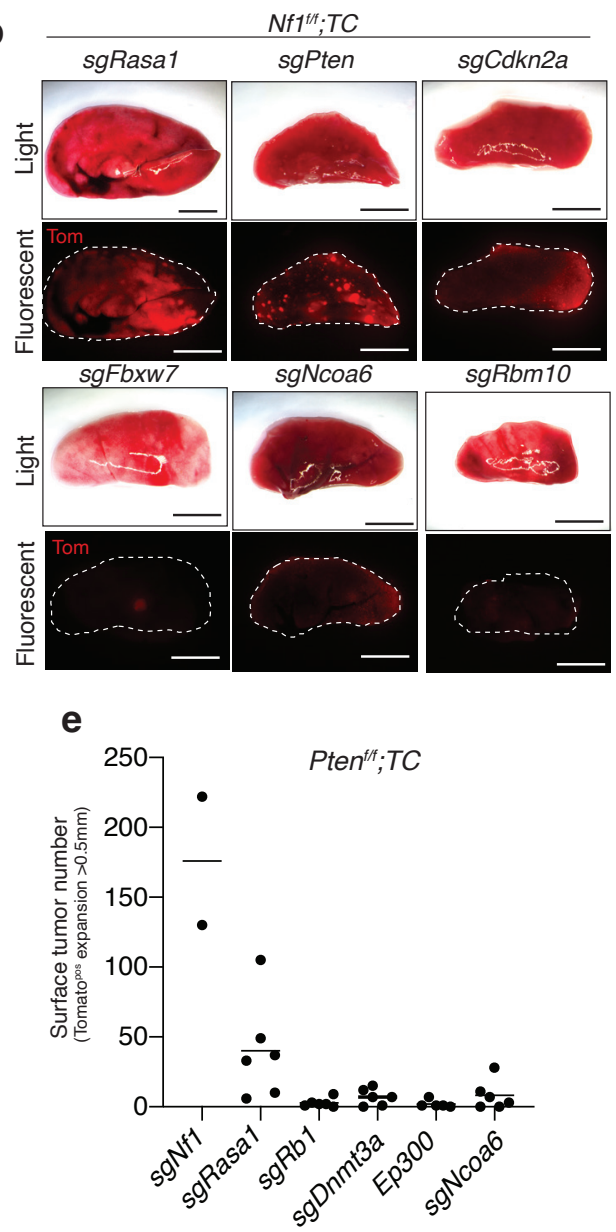

g

Figure S8. Single and Pairwise tumor suppressor gene inactivation is rarely sufficient to generate lung tumors.

a. Schematic of experiments to assess the potential of single or paired tumor suppressor inactivation to generate lung tumors. b,d,f. Representative light and fluorescence images of lung lobes from the indicated genotypes of mice. Lung lobes are outlined with white dotted lines. Scale bar $=4 \mathrm{~mm}$

$\mathbf{c , e , g}$. The numbers of tumors (defined as Tomato-positive cell expansions greater than $0.5 \mathrm{~mm}$ in diameter) was quantified by direct counting. Each dot represents a mouse, and the bar is the mean. The genotypes of the recipient mice and the gene targeted by sgRNA are indicated. Inactivation of Rasa1 in Nf1/ff $T C$ mice in S8b,c generated numerous tdTomato hyperplasias without distinguishable boundaries under the microscope. As a result, surface tumor number was not quantifiable for this group. 
bioRxiv preprint doi: https://doi.org/10.1101/2021.10.20.464849; this version posted December 14,2021 . The copyright holder for this preprint (which was not certified by peer review) is the author/funder. All rights reserved. No reuse allowed without permission. Yousefi, Boross et al.

Lenti-sgRasa1/Cre

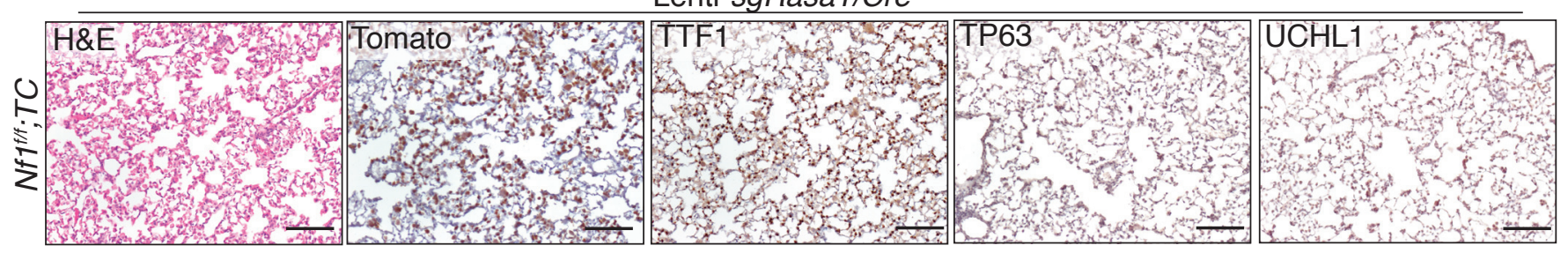

\section{Lenti-sgNf1/Cre}

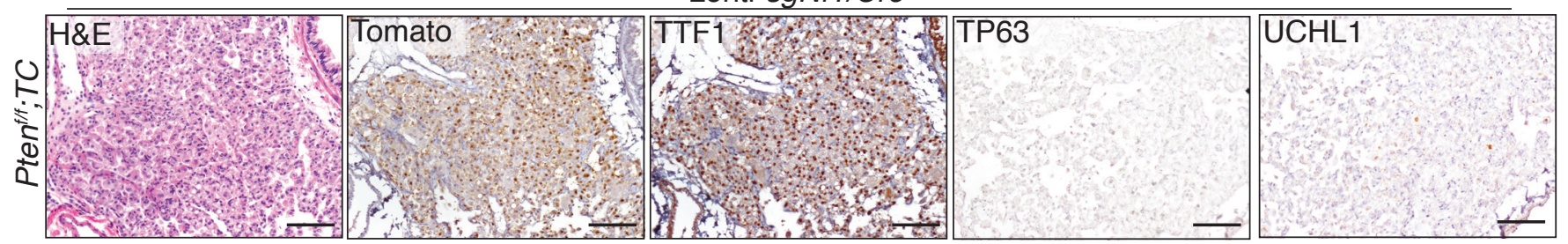

Figure S9. Pairwise inactivation of tumor suppressor genes rarely generates lung adenocarcinomas.

Representative H\&E, Tomato, TTF1, TP63, and UCHL1-stained sections of tumors from Nf1ff; $; C$ and Ptenff;TC mice 10 months after transduction with Lenti-sgRasa1/Cre or Lenti-sgNf1/Cre. Scale bars $=100 \mu \mathrm{m}$ 
bioRxiv preprint doi: https://doi.org/10.1101/2021.10.20.464849; this version posted December 14, 2021. The copyright holder for this preprint (which was not certified by peer review) is the author/funder. All rights reserved. No reuse allowed without permission.

Yousefi, Boross et al.
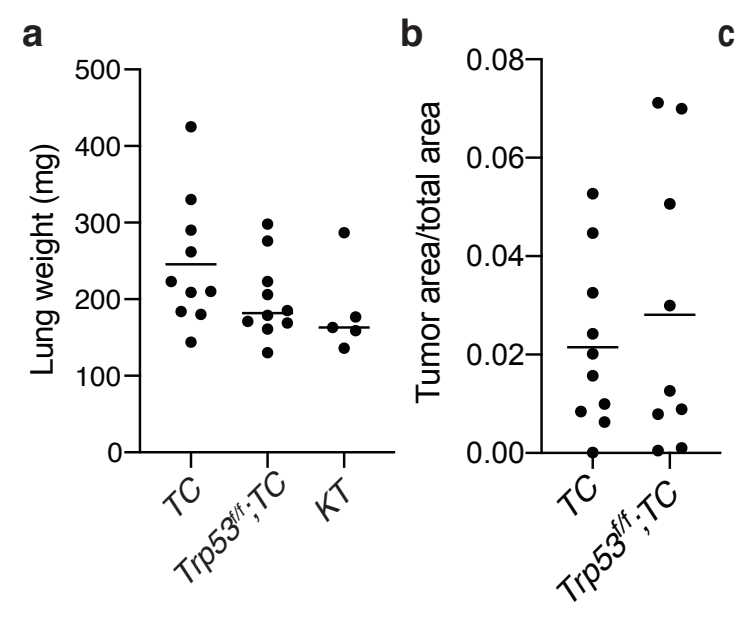

C

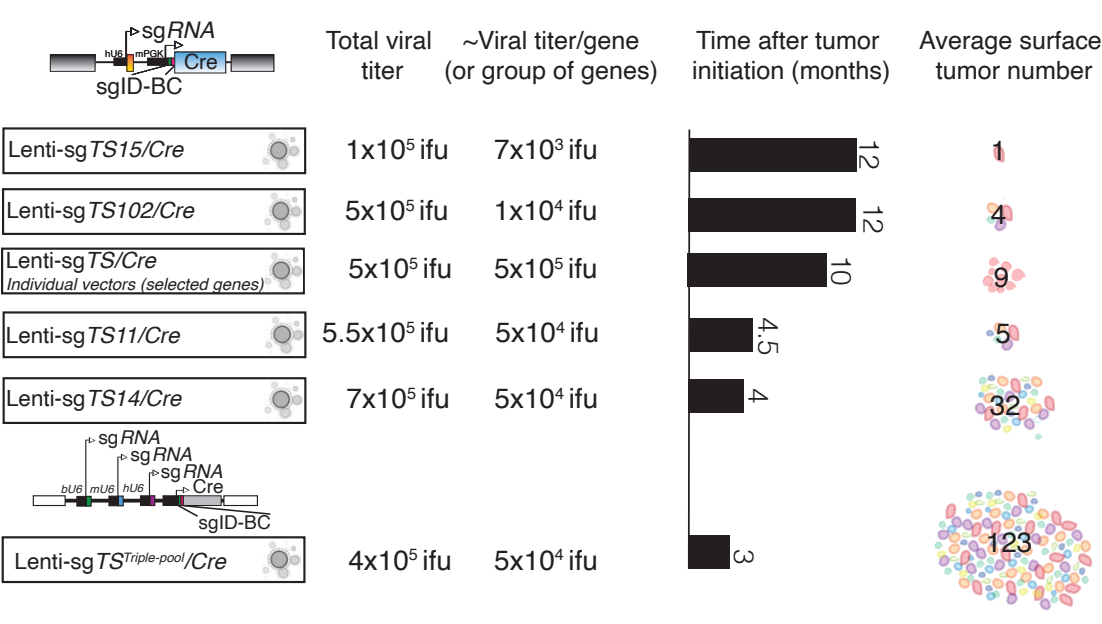

d

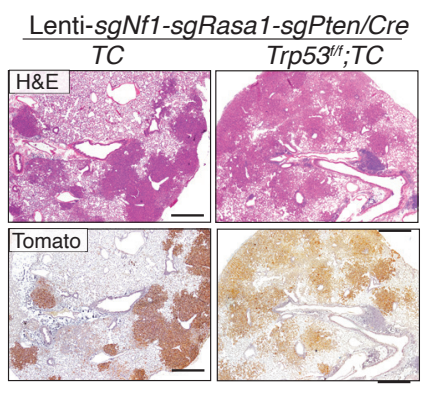

e

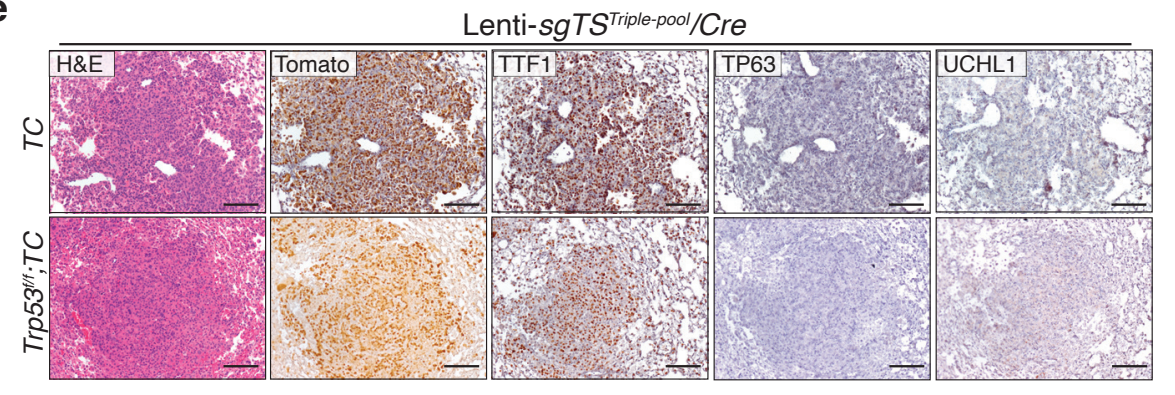

f

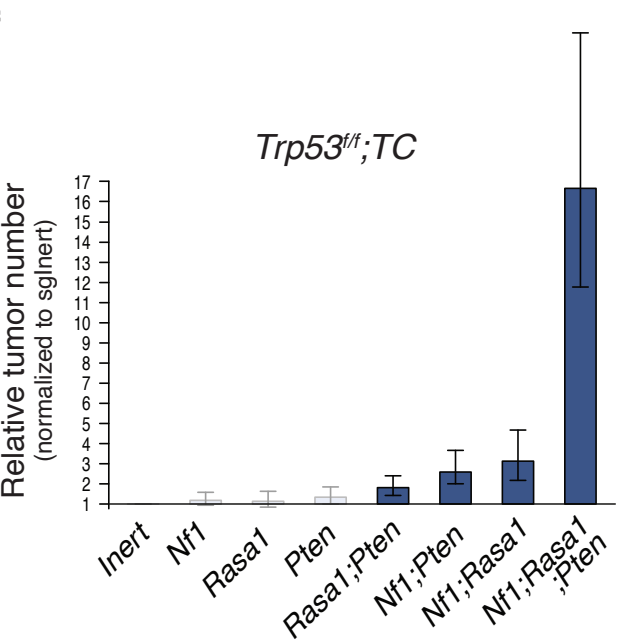

g

$\operatorname{Trp53^{f/f};TC}$

h
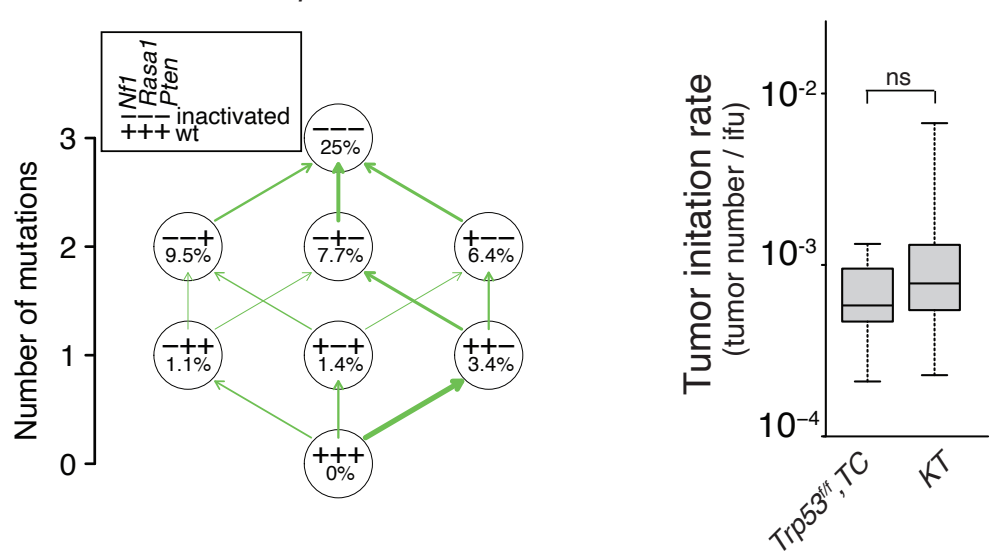

Figure S10. The relative contribution of $\mathbf{N f 1 , ~ R a s a 1 , ~ a n d ~ P t e n ~ i n a c t i v a t i o n ~ t o ~ o n c o g e n e - n e g a t i v e ~ l u n g ~ t u m o r ~ d e v e l o p m e n t ~ i s ~}$ not impacted by Trp53 inactivation.

a. Tumor burden, represented by lung weight. Each dot represents a mouse and the bar is the mean.

b. Quantification of tumor burden based on H\&E images. Each dot represents one lung lobe from each mouse, and the bar is the mean.

c. Schematic of the increase in the number of oncogene-negative lung tumors generated in mice by enriching sgRNAs targeting the most potent tumor suppressor genes in each round of functional genomic screening in vivo. The viral titer, number of months after tumor initiation, and average number of tumors are indicated.

d. Representative H\&E and Tomato stained sections from TC and Trp53 ${ }^{\text {ftoxfiox; }}$ TC mice 3 months after transduction with Triple-Lenti-sgNf1-sgRasa1-sgPten/Cre. Scale bar $=500 \mu \mathrm{m}$

e.Representative H\&E, Tomato, TTF1, TP63, and UCHL1-stained sections of tumors from TC and Trp53 $3^{\text {ftoxffox}}$; TC mice 3 months after transduction with Lenti-sgTS ${ }^{\text {Triple-pool} / C r e . ~ S c a l e ~ b a r ~}=100 \mu \mathrm{m}$

f. Numbers of tumors (with $>1000$ neoplastic cells) are shown relative to Inert sgRNA. sgRNAs resulting in a significantly higher number of tumors than sglnert $(p<0.05)$ are shown in color. Mean $+/-95 \%$ confidence interval is shown.

g. Adaptive landscape of Nf1, Rasa1, and Pten inactivation in Trp53 fioxffox; TC is shown. Nodes represent genotypes shown as a string of +(wild-type) and - (inactivated) symbols representing Nf1, Rasa1, and Pten. Numbers in the nodes indicate fitness increase compared to wild-type. The relative probability of each beneficial mutation is shown as arrow widths (see Methods).

h. Quantification of the ability of combined Nf1/Rasa1/Pten inactivation in TC mice and oncogenic Kras ${ }^{\mathrm{G} 12 \mathrm{D}}$ in $K T$ mice to initiate tumors. Number of tumors (with $>1000$ neoplastic cells) per infectious unit (ifu) is shown. The bar is the median, the box represents the interquartile range, and the whiskers show minimum and maximum values. n.s: non-significant 
bioRxiv preprint doi: https://doi.org/10.1101/2021.10.20.464849; this version posted December 14, 2021. The copyright holder for this preprint (which was not certified by peer review) is the author/funder. All rights reserved. No reuse allowed without permission. Yousefi, Boross et al.

a

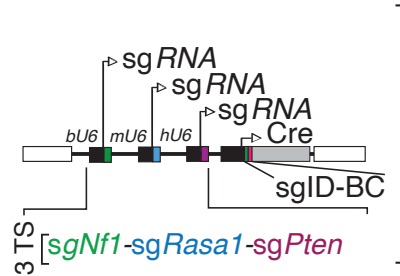

b

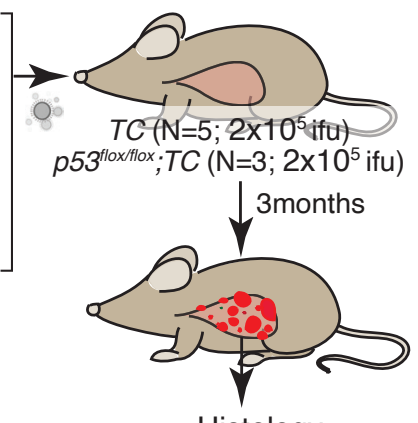

Histology

Whole-exome sequencing

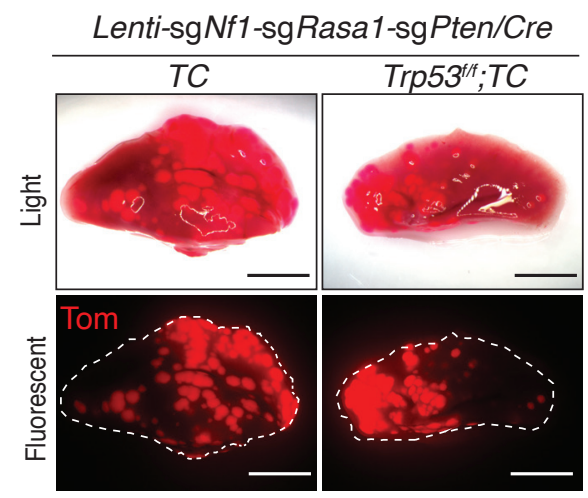

C

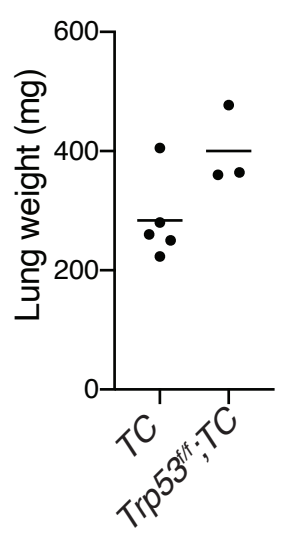

d

e

Lenti-sgNf1-sgRasa1-sgPten/Cre

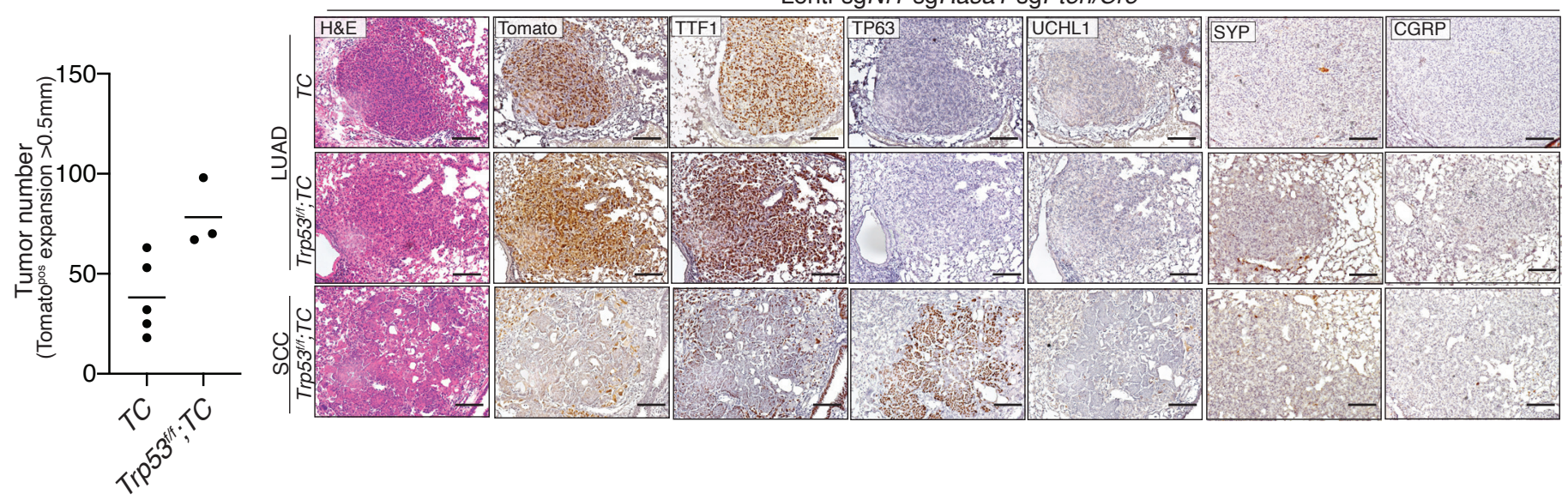

f

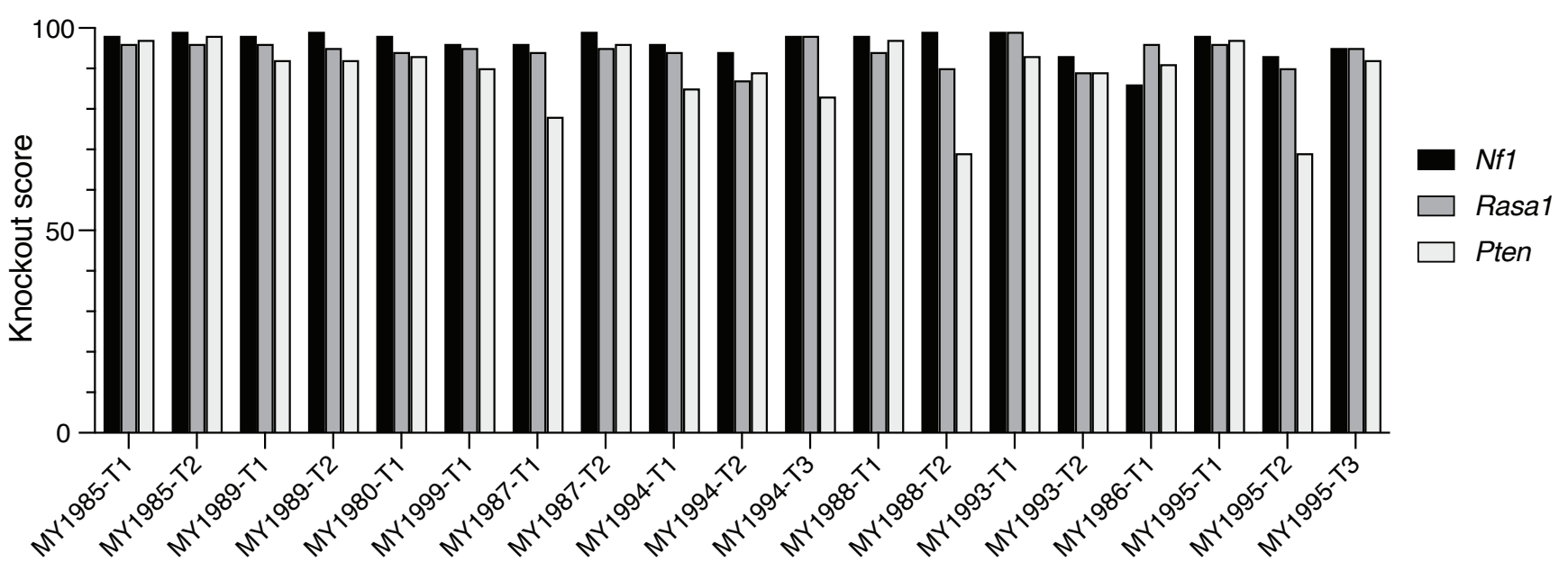

Figure S11. Oncogene-negative lung tumors driven by inactivation of $\mathbf{N f 1 , ~ R a s a 1 , ~ a n d ~ P t e n ~ a r e ~ a l m o s t ~ e x c l u s i v e l y ~ a d e n o - ~}$ mas/adenocarcinoma.

a. Schematic of inactivation of Nf1, Rasa1, and Pten in TC and Trp53 fioxfiox; TC mice utilizing triple guide vectors and CRISPR/Cas9mediated gene-inactivation. Mouse genotype, mouse number, and titer of virus delivered to each mouse are indicated. ifu, infection unit b. Bright-field and fluorescence images of lungs from the indicated mice 3 months after tumor initiation with Lenti-sgNf1-sgRasa1-sgPten/Cre virus. Lung lobes are outlined with a dashed white line. Scale bars $=4 \mathrm{~mm}$

c. Tumor burden, represented by lung weight. Each dot represents a mouse, and the bar is the mean.

d. Quantification of tumor number based on H\&E images of one lung lobe from each mouse. Each dot represents one lung lobe from each mouse, and the bar is the mean.

e. Representative H\&E, Tomato, TTF1, TP63, UCHL1, SYNAPTOPHYSIN (SYP), and CGRP-stained tumor sections from TC and Trp53floxflox; TC mice 3 months after transduction with Lenti-sgNf1-sgRasa1-sgPten/Cre. Squamous cell lung cancer was only rarely observed in Trp53 floxflox; $T$ TC mice (3 out 264 tumors). Scale bars $=100 \mu \mathrm{m}$

f. Analysis of insertion and deletion in genomic DNA from FACS sorted tumors of 19 TC mice 4 months after transduction with $5 \times 10^{4}$ ifu of Lenti-sgNf1-sgRasa1-sgPten/Cre. sgRNA targeted regions were PCR amplified, and knockout scores, representing the proportion of cells that have either a frameshift-inducing indel or a large indel in a protein-coding region, were calculated using Synthego's ICE. 
bioRxiv preprint doi: https://doi.org/10.1101/2021.10.20.464849; this version posted December 14, 2021. The copyright holder for this preprint (which was not certified by peer review) is the author/funder. All rights reserved. No reuse allowed without permission.

a

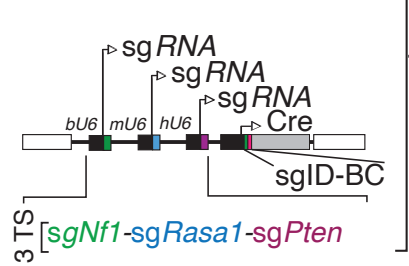

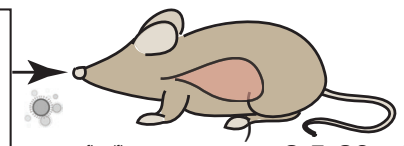

$\operatorname{Trp5} 3^{\text {floxfliox}} ; T C\left(\mathrm{~N}=32 ; 2.5-20 \times 10^{3}\right.$ ifu $)$

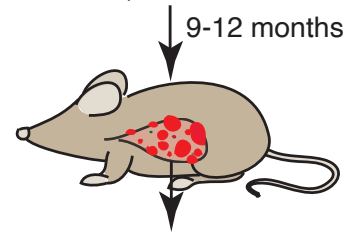

Histology

Whole-exome sequencing b

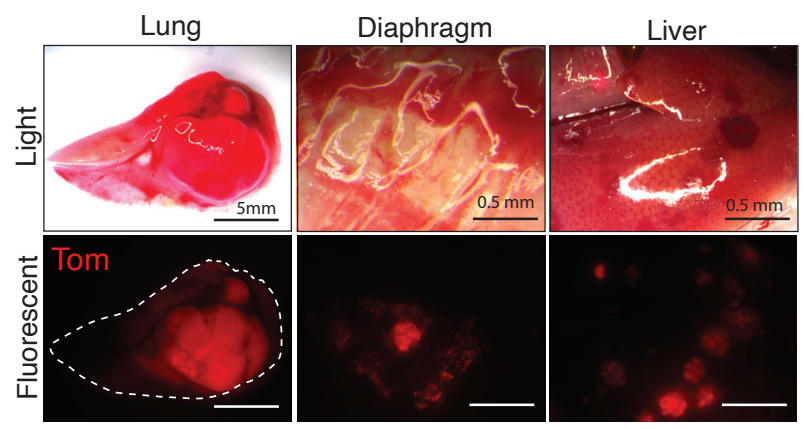

C
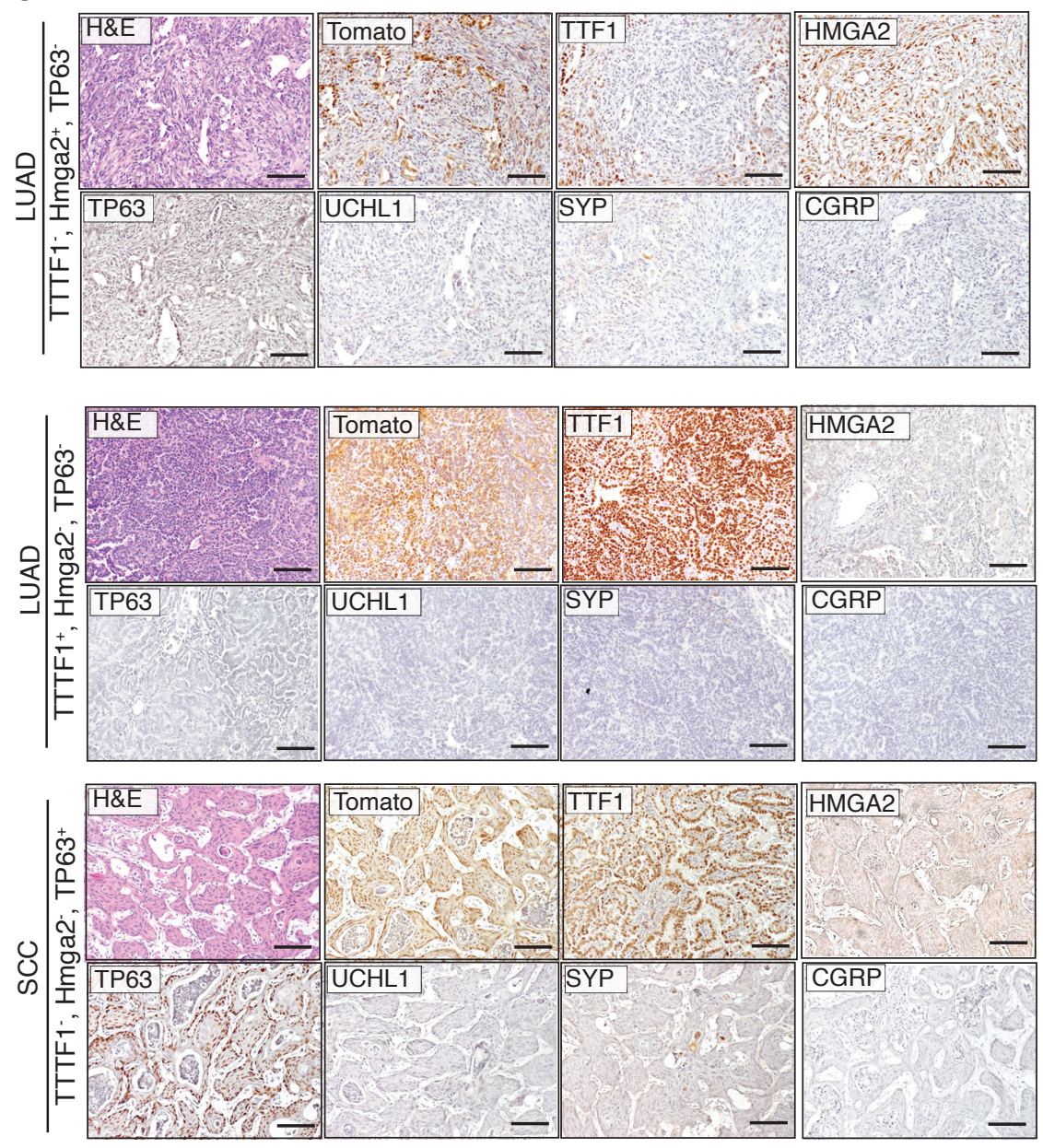

d

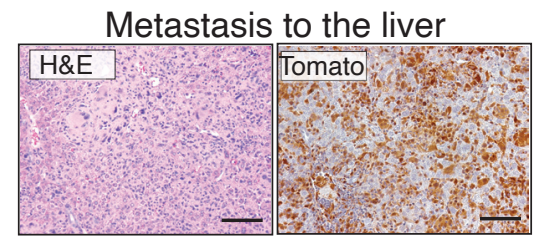

Figure S12. Inactivation of Nf1, Rasa1, and Pten generates lung tumors with the ability to metastasize to other organs. a. Schematic of inactivation of Nf1, Rasa1, and Pten in Trp53 floxflox;TC mice using the Lenti-sgNf1-sgRasa1-sgPten/Cre vector. Mouse genotype, mouse number, and titer of virus delivered mice are indicated. ifu, infection unit

b. Bright-field and fluorescence images of lungs, diphragm, and liver from the Trp53 ${ }^{\text {floxfllox}} ; T$ TC mice 12 months after tumor initiation with Lenti-sgNf1-sgRasa1-sgPten/Cre virus. Lung lobes are outlined with a dashed white line. Scale bars=5 and $0.5 \mathrm{~mm}$ (4 out of $32 \mathrm{mice}$ had obvious metastasis).

c. Representative H\&E, Tomato, TTF1, HMGA2, TP63, UCHL1, SYNAPTOPHYSIN (SYP), and CGRP-stained tumor sections from Trp53 floxflox; TC mice 9-12 months after transduction with Lenti-sgNf1-sgRasa1-sgPten/Cre. Scale bars $=100 \mu \mathrm{m}$.

d. H\&E and tdTomato staining of liver sections from one of the Trp53 floxflox; $T C$ mice with metastasis. Scale bars $=100 \mu \mathrm{m}$. 
bioRxiv preprint doi: https://doi.org/10.1101/2021.10.20.464849; this version posted December 14,2021 . The copyright holder for this preprint (which was not certified by peer review) is the author/funder. All rights reserved. No reuse allowed without permission.

Yousefi, Boross et al.

a

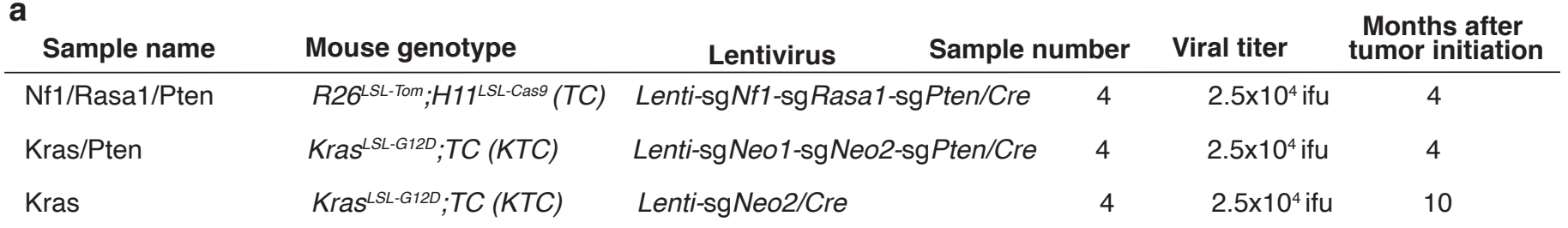

b

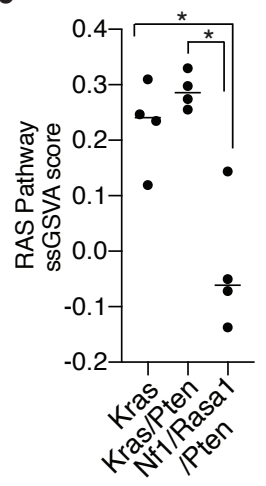

c

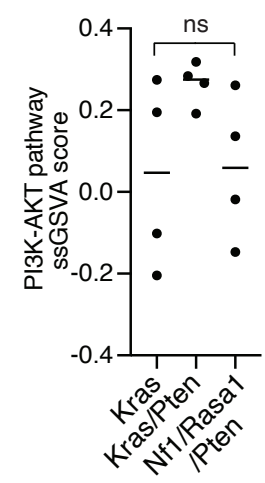

d


f
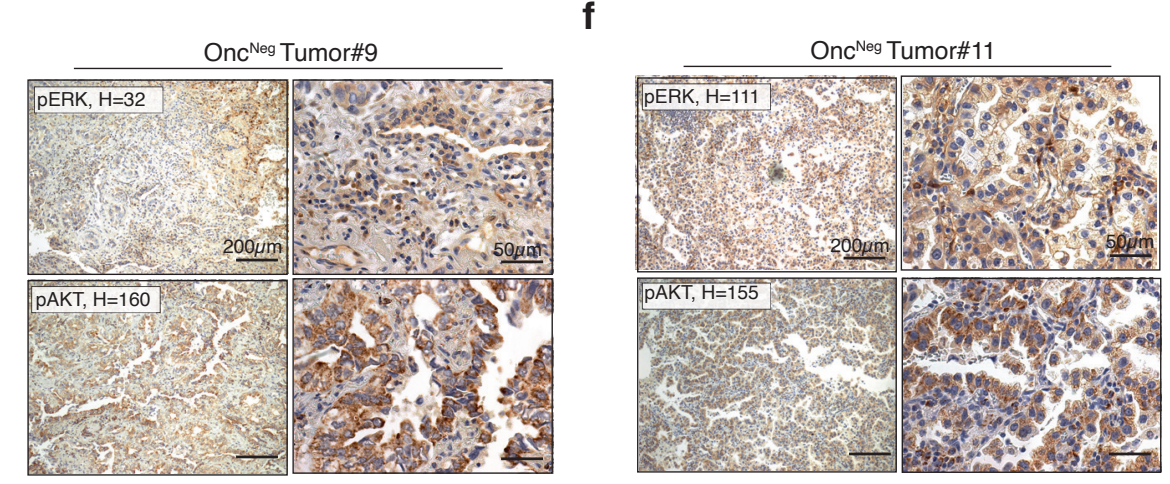

g

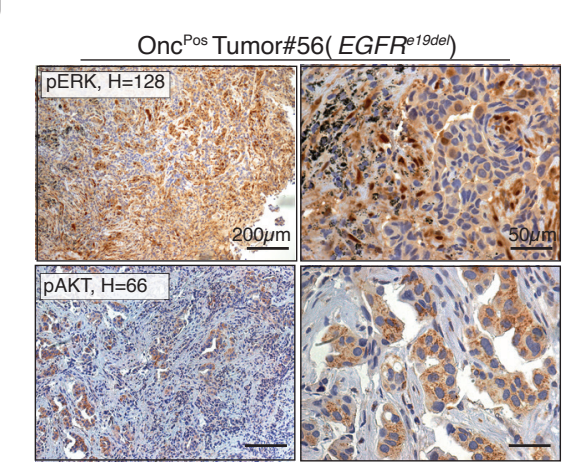

h

\section{i}

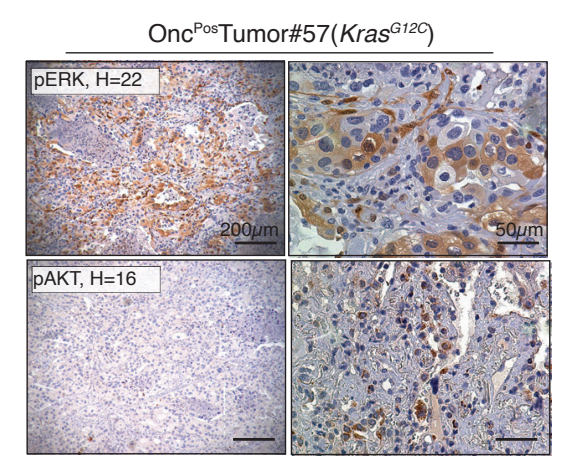

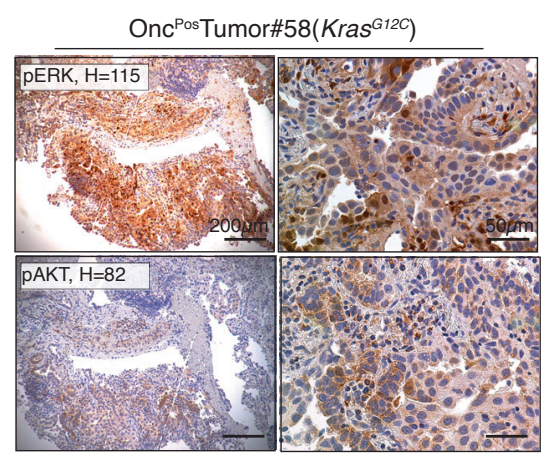

j

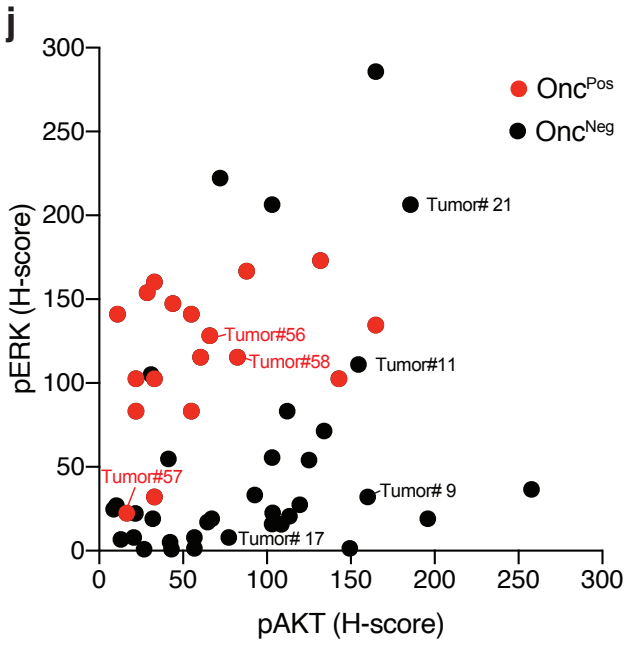

Figure S13. Onc-negative ${ }^{\mathrm{RAS} / \mathrm{PI} 3 \mathrm{~K}}$ subtype of lung adenocarcinomas activate RAS and PI3K pathways biochemically.

a. Summary of the mouse tumors sorted using FACS and analyzed by RNA-sequencing and immunohistochemistry.

b,c. RAS and PI3K-AKT pathway gene-set profiles estimated by single-sample Gene Set Enrichment Analysis (SSGSVA). Tumors from Kras ${ }^{G 12 D}$;TC (KTC+ sgInert and KTC+sgPten: Kras and Kras/Pten) mice are compared with Nf1, Rasa1, and Pten mutant tumors(Nf1/Rasa1/Pten). The bar is the mean. ns: non-significant, " $\mathrm{p}<0.05$ using Mann-Whitney U test.

d. Representative immunohistochemistry for pERK and PAKT to determine activation of RAS and PI3K pathway in tumors with the indicated genotypes. The first gene is mutated using floxed alleles, and the second gene is inactivated using sgRNA/Cas9 (see Figure $\mathbf{S 8}$ for more details). Scale bar $=50 \mu \mathrm{m}$ e-i. Representative pAKT and pERK-stained sections of tumors from human oncogene-negative and oncogene-positive tumors. $\mathrm{H}$-score for the whole section is indicated on each representative image. Scale bar $=200 \mu \mathrm{M}$ (right), $50 \mu \mathrm{m}$ (left)

j. Replotting of pAKT and pERK staining on 35 oncogene-negative and 18 oncogene-positive human lung adenocarcinomas (Figure 4f, g). The tumors shown as IHC examples in Figure $\mathbf{4 d , e , ~ a n d ~} \mathbf{S 1 4 e - i}$ are labeled on this plot. 
bioRxiv preprint doi: https://doi.org/10.1101/2021.10.20.464849; this version posted December 14, 2021. The copyright holder for this preprint (which was not certified by peer review) is the author/funder. All rights reserved. No reuse allowed without permission.

Yousefi, Boross et al.

a

b
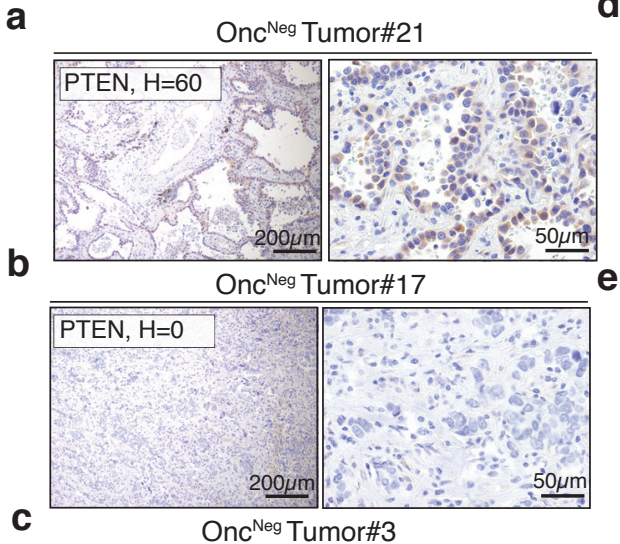

c

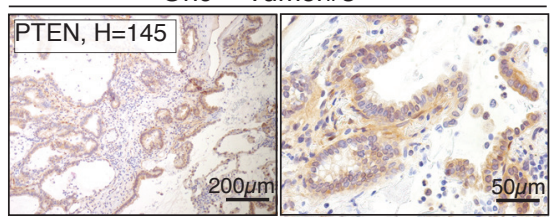

d

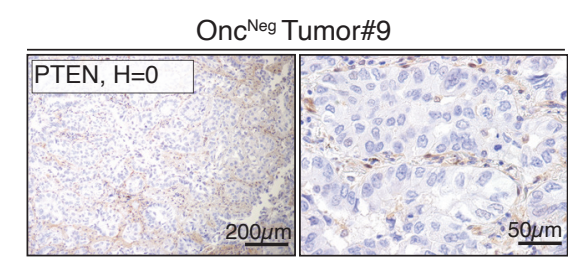

e

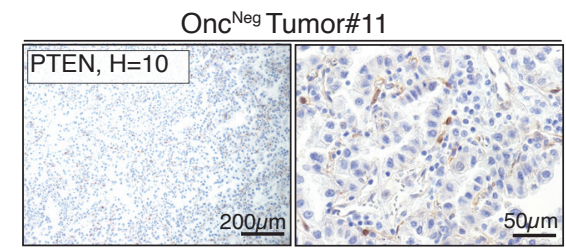

f

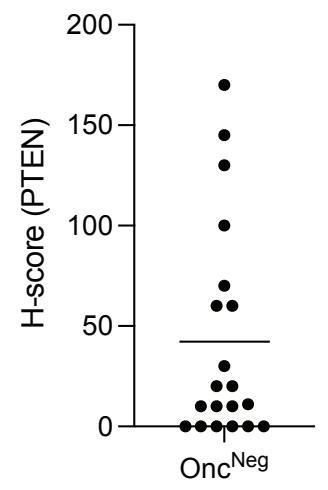

\section{g}

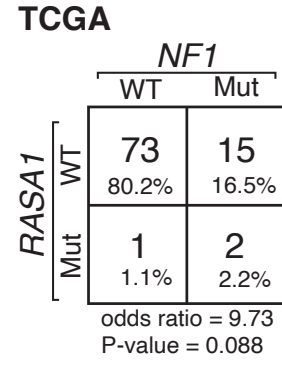

h

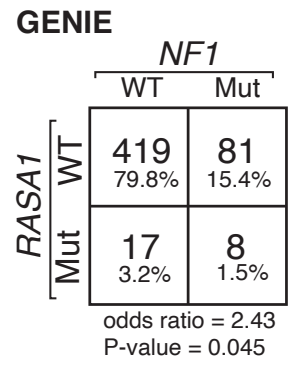

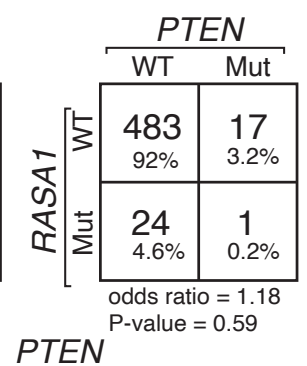

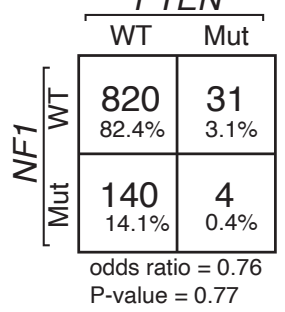

i

GENIE

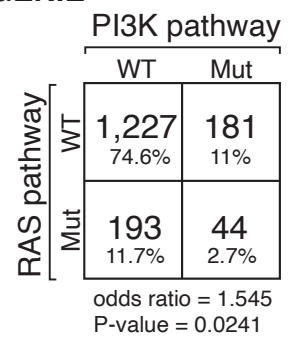

Figure S14. Alterations in RAS and PI3K pathways are enriched in Onc-negative ${ }^{\mathrm{RAS} / \mathrm{P} / 3 \mathrm{~K}}$ subtype of human lung adenocarcinomas.

a-e. Representative PTEN-stained sections of oncogene-negative human tumors. $\mathrm{H}$-score for the whole section is indicated for each representative image. Scale bar $=200 \mu \mathrm{M}$ (right), $50 \mu \mathrm{m}$ (left)

f. PTEN H-scores for oncogene-negative human lung adenocarcinoma tumors.

g, h. Alteration frequencies of NF1, RASA1, and PTEN (point mutation, CNV, and indel) and assessment of their co-occurrences, the p-values were calculated by two-sided Fisher's Exact Test. 91 oncogene-negative tumors were from the TCGA datasets. 525, 995, and 525 tumors were analyzed for RASA1/PTEN, NF1/PTEN, and RASA1/NF1 alterations from the GENIE dataset.

i. Frequency of alteration of well-established components of RAS and PI3K pathways (Table S6) queried in GENIE data set and their co-occurrences, the p-value calculated by two-sided Fisher's Exact Test. 
bioRxiv preprint doi: https://doi.org/10.1101/2021.10.20.464849; this version posted December 14, 2021. The copyright holder for this preprint (which was not certified by peer review) is the author/funder. All rights reserved. No reuse allowed without permission.

Yousefi, Boross et al.

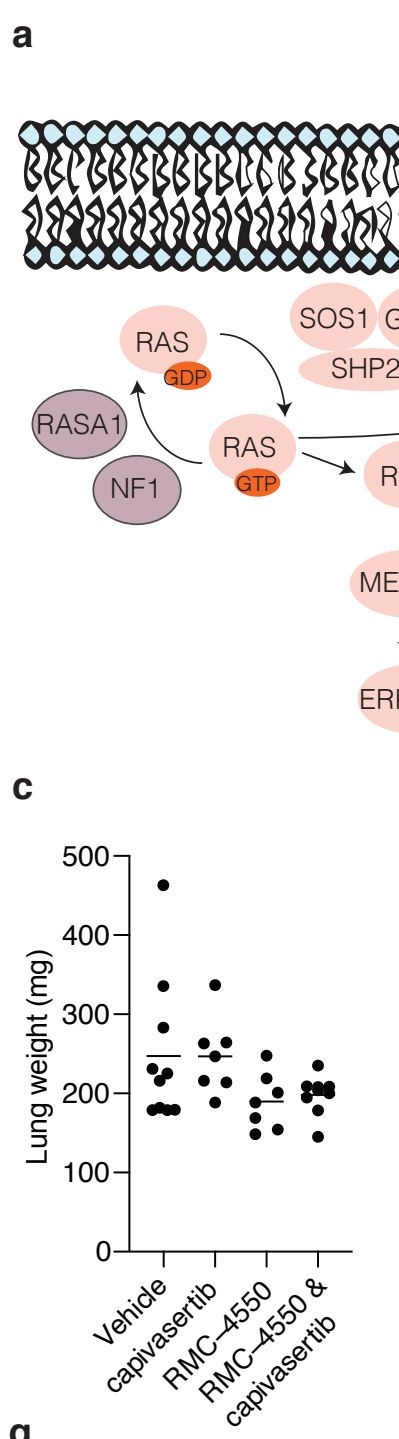

g

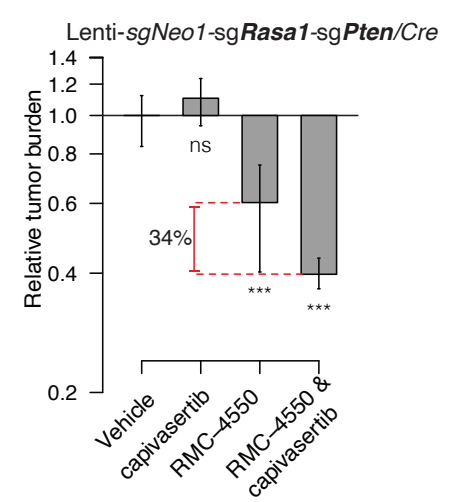

b

Drug Molecular target Dosage

\begin{tabular}{cc}
\hline RMC-4550 SHP2 & Day 1-8: $30 \mathrm{mg} / \mathrm{kg}$ \\
& Day 9-10:No treatment \\
& Day 11-14: $30 \mathrm{mg} / \mathrm{kg}$
\end{tabular}

capivasertib AKT1/2/3 Day 1-8: $100 \mathrm{mg} / \mathrm{kg}$

Day 9-10: No treatment

Day 11-14: $100 \mathrm{mg} / \mathrm{kg}$

RMC-4550 \& SHP2 \& Day 1-8: $30 \mathrm{mg} / \mathrm{kg}$ and $100 \mathrm{mg} / \mathrm{kg}$ capivasertib AKT1/2/3 Day 9-10: No treatment

Day 11-14: $15 \mathrm{mg} / \mathrm{kg}$ and $50 \mathrm{mg} / \mathrm{kg}$

\section{f}
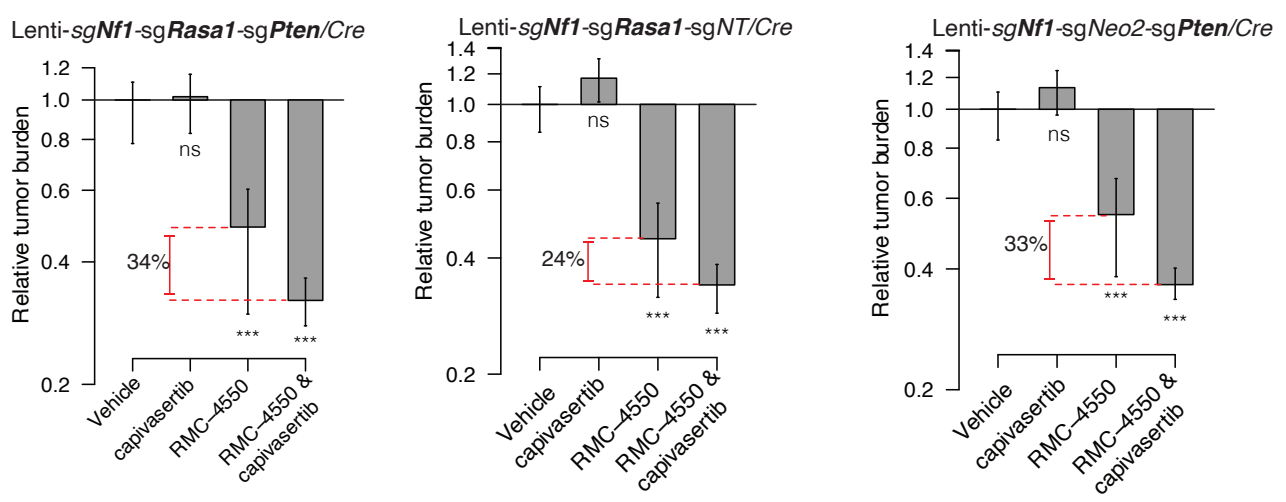

h

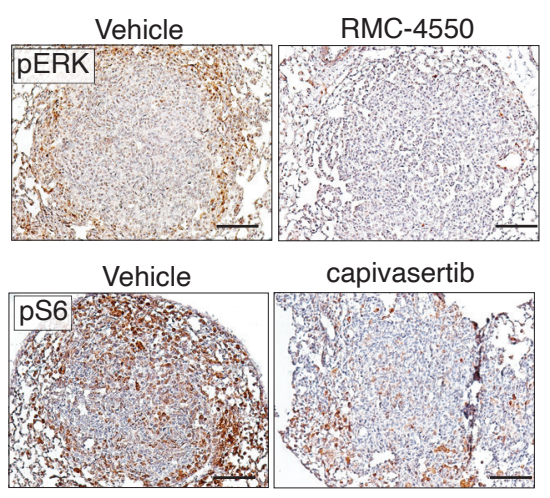

Figure S15. Nf1, Rasa1, and Pten mutant oncogene-negative lung tumors respond to inhibition of PI3K and RAS pathways.

a. RAS and PI3K pathways are activated by alterations of Nf1, Rasa1, and Pten and targeted by SHP2 and AKT inhibitors.

b. Drugs used to inhibit RAS and PI3K pathways in vivo and their dosages.

c. Lung weight of mice described in Figure 5a-b.

d-g. Relative tumor burden in mice after treatment with capivasertib, RMC-4550, and combination of these two drugs compared with tumor burden in vehicle-treated mice. ns: non-significant, ${ }^{* \star *} p<0.001$. Drug response is shown for tumors driven by inactivation of different combinations of Nf1, Rasa1, and Pten.

h. Representative $p E R K$ and pS6-stained sections of oncogene-negative ${ }^{\text {RASIPIKK }}$ tumors from TC mice described in S16 after treatment with the indicated drugs. The mice were injected with one last dose of indicated drugs 4 hours before tissue harvest. Scale bars $=100 \mu \mathrm{m}$ 
bioRxiv preprint doi: https://doi.org/10.1101/2021.10.20.464849; this version posted December 14, 2021. The copyright holder for this preprint (which was not certified by peer review) is the author/funder. All rights reserved. No reuse allowed without permission.

Yousefi, Boross et al.

a

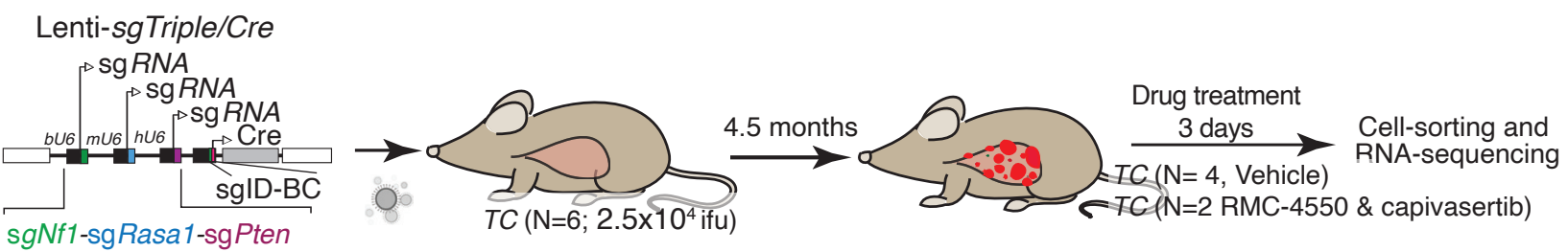

b

C

d

$\mathbf{e}$
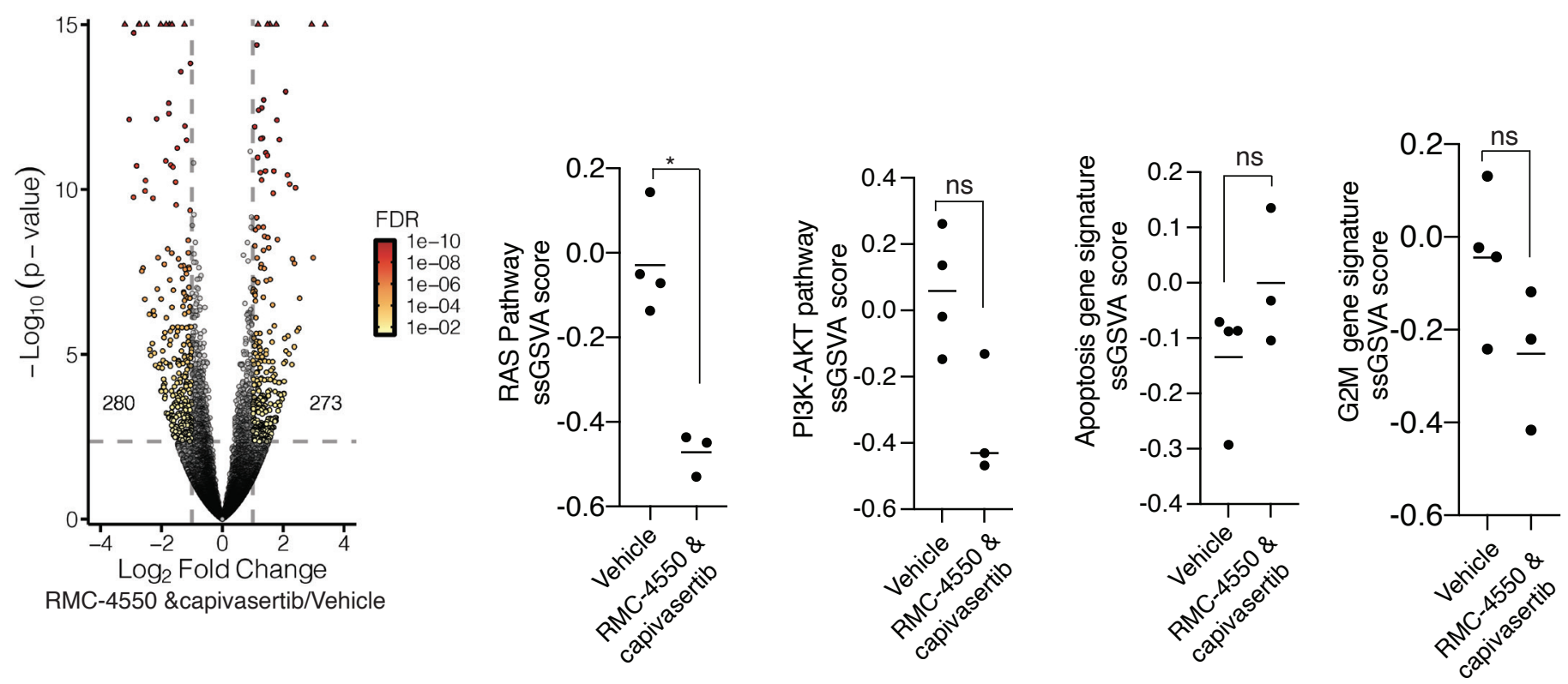

Figure S16. RMC-4550 and capivasertib treatment induces apoptosis gene signature and suppresses G2/M gene signature in Onc-negative $^{\text {RAS/PI3K }}$ tumors.

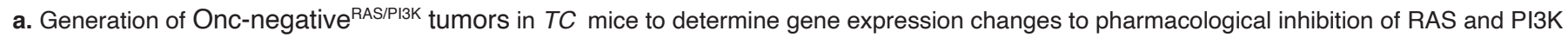
pathways. Indicated number of mice were treated with vehicle or combination of RMC-4550 and capivasertib 4.5 months after tumor initiation for three days. RNA-sequencing was performed on sorted Tomato ${ }^{\text {positive }}$ epithelial cells in tumors.

b. Volcano plots depicting a global overview of differential gene expression in Onc-negative ${ }^{\text {RAS/PIIK }}$ tumors in the absence and presence of treatment with RMC-4550 and capivasertib for three days as described above. Significant differential expression is defined as an absolute log2(Fold Change) > 1 and FDR $<0.01$. The numbers of significantly differentially expressed genes are indicated on the plot.

c-f. Comparison of RAS, PI3K-AKT, apoptosis, and G2M gene-set profiles estimated by single-sample Gene Set Enrichment Analysis (ssGSVA) in

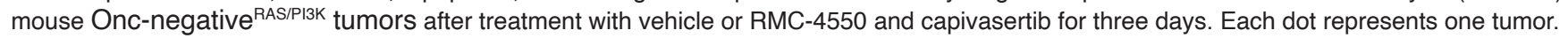
sSGSVA data points shown for vehicle-treated tumors are the same as Figure 13b, c as Nf1/Rasa1/Pten. The bar is mean. ns: non-significant, ${ }^{*} \mathrm{p}<0.05$ using Mann-Whitney U test. 
bioRxiv preprint doi: https://doi.org/10.1101/2021.10.20.464849; this version posted December 14, 2021. The copyright holder for this preprint (which was not certified by peer review) is the author/funder. All rights reserved. No reuse allowed without permission. Yousefi, Boross et al.

a

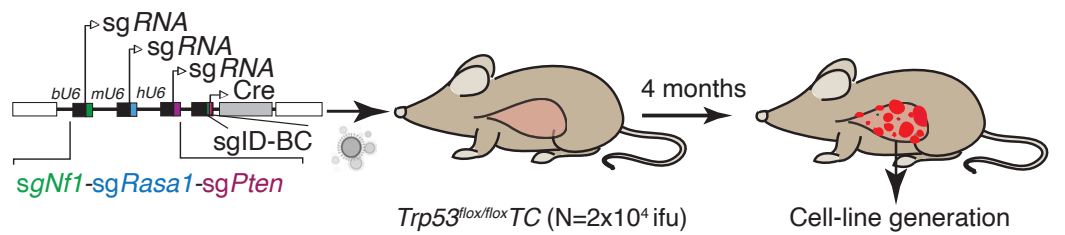

C

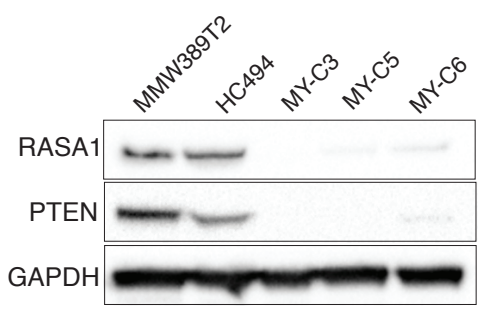

d

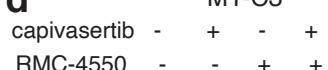

b

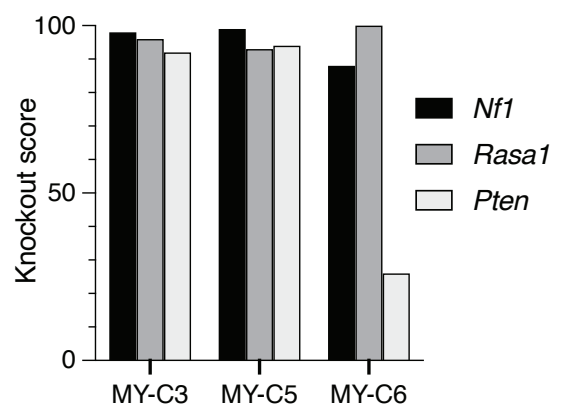

e

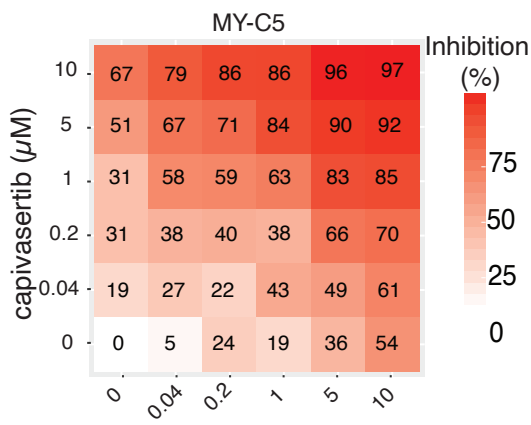

f

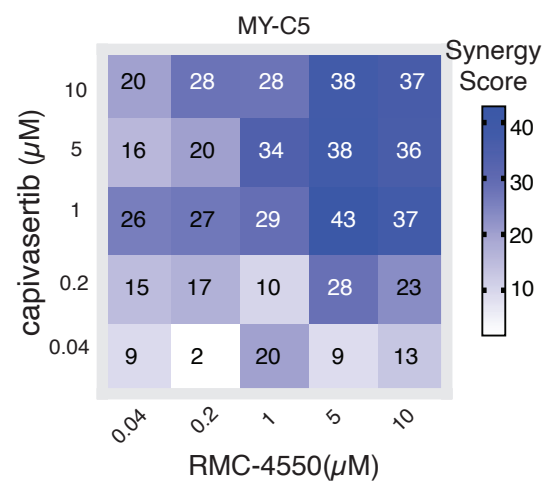

i

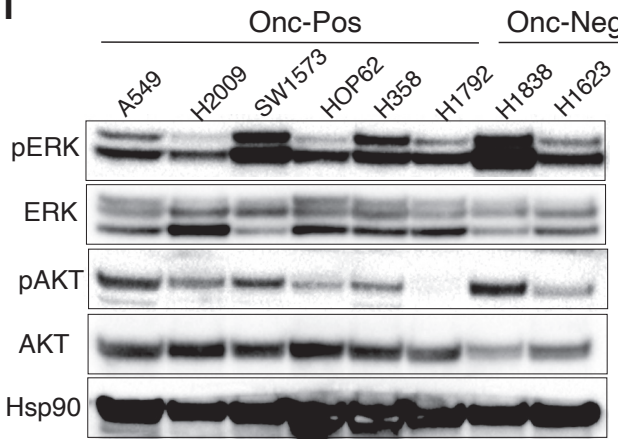

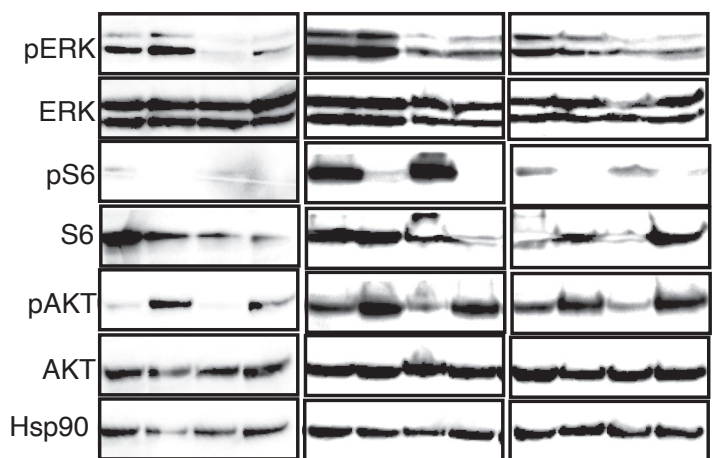

g

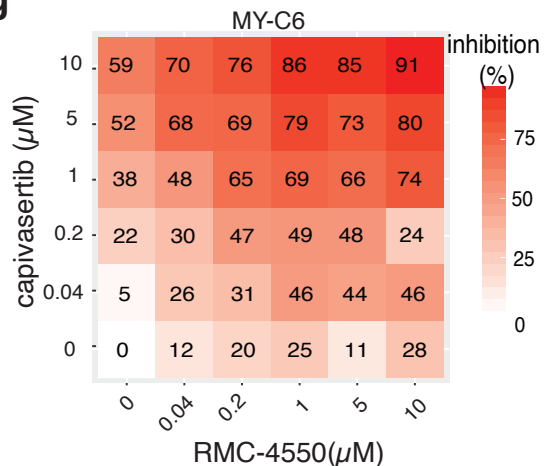

H1623

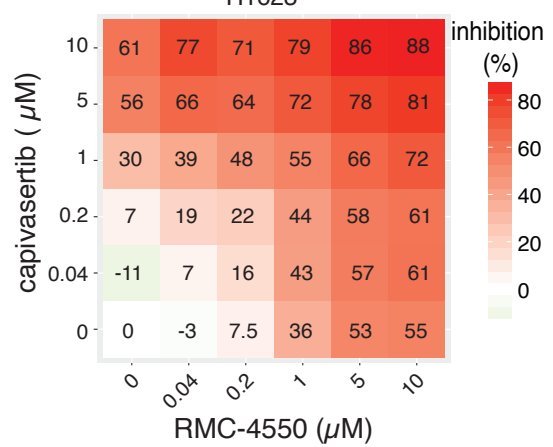

\section{h}

RMC-4550 $(\mu \mathrm{M})$

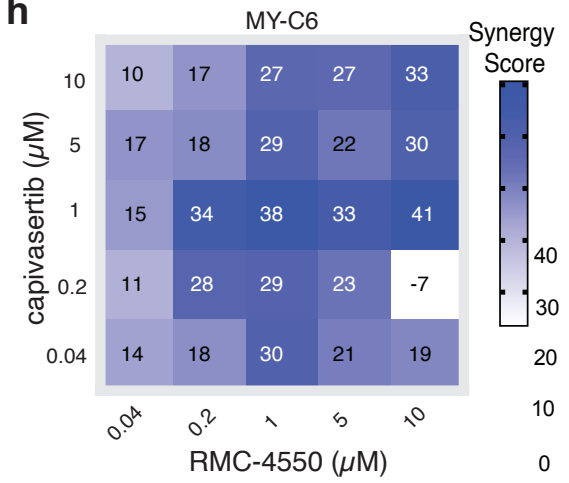

k

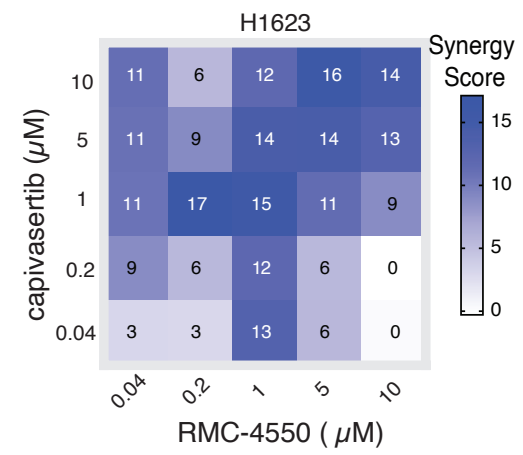

Figure S17. RMC-4550 synergizes with capivasertib to inhibit proliferation and induce cell death in Onc-negative ${ }^{\mathrm{RAS} / \mathrm{PI} 3 \mathrm{~K}}$ lung adenocarcinoma cell lines.

a. Cell line generation from Onc-negative ${ }^{\text {RAS/PISK }}$ tumors developed in Trp53 $3^{\text {floxflox }} ; T C$ mice.

b. Indel analysis of 3 distinct mouse oncogene-negative ${ }^{\mathrm{RAS} / \mathrm{PIKK}}$ cell lines described above. Regions targeted by sgNf1, sgRasa1, and sgPten were

PCR amplified and analyzed using Synthego ICE after sanger sequencing. Knockout score represents indels causing frameshift mutations.

c. Immunoblot of 2 murine oncogene-positive cell lines (MMW398T2 and HC494: Kras ${ }^{G 12 D}$ and Trp53 mutant and Nf1, Rasa1, and Pten wild type) and 3 murine Onc-negative ${ }^{\text {RAS/PIIK }}$ mouse cell lines (described above) to assess loss of RASA1 and PTEN in oncogene-negative cell lines.

d. Immunoblot of 3 distinct oncogene-negative cell-lines treated with 10uM of indicated drugs for 24 hours.

e,g. Drug dose-response matrix depicting \% growth inhibition after treatment with various doses of RMC- 4550 and capivasertib indicated on the plots.

The cell-line used for the generation of each matrix is noted on top of each heatmap.

$\mathbf{f}, \mathbf{h}$. Loewe's synergy score was calculated for each drug dose combination shown in $\mathbf{e}$ and $\mathbf{g}$. Synergy score indicates the percentage of inhibition beyond what is expected if there is no interaction between the drugs.

i. Immunoblot of 6 human oncogene-positive and 2 human oncogene-negative cell lines for markers of RAS and PI3K pathway activation.

j. Drug dose-response matrix depicting \% growth inhibition of $\mathrm{H} 1623$ human Onc-negative ${ }^{\text {RAS/PI3K }}$ cell line.

k. Loewe's synergy score calculated based on drug responses in Figure S17j. 


\section{METHODS}

Analysis of human lung adenocarcinoma datasets

Somatic mutation data (SNPs and indels, including silent mutations) for 513 TCGA lung

5 adenocarcinoma (LUAD) tumors were downloaded from the UCSC Xena Browser

6 (http://xena.ucsc.edu/) (Link 1 below). TCGA-LUAD clinical and exposure data were

7 downloaded from the GDC Data Portal (https://portal.gdc.cancer.gov/projects/TCGA-LUAD)

8 and the UCSC Xena Browser (Link 2 below). GISTIC2 thresholded copy number variation

9 (CNV) data were downloaded from the UCSC Xena Browser (Link 3 below). Amplifications

10 were defined as " 2 " and deletions as “-2". Genes with conflicting CNV values within a single

11 tumor were ignored. Fusion data were obtained from ${ }^{1}$. Fusion and CNV data were filtered to

12 include only data from the 513 samples within the somatic mutation set. Duplicate fusions were

13 collapsed into single fusions. MET-exon skipping data were taken from ${ }^{2}$. Curated survival data

14 from ${ }^{3}$ were downloaded from the UCSC Xena Browser (Link 4 below).

15 Links:

16 1. https://tcga.xenahubs.net/download/mc3/LUAD mc3.txt.gz

17 2. https://tcga.xenahubs.net/download/TCGA.LUAD.sampleMap/LUAD_clinicalMatrix

18 3. https://tcga.xenahubs.net/download/TCGA.LUAD.sampleMap/Gistic2_CopyNumber_Gi

19 stic2_all_thresholded.by_genes.gz

20 4. https://tcga.xenahubs.net/download/survival/LUAD survival.txt.gz

21 Data from AACR Project GENIE (hereinafter referred to as GENIE) v8 were downloaded

22 from $\underline{\text { https://www.synapse.org/\#!Synapse:syn22228642 }}{ }^{3}$, specifically: somatic mutations, copy

23 number alteration (CNA) data, fusion data, panel information (genomic_information.txt), and 
24 clinical data (both sample- and patient-level). All data were filtered to only include LUAD

25 tumors. A single tumor was kept for patients with multiple different tumor samples, with priority

26 for earlier sequenced samples and those from primary tumors. If tumor samples appeared

27 identical within the clinical meta-data, the related patient data were excluded.

29 Determination of oncogenes

To have a conservative estimate of the fraction of lung adenocarcinomas without known

31 oncogenic drivers (oncogene-negative tumors), we generated a list of oncogenes that included

32 any gene that met at least one of these criteria: 1) Genes that have hotspot mutations or specific

33 alterations where cancers or cancer cells with that mutation respond to therapies targeted to the

34 protein product of that mutant gene in patients, 2) The particular alteration in that gene can

35 generate lung adenocarcinoma in genetically-engineered mouse models, 3) The altered gene can

36 generate tumors in other tissues in genetically-engineered mouse models, and 4) Alteration of the

37 indicated gene can lead to the transformation of cells or predicts response to targeted therapies in

38 vitro. Additionally, we excluded genes if their supposed oncogenic alterations co-occur with

39 alterations in other proto-oncogenes (listed below) in more than $50 \%$ of cases.

\begin{tabular}{|l|l|l|l|l|l|l|}
\hline Gene & Oncogenic alterations & $\begin{array}{l}\text { Patients } \\
\text { respond to } \\
\text { oncogene } \\
\text { inhibition }\end{array}$ & $\begin{array}{l}\text { Sufficient to } \\
\text { generate lung } \\
\text { tumors in } \\
\text { GEMMs }\end{array}$ & $\begin{array}{l}\text { Oncogene in } \\
\text { GEMMs of } \\
\text { other tumor } \\
\text { types }\end{array}$ & $\begin{array}{l}\text { Cellular } \\
\text { transformation } \\
\text { and/or drug } \\
\text { response in } \\
\text { cultured cells }\end{array}$ & $\begin{array}{l}\text { Co- } \\
\text { occurring } \\
\text { with other } \\
\text { proto- } \\
\text { oncogenes } \\
\text { listed here }\end{array}$ \\
\hline KRAS & $\begin{array}{l}\text { Mutations at codons } \\
12,13 \text {, and 61 }\end{array}$ & 4,5 & $6-8$ & $7,9,10$ & & $4 \%$ \\
\hline$E G F R$ & $\begin{array}{l}\text { Diverse mutations and } \\
\text { deletions }\end{array}$ & 11,12 & $13-15$ & 16 & & $9 \%$ \\
\hline$B R A F$ & $\begin{array}{l}\text { Diverse mutations and } \\
\text { fusions }\end{array}$ & 17 & 18,19 & 20,21 & 22 & $18 \%$ \\
\hline$H R A S$ & $\begin{array}{l}\text { Mutations at codons 12, } \\
13, \text { and } 61\end{array}$ & & 23 & 24,25 & $13 \%$ \\
\hline
\end{tabular}




\begin{tabular}{|l|l|l|l|l|l|l|}
\hline NRAS & $\begin{array}{l}\text { Mutations at codons 12, } \\
13, \text { and 61 }\end{array}$ & & & 26,27 & & $14 \%$ \\
\hline MET & Exon skipping & 28,29 & 2 & & $2,30,31$ & $47 \%$ \\
\hline MEK1 & Diverse mutations & & & & 32 & $24 \%$ \\
\hline SOS1 & Diverse mutations & & & & 33 & $17 \%$ \\
\hline ALK & Translocations/fusions & 34 & 35,36 & 37 & 38 & $2 \%$ \\
\hline RET & Translocations/fusions & 39 & 40,41 & & & $10 \%$ \\
\hline ROS1 & Translocations/fusions & 42 & & 43,44 & & $4 \%$ \\
\hline NTRK1 & Translocations/fusions & 45 & & & 46 & $29 \%$ \\
\hline NRG1 & Translocations/fusions & 47 & & & & $0 \%$ \\
\hline AKT1 & Point mutation (E17K) & 48 & & & 49 & $50 \%$ \\
\hline RIT1 & Diverse mutations & & & & 50 & $19 \%$ \\
\hline ERBB2 & $\begin{array}{l}\text { Amplification, point } \\
\text { mutation, deletion }\end{array}$ & 51 & 52,53 & 54,55 & 56 & $68 \%$ \\
\hline PIK3CA & $\begin{array}{l}\text { Diverse mutations } \\
\text { n }\end{array}$ & & $\begin{array}{l}\text { Opposing } \\
\text { evidence } 57,58\end{array}$ & & & \\
\hline
\end{tabular}

42 Classification of mutations and tumors

44 were classified as within proto-oncogenes (described above) or not. Mutations within these

45 proto-oncogenes were classified as "accepted oncogenic" mutations if those alterations met at

46 least one of the criteria described above. Any tumor with one accepted oncogenic alteration was

47 classified as "oncogene-positive". Tumors with accepted oncogenic mutations in more than one

48 gene were classified as "multiple oncogenes mutated". Any tumor with alterations in a proto-

49 oncogene that was not considered an accepted oncogenic alteration based on the four criteria

50 above was classified as "oncogene-indeterminate". Thus, these tumors contain variants of

51 unknown significance (VUS) in proto-oncogenes ${ }^{59}$. The remaining tumors, without any

52 mutations in any proto-oncogene, were classified as "oncogene-negative".

53 Tumor type counts per database:

\begin{tabular}{|l|l|l|}
\hline & TCGA & GENIE \\
\hline Total & 513 & 9,099 \\
\hline Oncogene-negative & 91 & 1,645 \\
\hline Oncogene-positive & 283 & 6,041 \\
\hline Oncogene-indeterminant & 139 & 1,413 \\
\hline
\end{tabular}


Oncogene-positive tumors were further classified by the type of oncogenic mutation they

56 had (Figure 1a and S1a).

\section{Clinical characteristics}

We divided patients into males or females based on the sex reported by either TCGA or

60 GENIE, if provided. For TCGA, the arithmetic mean for age at diagnosis was computed and

61 reported with a standard error of the mean (SEM). Non-smokers were defined as having tobacco

62 smoking history values of 1 (see public ID 2181650 at https://cdebrowser.nci.nih.gov), while

63 smokers were defined as anything $>1$ (current or reformed smokers). The arithmetic mean pack-

64 years smoked for smokers, if reported, was described, along with SEM.

\section{Pan-cancer tumor suppressor genes}

We generated a list of tumor suppressor genes based on two previously published reports to compare the number of altered tumor suppressor genes in oncogene-negative tumors with

69 oncogene-positive and oncogene-indeterminate tumors ${ }^{60,61}$. We manually removed genes with

70 conflicting evidence as a tumor suppressor gene in LUAD. The final list of TSGs is in Table S1.

\section{Calculation of mutation frequencies and absolute number of genes mutated}

In general, mutation frequencies for a given gene were calculated as the number of

74 tumors with that gene mutated, divided by the number of tumors screened for mutations in that

75 gene (for TCGA: all tumors were screened for all genes, for GENIE: the panel sequencing

76 information was obtained from genomic_information.txt to determine which tumors were

77 screened for which genes). Mutation frequencies were calculated for point mutations (PM), 
78 insertion/deletions (indels), and deletions separately. Additionally, the frequency for a

79 combination of PMs, indels, and deletions was also calculated. The screened set of tumors in

80 GENIE for the latter included only those tumors which were screened for both PMs/indels as

81 well as CNVs for each gene. Reported in Figure S2b are oncogene-negative tumors with either

82 point mutations, indels, or deletions in the indicated gene. In Figure S2c-d, for each gene, a ratio

83 of enrichment of mutations in oncogene-negative over oncogene-positive tumors was calculated

84 as:

88 implemented by SciPy. For a given set of genes with at least a single tumor screened, the false

89 discovery rate (FDR) was calculated using the Benjamini-Hochberg method on the Fisher's

90 Exact $P$-values.

91 To measure the total number of genes mutated (Figure S1d), a gene was considered

92 mutated if it had at least one point mutation or indel. All these mutations in a tumor were

93 collated, and the number of the unique set of genes was counted as the total number of genes

94 mutated. For counting the number of individual tumor suppressors mutated (Figure S1e),

95 deletions were also included, and the list of pan-cancer tumor suppressors as defined above was

96 used. The Mann-Whitney U test was conducted on the number of respective genes mutated in

97 either oncogene-negative or oncogene-positive tumors.

98

\section{Survival Analysis}



performed to estimate probability curves for overall survival (OS) and disease-specific survival

102 (DSS). The logrank test was used to compare oncogene-negative and oncogene-positive tumors.

104 Gene and pathway alteration co-occurrences

105 For analysis of simultaneous pairwise alterations of $N F 1, R A S A 1$, or PTEN within

106 oncogene-negative tumors, we determined the number of tumors with no mutation in NF1,

107 RASA1, or PTEN, with mutation(s) in one gene, or mutations in two genes. Point mutations,

108 indels, and deletions in each gene were included. A tumor needed to have one or more mutations

109 in that gene to be considered mutated. For GENIE, only those tumors screened for both genes for

110 point mutations and indels (according to the panel information file) were investigated. For

111 TCGA, all oncogene-negative tumors were considered. A one-sided Fisher's exact test was

112 conducted to determine if there were more than the expected number of tumors with both genes

113 mutated.

114 Gene lists and their acceptable alterations (i.e., not known to be an oncogene alteration)

115 were generated as being in RAS or PI3K pathways ${ }^{60-80}$ (Table S6). We determined the list of all

116 tumors screened for each gene in each pathway for the respective type of mutation (point

117 mutation/indel, amplification, deletion, or fusion). For each alteration within each pathway, we

118 determined whether it could activate the corresponding pathway or not according to the above

119 list. A gene was considered mutated if it had at least one accepted mutation within it. A tumor

120 was considered mutated in a given pathway if it had at least one gene mutated in that pathway.

\section{Animal Studies}


The use of mice for the current study has been approved by Institutional Animal Care and

124 Use Committee at Stanford University, protocol number 26696. Kras ${ }^{L S L-G 12 D /+}$ (Jax \# 008179

125 (K)), R26 $6^{\text {LSL-tdTomato }}\left(\right.$ ai9) (Jax \# 007909 (T)), and H1 $1^{\text {LSL-Cas9 }}\left(\mathrm{Jax} \# 026816\right.$ (C)), Keap $1^{\text {flox }}$, Pten

126 flox (Jax \# 006440), Lkb1 $1^{\text {flox }}$ (Jax \# 014143), Nf1 flox (Jax \# 017640), and Trp53 flox (Jax \# 008462)

127 mice have been previously described ${ }^{6,81-87}$. All mice were on a C57BL/6:129 mixed background

128 except the mice used for derivation of oncogene-negative $N f 1$, Rasal, Pten, and Trp53 mutant

129 cell-lines, and some of the Trp53floxflox; $T C$ mice that were used for metastasis analysis (Figure

130 S12a), which were on a pure C57BL/6 background.

131

\section{Tumor initiation and selection of Lenti-sgRNA/Cre pools}

Tumors were initiated by intratracheal delivery of pooled or individual Lenti-sgRNA/Cre

134 vectors. Barcoded Lenti-sgRNA/Cre vectors within each viral pool are indicated in each figure.

135 Tumors were initiated with the indicated titers and allowed to develop tumors for between 3 and

13612 months after viral delivery, as indicated in each figure.

137 In Figure 1 and Figure S3, we transduced $N f 1^{f f f} ; T C, P t e n^{f / f} ; T C, T r p 53^{f / f} ; T C, L k b 1^{f f f} ; T C$,

138 Keapl ${ }^{f f f} ; T C, T C$, and $T$ mice with two pre-existing pools of barcoded Lenti-sgRNA/Cre vectors

139 that target $\sim 50$ putative tumor suppressor genes. These two pools have been previously used to

140 studied the effect of these putative tumor suppressor genes in $\mathrm{KRAS}^{\mathrm{G} 12 \mathrm{D}}$-driven lung tumors

141 (Lenti-sgTS15/Cre ${ }^{88,89}$ and Lenti-sgTS102/Cre ${ }^{90}$ ).

142 Lenti-sgTS15/Cre contained vectors targeting 11 tumor suppressors with one sgRNA per

143 gene in addition to four inert sgRNAs (Lenti-sgTS15/Cre) ${ }^{88,89}$. Lenti-sgTS102/Cre included

144 vectors targeting 48 tumor suppressors, including all five of the "core" tumor suppressors and 
145 most of the tumor suppressors targeted in Lenti-sgTS15/Cre with two or three sgRNAs per gene

146 in addition to five inert sgRNAs (102 sgRNA in total, Lenti-sgTS102/Cre) ${ }^{90}$ (See Table S1).

147 We determined the alteration frequency of many putative tumor suppressor genes,

148 including those targeted using our Lenti-sgTS15/Cre and Lenti-sgTS102/Cre pools, in oncogene-

149 positive and oncogene-negative tumors from TCGA and GENIE ${ }^{60,61}$. Alterations in only 17

150 tumor suppressor genes were significantly enriched in oncogene-negative tumors in GENIE and

151 most (12/17) were targeted by the Lenti-sgTS15/Cre and Lenti-sgTS102/Cre pools (Table S1).

152 We previously found that a small percent of lung tumors initiated with Lenti-sgRNA/Cre

153 vectors in other lung cancer models contained multiple sgRNAs, consistent with the transduction

154 of the initial cell with multiple Lenti-sgRNA/Cre vectors ${ }^{88,89}$. Thus, from tumor suppressor

155 genes that were found to be mutated in the largest tumors and expansions of experiment in

156 Figure 1, we selected 7 tumor suppressor genes that showed up in $\mathrm{Nf}^{\mathrm{fff}} ; \mathrm{TC}, \mathrm{Pten}^{\mathrm{fff}} ; \mathrm{TC}$,

$157 T r p 53^{f / f} ; T C$ mice in addition to 6 other tumor suppressor genes that showed significant effect in

158 at least one of these three backgrounds. For Studies in Figure 2 and Figure S7, we used higher

159 titers of Lenti-sgRNA/Cre vectors to increase the potential of finding higher-order interactions

160 that generate lung tumors. We found that simultaneous alterations of Nf1, Rasal, and Pten was

161 one of the most frequent co-occurring alterations in the largest tumors. Thus, we focused on

162 studying these three tumor suppressor alterations using Lenti-sgTripleTS8/Cre, Lenti-

163 sgTripleTS6/Cre, and Lenti-sgNf1-sgRasal-sgPten/Cre in the following figures.

164 The sgRNA sequences used in each experiment are summarized below. For a more

165 detailed description see Table S1:

\begin{tabular}{|l|l|}
\hline Pool & Pool composition \\
\hline Lenti-sgTS15/Cre & The exact pool used in ${ }^{88,89}$ \\
\hline
\end{tabular}




\begin{tabular}{|c|c|}
\hline Lenti-sgTS102/Cre & The exact pool used in 90 \\
\hline Lenti-sgTS14/Cre & 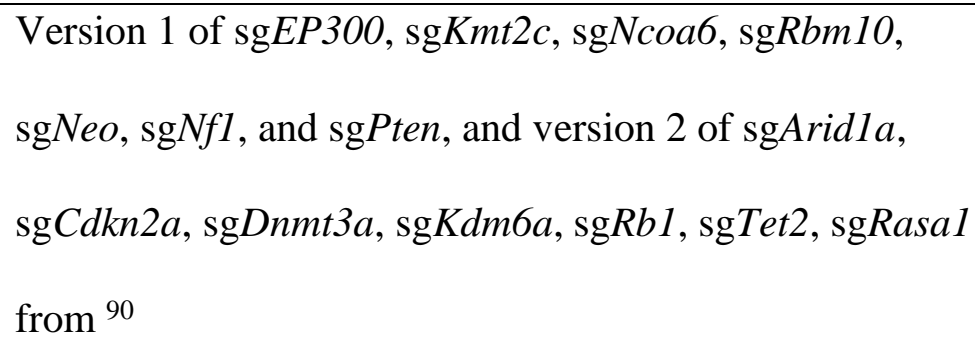 \\
\hline Lenti-sgTS11/Cre & $\begin{array}{l}\text { Lenti-sgTS14/Cre pool excluding sgNfl, sgRasal, and } \\
\text { sgPten }\end{array}$ \\
\hline Lenti-sgTripleTS8/Cre & 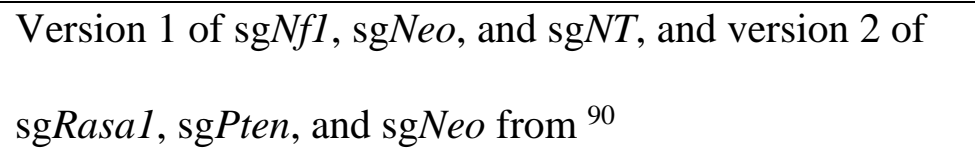 \\
\hline Lenti-sgTripleTS6/Cre & 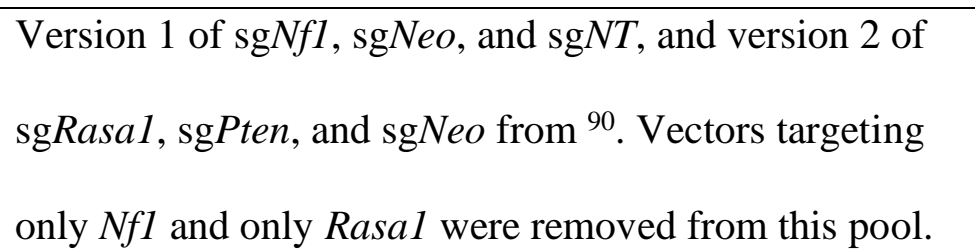 \\
\hline
\end{tabular}

\section{Lentiviral generation, barcoding, and packaging}

The sgRNA sequences, cloning, and barcoding of Lenti-sgRNA/Cre and Lenti-

170 TriplesgRNA/Cre vectors have been previously described ${ }^{88,90,91}$. To generate lentivirus, Lenti-

$171 \mathrm{sgRNA}$ Cre vectors were individually co-transfected into $293 \mathrm{~T}$ cells with pCMV-VSV-G

172 (Addgene \#8454) envelope plasmid and pCMV-dR8.2 dvpr (Addgene \#8455) packaging plasmid

173 using polyethylenimine. Supernatants were collected 36 and 48 hours after transfection, passed

174 through a $0.45 \mu \mathrm{m}$ syringe filter (Millipore SLHP033RB) to remove cells and cell debris,

175 concentrated by ultracentrifugation $\left(25,000 \mathrm{~g}\right.$ for 1.5 hours at $\left.4^{\circ} \mathrm{C}\right)$ and resuspended in $\mathrm{PBS}$

176 overnight. Each virus was titered against a standard of known titer using LSL-YFP Mouse 
177 Embryonic Fibroblasts (MEFs) (a gift from Dr. Alejandro Sweet-Cordero/UCSF). All lentiviral

178 vector aliquots were stored at $-80^{\circ} \mathrm{C}$ and were thawed and pooled immediately prior to delivery

179 to mice.

180

181 Tumor barcode sequencing and analysis

For DNA extraction from single dissected tumors to generate libraries for Tuba-seq,

183 targeted sequencing of selected oncogenes, and whole-exome sequencing, we used Qiagen

184 AllPrep DNA/RNA Micro kit. For Tuba-seq on bulk lungs, genomic DNA was isolated from

185 bulk tumor-bearing lung tissue from each mouse as previously described ${ }^{88}$. Briefly, benchmark

186 control cell lines were generated from LSL-YFP MEFs transduced by a barcoded Lenti-

187 sgNT3/Cre vector (NT3: an inert sgRNA with a unique sgRNA identifying barcode (sgID) and a

188 random barcode $(\mathrm{BC}))$ and purified by sorting $\mathrm{YFP}^{+}$cells using BD FACS Aria ${ }^{\mathrm{TM}}$ II Cell Sorter.

189 Three cell lines (100,000 to 500,000 cells each) were added to each mouse lung sample before

190 lysis to enable the calculation of the absolute number of neoplastic cells in each tumor from the

191 number of sgID-BC reads. Following homogenization and overnight protease K digestion,

192 genomic DNA was extracted from the lung lysates using standard phenol-chloroform and ethanol

193 precipitation methods. Subsequently, Q5 High-Fidelity 2x Master Mix (New England Biolabs,

194 M0494X) was used to amplify the sgID-BC region from $50 \mathrm{ng}$ of DNA from dissected tumors or

$19532 \mu \mathrm{g}$ of bulk lung genomic DNA. The unique dual-indexed primers used were Forward:

196 AATGATACGGCGACCACCGAGATCTACAC- 8 nucleotides for i5 index-

197 ACACTCTTTCCCTACACGACGCTCTTCCGATCT-6 to 9 random nucleotides for increasing

198 the diversity-GCGCACGTCTGCCGCGCTG and Reverse:

199 CAAGCAGAAGACGGCATACGAGAT-6 nucleotides for i7 index- 
GTGACTGGAGTTCAGACGTGTGCTCTTCCGATCT-9 to 6 random nucleotides for

201 increasing the diversity-CAGGTTCTTGCGAACCTCAT. The PCR products were purified with

202 Agencourt AMPure XP beads (Beckman Coulter, A63881) using a double size selection

203 protocol. The concentration and quality of the purified libraries were determined using the

204 Agilent High Sensitivity DNA kit (Agilent Technologies, 5067-4626) on the Agilent 2100

205 Bioanalyzer (Agilent Technologies, G2939BA). The libraries were pooled based on lung weights

206 to ensure even reading depth, cleaned up again using AMPure XP beads, and sequenced (read

207 length 2x150bp) on the Illumina HiSeq 2500 or NextSeq 500 platform (Admera Health

208 Biopharma Services).

210 Tuba-seq analysis of tumor barcode reads

211 The FASTQ files were parsed to identify the sgID and barcode (BC) for each read. Each

212 read is expected to contain an 8-nucleotide sgID region followed by a 30-nucleotide barcode

213 (BC) region (GCNNNNNTANNNNNGCNNNNNTANNNNNGC), and each of the 20 Ns

214 represents random nucleotides. The sgID region identifies the putative tumor suppressor gene

215 being targeted, for which we require a perfect match between the sequence in the forward read

216 and one of the forward sgIDs with known sequences. Note that all sgID sequences differ from

217 each other by at least three nucleotides. Therefore, the incorrect assignment of $\operatorname{sgID}$ due to PCR

218 or sequencing error is extremely unlikely. All cells generated from the clonal expansion of an

219 original cell transduced with a lentiviral vector carry the same BC sequence. To minimize the

220 effects of sequencing errors on calling the $\mathrm{BC}$, we require the forward and reverse reads to agree

221 completely within the 30-nucleotide sequence to be further processed. In our pipeline, any tumor

222 that is within a Hamming distance of two from a larger tumor is assigned as a "spurious tumor", 
223 which likely results from sequencing or PCR errors and the tumor is removed from subsequent

224 analysis. Reads with the same sgID and barcode are assigned to be the same tumor. The tumor

225 size (number of neoplastic cells) is calculated by normalizing the number of reads to the three

226 benchmarks "spike-in" cell lines added to each sample prior to lysis of the lung and DNA

227 extraction step. The median sequencing depth was $\sim 1$ read per 4.8 cells, and the minimum

228 sequencing depth is $\sim 1$ read per 16.5 cells. We have high statistical power in identifying tumors

229 with more than 200 cells, which was used as the minimum cell number cutoff for calling tumors.

230 A minimum cell number of 50 was used for calling expansions in Figures S5 and S6).

231 Minimizing the influence of GC amplification bias on tumor-size calling was done as previously

232 described ${ }^{88}$.

234 Measures of tumor size and growth

235 We used several metrics of tumor number, burden and size (see Supplemental Figure 4 in ${ }^{90}$ for 236 additional details on the calculation of these metric).

\begin{tabular}{|c|c|c|}
\hline $\begin{array}{l}\text { Measure of tumor } \\
\text { suppressor strength }\end{array}$ & Methodology & Description \\
\hline Surface tumor size & $\begin{array}{l}\text { visual } \\
\text { inspection }\end{array}$ & Tomato-positive expansions larger than $0.5 \mathrm{~mm}$ in diameter \\
\hline $\begin{array}{l}\text { Relative tumor size / } \\
\text { expansion size }\end{array}$ & Tuba-seq & $\begin{array}{l}\text { Tumor/expansion size at the indicated percentile was calculated } \\
\text { using tumors (clonal cell populations }>200 \text { cells) or expansions } \\
\text { (clonal cell populations }>50 \text { cells) merged from all mice and } \\
\text { normalized to the same percentile of sgInert tumors/expansions. }\end{array}$ \\
\hline Relative tumor burden & Tuba-seq & $\begin{array}{l}\text { Tumor burden was calculated as the sum of neoplastic cells per } \\
\text { mouse averaged over all mice and normalized to the tumor burden of } \\
\text { sgInert tumors. }\end{array}$ \\
\hline $\begin{array}{l}\text { Relative tumor } \\
\text { number }\end{array}$ & Tuba-seq & $\begin{array}{l}\text { Tumor numbers above a given size threshold (e.g., } 1000 \text { cells) were } \\
\text { determined by calculating the number of tumors above the threshold } \\
\text { per mouse averaged over all mice and normalized to the tumor } \\
\text { number of sgInert tumors. }\end{array}$ \\
\hline Relative frequency & Tuba-seq & $\begin{array}{l}\text { The relative frequency of each sgRNA was calculated in each } \\
\text { sample (one sample can contain multiple sgRNAs due to multiple } \\
\text { transduction or multiple tumors being present in the sample) and }\end{array}$ \\
\hline
\end{tabular}




\begin{tabular}{|l|l|l|} 
& $\begin{array}{l}\text { averaged for each sgRNA over all samples for a given mouse } \\
\text { genotype. }\end{array}$ \\
\hline $\begin{array}{l}\text { Frequency in large } \\
\text { tumors }\end{array}$ & Tuba-seq & $\begin{array}{l}\text { To find synergistic combinations in our data, we ranked all possible } \\
\text { combinations of targeted genes by their frequency of co-mutation in } \\
\text { the largest tumors. See Method section "Multiple transduction" for } \\
\text { how largest tumors and co-mutations of genes were defined. }\end{array}$ \\
\hline
\end{tabular}

240 sgRNA/Cre vector in the pool. When applicable, we used data on the number of tumors from $K T$

241 mice (which lack Cas9) to quantify the representation of each Lenti-sgRNA/Cre vectors in the

242 lentiviral pool. Therefore, when calculating tumor burden and tumor number metrics, we

243 normalized the metric to the effective titer based on data from $K T$ mice to account for the viral

244 titer differences among different Lenti-sgRNA/Cre vectors. Tumor/expansion size percentiles,

245 tumor burden, and tumor number were normalized to the values of the same metric for tumors

246 with inert sgRNAs, thus the expression "relative" is used.

247 For relative tumor/expansion size, relative tumor burden and relative tumor number,

248 confidence intervals and p- values were calculated by a nested bootstrap resampling approach to

249 account for variation in sizes of tumors of a given genotype both across and within mice. First,

250 tumors of each mouse were grouped, and these groups (mice) were resampled. Second, all

251 tumors of a given mouse resampling were bootstrapped on an individual basis $(10,000$

252 repetitions). For relative frequency, tumors were bootstrap resampled 10,000 times, and the

253 distribution of inert sgRNA frequencies was used to calculate p-values for enrichment of all

254 other sgRNAs. For "frequency in large tumors", a permutation test was used to calculate p-values

255 (see section Multiple transduction for details).

\section{Multiple transduction}


258 A fraction of lung tumors initiated with Lenti-sgRNA/Cre vectors contained multiple barcoded

259 Lenti-sgRNA/Cre vectors. If multiple barcodes (sgID-BCs) have unexpectedly similar read

260 counts (as shown in the example plots below), we suspect transduction of the initial cell with

261 multiple Lenti-sgRNA/Cre vectors.

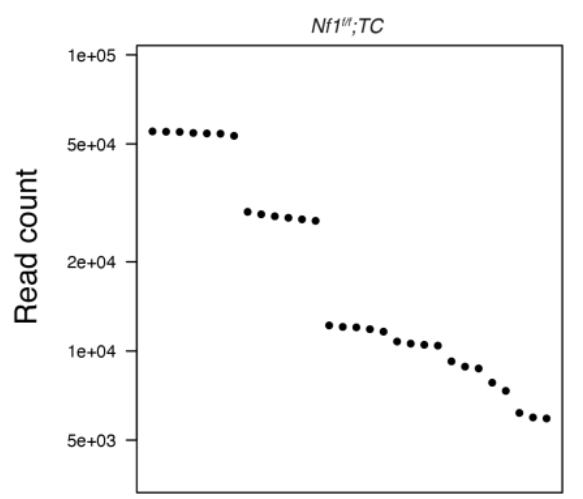

Example plots indicating strong evidence of transduction with multiple barcoded lentiviral vectors in the largest tumors

in each genotype. $30 \mathrm{sgID}$-BCs with the highest read counts from representative mouse samples are shown. Indicated genotypes of mice were transduced with Lenti-sgTS14/Cre pool. Each dot represents a sgID-BC, the y-axis shows read count, and the sgIDBCs are sorted on the x-axis by decreasing read counts. Groups of barcodes sgID-BCs that have very similar read counts likely represents a single clonal tumor initiated from a cell transduced with multiple barcoded Lenti-sgRNA/Cre vectors.

To capitalize on these multiple transductions as a way to find higher-order interactions between tumor suppressor genes, we developed a method to identify the combinations of sgRNA that appear to cooperate as potent drivers of tumor growth. Accurate identification of coinfected

274 genotypes with multiple tumor suppressor genes inactivated). For each sgID-BC, we listed all

275 other sgID-BCs from the same sample with read counts within $10 \%$ as possible multiple

276 transduction events. A tumor with multiple transductions can be most easily identified among the

277 largest tumors in each mouse as smaller tumors of similar sizes are too abundant. Multiple 
278 transductions that lead to synergistic combinatorial tumor suppressor alterations would confer a

279 growth advantage. Thus, synergistic combinatorial alterations of tumor suppressor genes would

280 be expected to be overrepresented among the largest tumors.

281 To have a dataset with a higher signal-to-noise ratio, we analyzed the largest tumors that

282 were co-infected with up to 6 Lenti-sgRNA/Cre vectors. With this method, for each tumor, we

283 assembled a list of genes that were possibly co-mutated. We then ranked all possible

284 combinations of genes by their frequency in the largest tumors (Figure 2f-g and S6c-h).

An inherent problem with this analysis is that the genotypes that increase tumor growth

286 will be overrepresented amongst the largest tumors even without multiple transductions and

287 specific synergistic interactions. To account for the different number of tumors with different

288 sgIDs, we performed a permutation test, where we control for the number of tumors of each

289 genotype but randomize the sizes of tumors by randomly matching the genotypes with tumor

290 sizes (10,000 repetitions). Synergistic tumor suppressor combinations will occur at significantly

291 higher than expected frequencies based on this permutation test (Figure $2 \mathbf{f}-\mathbf{g}$ and $\mathbf{S 6 c - h}$ ).

292 Reassuringly, while our analysis resulted in significant enrichment of complex genotypes based

293 on the permutation test, a control analysis performed on smaller tumors within the same mice

294 with high noise to signal ratio resulted in a loss of statistical significance, this shows that our

295 permutation test controls for the bias of different frequency of sgIDs among the tumors.

\section{Fitness landscape and adaptive paths}

To investigate the possible adaptive steps that can lead to the complex genotype of

299 coincident inactivation of $N f 1$, Rasal, and Pten, we first measured the fitness of all possible

300 combinations of $N f 1$, Rasal, and Pten mutations (Figure 3f and S10g). Relative (Malthusian) 
301 fitness was calculated based on the number of individuals (cells) at the end $\left(\mathrm{N}_{1}\right)$ and the

302 beginning of $\left(\mathrm{N}_{0}\right)$ of a time period ${ }^{92}$. For each genotype, the overall sum of neoplastic cells at

303 the end of the experiment $\left(\mathrm{N}_{1}\right)$ was calculated as the sum of cells from all tumors in each mouse.

304 As we use KT mice (which lack Cas9 and all sgRNAs have no effect) to approximate the

305 effective titer of our virus pool (see section Measures of tumor size and growth), the initial

306 number of cells transduced $\left(\mathrm{N}_{0}\right)$ was calculated from the number of tumors generated in control

$307 K T$ mice. Next, the relative fitness for genotype A compared to wild type (wt) was calculated as:

$$
\frac{\log 2 \frac{N_{1, A}}{N_{0, A}}}{\log 2 \frac{N_{1, w t}}{N_{0, w t}}}
$$

Fitness values relative to wild type are displayed as nodes on the adaptive landscape

(Figure 3f and S10g), where genotypes one mutation away from each other are connected by

311 arrows that represent mutations. In the case of the Nfl;Rasal;Pten triple mutant state, six

312 adaptive paths can lead from wild type to that triple mutant genotype (Figure 3f and $\mathbf{S 1 0 g}$ ).

313 Arrows are shown if the mutation increases the fitness. In figure $\mathbf{3 f}$ and $\mathbf{S 1 0 g}$ all arrows are

314 shown since all mutations increase fitness.

Next, we set out to approximate the relative probabilities of different adaptive paths

316 leading from wild type to the triple mutant genotype with a simple population genetic model. In

317 the model, cell populations start from the wild-type genotype, and they can acquire any of the

318 three mutations present in the triple genotype. In the population of cells, a mutation can arise and

319 then change in frequency until one of two outcomes happens: (i) the frequency of the mutation

320 drops to zero, and the mutation is lost from the population or (ii) the frequency of the mutation

321 reaches 1 , when it is present in all the cells and hence is fixed in the population. When a

322 mutation fixes in a population, we consider the genotype of the population to change and that 
323 constitutes a "step" on the fitness landscape. We assume a "strong selection weak mutation"

324 regime, where there is no more than one mutation simultaneously present with a frequency less

325 than 1 . We also assume that mutations appear randomly and with equal probabilities. Mutations

326 can appear and get lost multiple times in a population, and as long as populations have at least

327 one mutation that increases fitness, one of those mutations will fix in the population eventually.

328 With the model we are estimating the probability of each adaptive step given that the

329 population starts from the wild-type state. Therefore, the probability of each adaptive step will be

330 influenced by the probabilities of previous step(s) and the sum of probabilities of adaptive steps

331 originating from a given genotype must equal the sum of probabilities of all adaptive steps

332 terminating in the given genotype. If there are multiple adaptive steps originating from the same

333 genotype, they will have probabilities proportional to the fixation probabilities of their respective

334 mutations. The fixation probability of a mutation is proportional to its selective advantage

335 ( $\left.\frac{\text { fitness after mutation }}{\text { fitness before mutation }}-1\right)^{93}$. As an example, if there are two adaptive steps originating from a

336 genotype with fitness 1.00 , one terminating in a genotype with fitness 1.1 , the other in a

337 genotype with fitness 1.2 , then they have $10 \%$ and $20 \%$ selective advantage, respectively.

338 Therefore, one adaptive step will happen half as likely as the other, as the selective advantages

339 and therefore the relative fixation probabilities are in a ratio of $1: 2$.

\section{Targeted sequencing of oncogenic loci for potential spontaneous oncogenic mutation}

342 To determine whether the tumors that develop contained spontaneous oncogenic

343 mutations, we performed Sanger Sequencing and Illumina sequencing (HiSeq 2500 platform;

344 read length 2x150 bp, Admera Health Biopharma Services) on select regions of Kras, Egfr, Braf,

345 and Nras (the 4 frequently mutated oncogenes in lung adenocarcinoma). 
PCR products were obtained through amplification with primers listed below on DNA

347 extracted from dissected tumors (Table S2) and cleaned up using ExoSAP (ThermoFisher

348 Scientific, Cat\# 78-201) treatment before Sanger.

\begin{tabular}{|c|c|}
\hline $\begin{array}{l}\text { Oncogene and } \\
\text { associated codon }\end{array}$ & Primer \\
\hline $\begin{array}{l}\text { Kras Codon } 12+13 \\
\text { Forward }\end{array}$ & $\begin{array}{l}\text { ACACTCTTTCCCTACACGACGCTCTTCCGATCTNN } \\
\text { NNNNTTATTTTTATTGTAAGGCCTGCT }\end{array}$ \\
\hline $\begin{array}{l}\text { Kras Codon } 12+13 \\
\text { Reverse }\end{array}$ & $\begin{array}{l}\text { GTGACTGGAGTTCAGACGTGTGCTCTTCCGATCTN } \\
\text { NNNNNNTTACAAGCGCACGCAGA }\end{array}$ \\
\hline $\begin{array}{l}\text { Kras Codon } 61 \\
\text { Forward }\end{array}$ & $\begin{array}{l}\text { ACACTCTTTCCCTACACGACGCTCTTCCGATCTNN } \\
\text { NNNNNNCCTGTCTCTTGGATATTCTCGAC }\end{array}$ \\
\hline Kras Codon 61 Reverse & $\begin{array}{l}\text { GTGACTGGAGTTCAGACGTGTGCTCTTCCGATCTN } \\
\text { NNNNNNNNCAGTTCTCATGTACTGGTCCCT }\end{array}$ \\
\hline $\begin{array}{l}\text { Egfr Codon } 721 \\
\text { Forward }\end{array}$ & $\begin{array}{l}\text { ACACTCTTTCCCTACACGACGCTCTTCCGATCTNN } \\
\text { NNNNNNCCAGCGGAGAAGCTCCAAAC }\end{array}$ \\
\hline $\begin{array}{l}\text { Egfr Codon } 721 \\
\text { Reverse }\end{array}$ & $\begin{array}{l}\text { GTGACTGGAGTTCAGACGTGTGCTCTTCCGATCTN } \\
\text { NNNNNNNNATACACTGTGCCAAATGCTCCC }\end{array}$ \\
\hline $\begin{array}{l}\text { Egfr Codon 734-756 } \\
\text { Forward }\end{array}$ & $\begin{array}{l}\text { ACACTCTTTCCCTACACGACGCTCTTCCGATCTNN } \\
\text { NNNNTCTTCTTAATCTCAGGGTCTCTGG }\end{array}$ \\
\hline $\begin{array}{l}\text { Egfr Codon 734-756 } \\
\text { Reverse }\end{array}$ & $\begin{array}{l}\text { GTGACTGGAGTTCAGACGTGTGCTCTTCCGATCTN } \\
\text { NNNNNNCACGTCAAGGATTTCTTTGTTGGC }\end{array}$ \\
\hline
\end{tabular}




\begin{tabular}{|c|c|}
\hline $\begin{array}{l}\text { Egfr Codon 764-793 } \\
\text { Forward }\end{array}$ & $\begin{array}{l}\text { ACACTCTTTCCCTACACGACGCTCTTCCGATCTNN } \\
\text { NNNNNNTTACCCAGAAAGGGATATGCGTG }\end{array}$ \\
\hline Egfr Codon 764-793 & GTGACTGGAGTTCAGACGTGTGCTCTTCCGATCTN \\
\hline Reverse & NNNNNNNNGGCAACCGTAGGGCATGAG \\
\hline Egfr Codon $860+863$ & ACACTCTTTCCCTACACGACGCTCTTCCGATCTNN \\
\hline Forward & NNNNGTGAAGACACCACAGCATGTCAAG \\
\hline Egfr Codon $860+863$ & GTGACTGGAGTTCAGACGTGTGCTCTTCCGATCTN \\
\hline Reverse & NNNNNNGCTTCCTGATCTACTCCCAGGAC \\
\hline Braf Codon 503-509 & ACACTCTTTCCCTACACGACGCTCTTCCGATCTNN \\
\hline Forward & NNNNNNGACTGGGAGATTCCTGATGGAC \\
\hline Braf Codon 503-509 & GTGACTGGAGTTCAGACGTGTGCTCTTCCGATCTN \\
\hline Reverse & NNNNNNNNcgtgttatacataccatgtcccac \\
\hline Braf Codon 637 & ACACTCTTTCCCTACACGACGCTCTTCCGATCTNN \\
\hline Forward & NNNNGACCTCACGGTAAAAATAGGTGAC \\
\hline Braf Codon 637 & GTGACTGGAGTTCAGACGTGTGCTCTTCCGATCTN \\
\hline Reverse & NNNNNNAACTGTTCAAACTGATGGGACC \\
\hline Nras Codon $12+13$ & ACACTCTTTCCCTACACGACGCTCTTCCGATCTNN \\
\hline Forward & NNNNNNTTCTACAGGTTTTTGCTGGTGTG \\
\hline Nras Codon $12+13$ & GTGACTGGAGTTCAGACGTGTGCTCTTCCGATCTN \\
\hline Reverse & NNNNNNNNGATTAGCTGGATCGTCAAGGC \\
\hline
\end{tabular}




\begin{tabular}{|l|l|}
\hline Nras Codon 61 & ACACTCTTTCCCTACACGACGCTCTTCCGATCTNN \\
Forward & NNNNCGAAAGCAAGTGGTGATTGATGG \\
\hline Nras Codon 61 Reverse & NNNNNNAAATACACAGAGGAACCCTTCG \\
\hline
\end{tabular}

350 Illumina sequencing was performed on pools of amplicons. The libraries were pooled

351 based on band intensity to ensure even read depth and cleaned up using Sera-Mag Select beads

352 (Thermo Fisher Scientific, Cat\# 09-928-107) before undergoing a second round of PCR to attach

353 the sequencing adaptors needed for the HiSeq platform. Second round PCR products were then

354 purified with Sera-Mag Select beads before sequencing.

355 N's represent i5 and i7 indices.

\begin{tabular}{|l|l|}
\hline P5 adapter with i5 & AATGATACGGCGACCACCGAGATCTACACNNNNN \\
Index & NNNacactctttcctacacgac \\
\hline P7 adapter with i7 & CAAGCAGAAGACGGCATACGAGATNNNNNNgtgact \\
Index & ggagttcagacgtg \\
\hline
\end{tabular}

Analysis of targeted DNA-sequencing of Kras, Egfr, Braf, and Nras oncogenic loci 
364 with $90 \%$ of regions having a coverage over 275 reads in all samples. Variant calls made and

365 filtered by GATK Mutect2 function were annotated with Ensembl Variant Effect Predictor ${ }^{95}$.

366 Pick-allele-gene option was used to filter results on the most relevant transcript for each

367 variation. We filtered the results for the known oncogenic codons listed above and variants with

368 a minimum of $5 \%$ allele frequency.

Whole exome sequencing

DNA was extracted from 4 individual tumors from $T C$ mice transduced with Lenti-sgNfl-

372 sgRasal-sgPten, three months after tumor initiation, using Qiagen AllPrep DNA/RNA Micro kit.

373 Whole-exome sequencing library preparation was performed by Admera Health using SureSelect

374 XT Mouse All Exon Kit (Agilent).

375 Sequenced reads on autosomes were analyzed using Genome Analysis Toolkit (GATK,

376 Broad Institute ${ }^{94}$ ) "Somatic short variant discovery" best practices pipeline for tumor samples.

377 Mean coverage of 50-72 reads was achieved for all samples, with $90 \%$ of regions having

378 coverage over 20 reads in all samples. Variant calls made and filtered by GATK Mutect2

379 function and were annotated with Ensembl Variant Effect Predictor (VEP ${ }^{95}$ ). The pick-allele-

380 gene option was used to filter results on the most relevant transcript for each variant. The same

381 exact variants appearing in multiple tumor samples were flagged as germline variant and were

382 removed. We filtered the results for protein-coding variation, variants with a minimum of $5 \%$

383 allele frequency, and removed variations in the olfactory OLFR gene family that are likely

384 germline variations. 
387 Indel analysis was performed to confirm that insertion and deletions (indels) were generated at

388 the targeted loci as follows: gDNA was isolated from at oncogene-negative mouse cell lines or

389 FACS-sorted Tomato ${ }^{\text {positive }}$ cancer cells using either the AllPrep DNA/RNA(Qiagen) or the

390 DNeasy Blood and Tissue Kit. PCR primers were designed to amplify sgRNA-targeted loci,

391 resulting in 500 to $1000 \mathrm{bp}$ amplicons specific to each locus. Amplicons were purified using

392 PCR purification kit (Qiagen) and sequenced by Sanger sequencing. Cutting efficiency was

393 determined by ICE analysis (https://ice.synthego.com/\#/)

\begin{tabular}{|l|l|}
\hline Targeted gene & Primer \\
\hline Nfl-Amplification Forward primer & GCAATTTTGGGGGAACGCCT \\
\hline Nfl-Amplification Reverse primer & AAAACCAAGAGAGGTCAGAGCC \\
\hline Nfl-Sequencing primer & CAGCGATTCTAAAATACCAATGC \\
\hline Rasal-Amplification Forward primer & GGAGCACGGTATGTGTCGTT \\
\hline Rasal-Amplification Reverse primer & TCCTCTTTAGCGTAGCCAGGAA \\
\hline Rasal-Sequencing primer & TTGGTGAAAGCGACGTCTC \\
\hline Pten-Amplification Forward primer & TGAATACACAGTGGCCTTTGCTT \\
\hline Pten-Amplification Reverse primer & CAGAGACTGCATCTGGTGGTT \\
\hline Pten-Sequencing primer & CATTGGGTTAGCTTTCTTAACC \\
\hline
\end{tabular}


Lung lobes were inflated with $4 \%$ formalin and fixed for 24 hours, stored in $70 \%$ ethanol, paraffin-embedded, and sectioned. $4 \mu \mathrm{m}$ thick sections were used for Hematoxylin and Eosin

398 (H\&E) staining and immunohistochemistry (IHC).

Primary antibodies used for IHC were anti-RFP (Rockland, 600-401-379), anti-

401 Technology, 13109), anti-phospho-S6 (Cell Signaling Technology, 4858), anti-PTEN (Cell

402 Signaling Technology, 9559), anti-phospho-ERK (Cell Signaling Technology, 4370), anti-

403 phospho-AKT (Thermo Fisher Scientific, 44-621G), and anti-HMHGA2 (Biocheck, 59170AP).

404 IHC was performed using Avidin/Biotin Blocking Kit (Vector Laboratories, SP-2001), Avidin-

405 Biotin Complex kit (Vector Laboratories, PK-4001), and DAB Peroxidase Substrate Kit (Vector

406 Laboratories, SK-4100) following standard protocols.

408 converted from pixels to $\mathrm{mm}^{2}$ via a ruler. To quantify the positivity of phospho-ERK and

409 phospho-AKT stained slides, H-scores were calculated using Qupath. The H-score is determined

410 by adding the results of multiplication of the percentage of cells with staining intensity ordinal

411 value (scored from 0 for "no signal" to 3 for "strong signal") with possible values ranging from 0

412 to $300^{96}$. To normalize potential variations between different rounds of immunohistochemistry,

413 one patient sample was included and stained for both pERK and pAKT in all rounds of staining

414 as a control.

Immunoblotting

$3 \times 10^{5}$ cells were seeded into 6-well plates and allowed to adhere overnight in regular

418 growth media and cultured in the presence or absence of $10 \mu \mathrm{M}$ of Capivasertib, RMC-4550, or 
419 a combination of both drugs. After 24 hours, the protein was extracted using RIPA lysis buffer

420 (Thermo Fisher Scientific, 89900) and proteinase/phosphatase inhibitor cocktail (Thermo Fisher

421 Scientific, 78442). Protein concentration was measured using BCA protein assay kit (Thermo

422 Fisher Scientific, 23250). Proteins (30 $\mu \mathrm{g}$ from each sample) were separated by SDS-PAGE and

423 immunoblotted and transferred to polyvinyl difluoride (PVDF) membranes (BioRad, 162-0177)

424 according to standard protocols. Membranes were immunoblotted with antibodies against

425 phosphor-ERK (Cell Signaling Technology, 4370), ERK (Cell Signaling Technology, 9102),

426 phosphor-AKT (Thermo Fisher Scientific, 44-621G), AKT (Cell Signaling Technology, 4691),

427 phospho-S6 (Cell Signaling Technology, 4858), S6 (Cell Signaling Technology, 2217), anti-

428 RASA1 (Abcam, ab2922), anti-PTEN (Cell Signaling Technology, 9559), and HSP90 (BD

429 Bioscience, 610418). Immunoblots were developed using Supersignal® West Dura Extended

430 Duration Chemiluminescent Substrate (Thermo Fisher Scientific, 37071). Initially, the

431 membranes were immunoblotted against non-phosphorylated targets, and after stripping these

432 antibodies using Western Blot Stripping Buffer (Thermo Fisher Scientific, 46430), they were

433 immunoblotted against phosphorylated antibodies. Developing the signal was done using Dura

434 Extended Duration Chemiluminescent Substrate (Thermo Fisher Scientific, 37071). All

435 immunoblots were performed at least three times independently.

\section{Cell Lines and Reagents}

438 Mouse oncogene-negative cell lines were generated from tumors initiated in

439 Trp53floxflox; $T C$ BL6 mice four months after transduction with Lenti-sgNf1-sgRasal-sgPten/Cre.

440 After dissociation of tumors (described below), cells were cultured in DMEM supplemented with

441 10\% FBS, 1\% penicillin/streptomycin (Gibco), and 0.1\% Amphotericin (Life Technologies). 
442 HC494 and MMW389T2 (Kras ${ }^{G 12 D}$ and Trp53 mutant) lung adenocarcinoma cells were

443 previously generated in the Winslow Lab. Human oncogene-negative cell lines (NCI-H1838,

444 NCI-H1623) and oncogene-positive cell lines (A549, H2009, NCI-H2009, SW1573, HOP62,

445 NCI-H358, NCI-H1792) were purchased from ATCC and cultured in RPMI supplemented with

$4465 \% \mathrm{FBS}, 1 \%$ penicillin/streptomycin (Gibco), and 0.1\% Amphotericin (Life Technologies). We

447 performed mycoplasma testing using MycoAlert ${ }^{\mathrm{TM}}$ Mycoplasma Detection Kit (Lonza). Cell

448 were maintained at $37^{\circ} \mathrm{C}$ in a humidified incubator at $5 \% \mathrm{CO}_{2}$. Mutations in components of RAS

449 and PI3K pathways of NCI-H1838, NCI-H1623 (based on Table S6) are indicated in the table

450 below (extracted from DepMap):

\begin{tabular}{|c|c|c|c|}
\hline Cell line & Pathway & Gene & Protein Change \\
\hline NCI-H1838 & RAS & NF1 & p.N184fs \\
\hline NCI-H1838 & RAS & IQGAP2 & p.P780L \\
\hline NCI-H1623 & RAS & FGFR2 & p.A355S \\
\hline NCI-H1623 & RAS & ERF & p.G255C \\
\hline NCI-H1623 & RAS & RASA1 & p.A47fs \\
\hline
\end{tabular}

\section{Clonogenic, apoptosis, and proliferation assays}

For clonogenic assays, mouse cells were seeded in triplicate into 24-well plates (4000

454 cells per well) and allowed to adhere overnight in regular growth media. Cells were then cultured

455 in the absence or presence of the drug as indicated on each figure panel in complete media for 4

456 days. Growth media with or without drugs was replaced every 2 days. The remaining cells were

457 stained with $0.5 \%$ crystal violet in $20 \%$ methanol and photographed using a digital scanner. 
458 Relative growth was quantified by densitometry after extracting crystal violet from the stained 459 cells using $100 \%$ methanol ${ }^{97}$.

460 Clonogenic assay of human oncogene-negative lung adenocarcinoma cell lines were done 461 in spheroids ${ }^{98} .400-5000$ cells/well were seeded in round bottom ultra-low attachment 96-well 462 plates (Corning) in growth media and incubated for 72 hours at $37^{\circ} \mathrm{C}$ in $5 \% \mathrm{CO} 2$. Spheroid 463 formation was confirmed visually, and spheroids were treated in triplicate with dilutions of 464 RMC-4550 and capivasertib in complete growth media. Following drug exposure for five days, 465 cell viability in spheroids was determined using the CellTiter-Glo 3D assay kit (Promega), 466 following the manufacturer's instructions. Luminescence was read in a Plate Reader. Assay data 467 was normalized to DMSO values.

468 Drug synergism was analyzed using SynergyFinder (https://synergyfinder.fimm.fi) web 469 application ${ }^{99}$. The degree of combination synergy, or antagonism, was quantified by comparing 470 the observed drug combination response against the expected response, calculated using Loewe's 471 model that assumes no interaction between drugs ${ }^{100}$.

472 For apoptosis and proliferation assays, $3 \times 10^{5}$ cells were seeded into 6-well plates, and 473 allowed to adhere overnight in regular growth media, and cultured in the presence or absence of $47410 \mu \mathrm{M}$ of Capivasertib, RMC-4550, or a combination of both drugs. After 24 hours, apoptosis 475 and cell proliferation were determined through staining with Fixable Viability Dye eFluor ${ }^{\mathrm{TM}} 450$ 476 (Thermo Fisher Scientific, 65-0863-14), cleaved caspase 3 Antibody (Cell Signaling 477 Technology, 9669), and Click-iT ${ }^{\mathrm{TM}}$ EdU Alexa Fluor ${ }^{\mathrm{TM}} 647$ Flow Cytometry Assay Kit (Thermo 478 Fisher Scientific, C-10424) according to the manufacturer's instructions. Data were acquired 479 using a BD LSR II Flow Cytometer. All experiments were performed independently two times 480 on 3 different cell lines. 


\section{In vivo drug response studies}

For drug efficacy studies in autochthonous mouse models, $T C$ mice (8-12 weeks old)

484 were divided into 4 groups randomly 3.5 months after tumour initiation. They received the

485 vehicle, capivasertib (100 mg/kg, MedChemExpress), RMC-4550 (30 mg/kg,

486 MedChemExpress), or a combination of both dissolved in 10\% DMSO, 40\% PEG, 5\% Tween

487 80, and 45\% PBS through a gavage needle. Mice were treated daily with drugs for eight days,

488 and the treatment was stopped for two days for recovery, and it continued for two more days

489 before the tissue harvest. The last two doses of combination therapy were half of the initial

490 doses.

491

492

Tumor dissociation, cell sorting, and RNA-sequencing

Primary tumors were dissociated using collagenase IV, dispase, and trypsin at $37{ }^{\circ} \mathrm{C}$ for

$49430 \mathrm{~min}$. After dissociation, the samples remained continually on ice, were in contact with ice-

495 cold solutions, and were in the presence of $2 \mathrm{mM}$ EDTA and $1 \mathrm{U} / \mathrm{ml}$ DNase to prevent

496 aggregation. Cells were stained with antibodies to CD45 (BioLegened, 103112), CD31

497 (BioLegend, 303116), F4/80 (BioLegend, 123116), and Ter119 (BioLegend, 116212) to exclude

498 hematopoietic and endothelial cells (lineage-positive ( $\left.\operatorname{Lin}^{+}\right)$cells). DAPI was used to exclude

499 dead cells. FACS Aria sorters (BD Biosciences) were used for cell sorting.

500 RNA was purified using RNA/DNA All Prep kit (Qiagen, 80284). RNA quality of each tumor

501 sample was assessed using the RNA6000 PicoAssay for the Agilent 2100 Bioanalyzer as per the

502 manufacturer's recommendation. 4.4 ng total RNA per sample was used for cDNA synthesis and

503 library preparation using Trio RNA-Seq, Mouse rRNA kit (Tecan, 0507-32), according to the 
504 manufacturer's instructions. The purified cDNA library products were evaluated using the

505 Agilent bioanalyzer and sequenced on NextSeq High Output 1x75 (Admera Health Biopharma

506 Services).

507

Analysis of mouse model-derived RNA-seq datasets

Paired-end RNA-seq reads were aligned to the mm10 mouse genome using STAR

510 (v2.6.1d) 2-pass mapping and estimates of transcript abundance were obtained using RSEM

511 (v1.2.30) ${ }^{101,102}$. The differentially expressed genes between different tumor genotypes and

512 treatment groups were called by DESeq2 using transcript abundance estimates via tximport ${ }^{103 \text {, }}$

$513{ }^{104}$. The DESeq2-calculated fold changes were used to generate ranked gene lists for input into

514 GSEA ${ }^{105}$.

515 The upregulated genes with absolute $\log 2$ fold change greater than 1 and a false

516 discovery rate less than 0.05 in the comparison of $N f 1$, Rasal, and Pten mutant oncogene-

517 negative tumors with $\mathrm{Kras}^{\mathrm{G} 12 \mathrm{D}}$-driven tumors (KTC+sgInert and KTC+sgPten)

518 were compiled into a signature reflecting the oncogene-negative adenocarcinoma state. This gene

519 signature was utilized in the analysis of human oncogene-positive and oncogene-negative

520 tumors. Scaled estimates of transcript abundance for TCGA LUAD samples were obtained from

521 the GDC data portal (gdc-portal.nci.nih.gov). Each expression profile was then scored on the

522 basis of the mouse-derived gene signature using single-sample GSEA within the Gene Set

523 Variation Analysis (GSVA) package ${ }^{106}$.

\section{Data availability}


526 Tuba-seq barcode sequencing and RNA-seq data have been deposited in NCBI's Gene

527 Expression Omnibus (https://www.ncbi.nlm.nih.gov/geo/) and are accessible through GEO

528 Series accession number GSE174393. Whole exome sequencing data generated in our study are

529 publicly available in SRA-NCBI (www.ncbi.nlm.nih.gov/sra), under BioProject accession

530 number PRJNA769722.

\section{Acknowledgments}

533 The results in Figures 1 and 4 are in part based upon data generated by the TCGA Research

534 Network (https://www.cancer.gov/tcga) and Genomics Evidence Neoplasia Information

535 Exchange (GENIE).

\section{References:}

1. Gao, Q. et al. Driver Fusions and Their Implications in the Development and Treatment of Human Cancers. Cell Rep 23, 227-238 e223 (2018).

2. Lu, X. et al. MET Exon 14 Mutation Encodes an Actionable Therapeutic Target in Lung Adenocarcinoma. Cancer Res 77, 4498-4505 (2017).

3. Liu, J. et al. An Integrated TCGA Pan-Cancer Clinical Data Resource to Drive High-Quality Survival Outcome Analytics. Cell 173, 400-416 e411 (2018).

4. Hallin, J. et al. The KRAS(G12C) Inhibitor MRTX849 Provides Insight toward Therapeutic Susceptibility of KRAS-Mutant Cancers in Mouse Models and Patients. Cancer Discov 10, 54-71 (2020).

5. Canon, J. et al. The clinical KRAS(G12C) inhibitor AMG 510 drives anti-tumour immunity. Nature 575, 217-223 (2019).

6. Jackson, E.L. et al. Analysis of lung tumor initiation and progression using conditional expression of oncogenic K-ras. Genes Dev 15, 3243-3248 (2001).

7. Winters, I.P. et al. Multiplexed in vivo homology-directed repair and tumor barcoding enables parallel quantification of Kras variant oncogenicity. Nat Commun 8, 2053 (2017).

8. Guerra, C. et al. Tumor induction by an endogenous K-ras oncogene is highly dependent on cellular context. Cancer Cell 4, 111-120 (2003).

9. Hingorani, S.R. et al. Preinvasive and invasive ductal pancreatic cancer and its early detection in the mouse. Cancer Cell 4, 437-450 (2003).

10. Zafra, M.P. et al. An In Vivo Kras Allelic Series Reveals Distinct Phenotypes of 559 
11. Paez, J.G. et al. EGFR mutations in lung cancer: correlation with clinical response to gefitinib therapy. Science 304, 1497-1500 (2004).

12. Lynch, T.J. et al. Activating mutations in the epidermal growth factor receptor underlying responsiveness of non-small-cell lung cancer to gefitinib. $N$ Engl $J$ Med 350, 2129-2139 (2004).

13. Politi, K. et al. Lung adenocarcinomas induced in mice by mutant EGF receptors found in human lung cancers respond to a tyrosine kinase inhibitor or to downregulation of the receptors. Genes Dev 20, 1496-1510 (2006).

14. Li, D. et al. Bronchial and peripheral murine lung carcinomas induced by T790ML858R mutant EGFR respond to $\mathrm{HKI}-272$ and rapamycin combination therapy. Cancer Cell 12, 81-93 (2007).

15. Ji, $\mathrm{H}$. et al. The impact of human EGFR kinase domain mutations on lung tumorigenesis and in vivo sensitivity to EGFR-targeted therapies. Cancer Cell 9, 485-495 (2006).

16. Zhu, H. et al. Oncogenic EGFR signaling cooperates with loss of tumor suppressor gene functions in gliomagenesis. Proc Natl Acad Sci U S A 106, 2712-2716 (2009).

17. Lin, Q. et al. The association between BRAF mutation class and clinical features in BRAF-mutant Chinese non-small cell lung cancer patients. J Trans/ Med 17, 298 (2019).

18. van Veen, J.E. et al. Mutationally-activated PI3'-kinase-alpha promotes dedifferentiation of lung tumors initiated by the BRAF(V600E) oncoprotein kinase. Elife 8 (2019).

19. Dankort, D. et al. A new mouse model to explore the initiation, progression, and therapy of BRAFV600E-induced lung tumors. Genes Dev 21, 379-384 (2007).

20. Dankort, D. et al. Braf(V600E) cooperates with Pten loss to induce metastatic melanoma. Nat Genet 41, 544-552 (2009).

21. Charles, R.P., lezza, G., Amendola, E., Dankort, D. \& McMahon, M. Mutationally activated BRAF(V600E) elicits papillary thyroid cancer in the adult mouse. Cancer Res 71, 3863-3871 (2011).

22. Davies, H. et al. Mutations of the BRAF gene in human cancer. Nature 417, 949954 (2002).

23. Chin, L. et al. Essential role for oncogenic Ras in tumour maintenance. Nature 400, 468-472 (1999).

24. Seeburg, P.H., Colby, W.W., Capon, D.J., Goeddel, D.V. \& Levinson, A.D. Biological properties of human c-Ha-ras1 genes mutated at codon 12. Nature 312, 71-75 (1984).

25. Der, C.J., Finkel, T. \& Cooper, G.M. Biological and biochemical properties of human rasH genes mutated at codon 61. Cell 44, 167-176 (1986).

26. Kwong, L.N. et al. Oncogenic NRAS signaling differentially regulates survival and proliferation in melanoma. Nat Med 18, 1503-1510 (2012).

27. Ackermann, J. et al. Metastasizing melanoma formation caused by expression of activated N-RasQ61K on an INK4a-deficient background. Cancer Res 65, 40054011 (2005). 
604 28. Paik, P.K. et al. Response to MET inhibitors in patients with stage IV lung

605

606

607

608

609

610

611

612

613

614

615

616

617

618

619

620

621

622

623

624

625

626

627

628

629

630

631

632

633

634

635

636

637

638

639

640

641

642

643

644

645

646

647

648

649 adenocarcinomas harboring MET mutations causing exon 14 skipping. Cancer Discov 5, 842-849 (2015).

29. Drilon, A. et al. Antitumor activity of crizotinib in lung cancers harboring a MET exon 14 alteration. Nat Med 26, 47-51 (2020).

30. Kim, M. et al. Patient-derived lung cancer organoids as in vitro cancer models for therapeutic screening. Nat Commun 10, 3991 (2019).

31. Gow, C.H. et al. Oncogenic Function of a KIF5B-MET Fusion Variant in NonSmall Cell Lung Cancer. Neoplasia 20, 838-847 (2018).

32. Gao, Y. et al. Allele-Specific Mechanisms of Activation of MEK1 Mutants Determine Their Properties. Cancer Discov 8, 648-661 (2018).

33. Cai, D., Choi, P.S., Gelbard, M. \& Meyerson, M. Identification and Characterization of Oncogenic SOS1 Mutations in Lung Adenocarcinoma. Mol Cancer Res 17, 1002-1012 (2019).

34. Shaw, A.T. et al. Crizotinib versus chemotherapy in advanced ALK-positive lung cancer. N Engl J Med 368, 2385-2394 (2013).

35. Maddalo, D. et al. In vivo engineering of oncogenic chromosomal rearrangements with the CRISPR/Cas9 system. Nature 516, 423-427 (2014).

36. Pyo, K.H. et al. Establishment of a Conditional Transgenic Mouse Model Recapitulating EML4-ALK-Positive Human Non-Small Cell Lung Cancer. J Thorac Oncol 12, 491-500 (2017).

37. Chiarle, R. et al. NPM-ALK transgenic mice spontaneously develop T-cell lymphomas and plasma cell tumors. Blood 101, 1919-1927 (2003).

38. Soda, M. et al. Identification of the transforming EML4-ALK fusion gene in nonsmall-cell lung cancer. Nature 448, 561-566 (2007).

39. Subbiah, V. et al. Precision Targeted Therapy with BLU-667 for RET-Driven Cancers. Cancer Discov 8, 836-849 (2018).

40. Huang, Q. et al. Preclinical Modeling of KIF5B-RET Fusion Lung Adenocarcinoma. Mol Cancer Ther 15, $2521-2529$ (2016).

41. Saito, M. et al. A mouse model of KIF5B-RET fusion-dependent lung tumorigenesis. Carcinogenesis 35, 2452-2456 (2014).

42. Shaw, A.T. et al. Crizotinib in ROS1-rearranged non-small-cell lung cancer. $N$ Engl J Med 371, 1963-1971 (2014).

43. Arai, Y. et al. Mouse model for ROS1-rearranged lung cancer. PLoS One 8, e56010 (2013).

44. Inoue, M. et al. Mouse models for ROS1-fusion-positive lung cancers and their application to the analysis of multikinase inhibitor efficiency. Carcinogenesis 37 , 452-460 (2016).

45. Farago, A.F. et al. Durable Clinical Response to Entrectinib in NTRK1Rearranged Non-Small Cell Lung Cancer. J Thorac Oncol 10, 1670-1674 (2015).

46. Vaishnavi, A. et al. Oncogenic and drug-sensitive NTRK1 rearrangements in lung cancer. Nat Med 19, 1469-1472 (2013).

47. Drilon, A. et al. Response to ERBB3-Directed Targeted Therapy in NRG1Rearranged Cancers. Cancer Discov 8, 686-695 (2018).

48. Hyman, D.M. et al. AKT Inhibition in Solid Tumors With AKT1 Mutations. J Clin Oncol 35, 2251-2259 (2017). 
650 49. De Marco, C. et al. Mutant AKT1-E17K is oncogenic in lung epithelial cells. Oncotarget 6, 39634-39650 (2015).

652 50. Berger, A.H. et al. Oncogenic RIT1 mutations in lung adenocarcinoma.

653

654 Oncogene 33, 4418-4423 (2014).

51. Pillai, R.N. et al. HER2 mutations in lung adenocarcinomas: A report from the
Lung Cancer Mutation Consortium. Cancer 123, 4099-4105 (2017).

52. Perera, S.A. et al. HER2YVMA drives rapid development of adenosquamous lung tumors in mice that are sensitive to BIBW2992 and rapamycin combination therapy. Proc Natl Acad Sci U S A 106, 474-479 (2009).

660

53. Liu, S. et al. Targeting HER2 Aberrations in Non-Small Cell Lung Cancer with Osimertinib. Clin Cancer Res 24, 2594-2604 (2018).

54. Weinstein, E.J., Kitsberg, D.I. \& Leder, P. A mouse model for breast cancer induced by amplification and overexpression of the neu promoter and transgene. Mol Med 6, 4-16 (2000).

55. Guy, C.T. et al. Expression of the neu protooncogene in the mammary epithelium of transgenic mice induces metastatic disease. Proc Natl Acad Sci U S A 89, 10578-10582 (1992).

56. Greulich, H. et al. Functional analysis of receptor tyrosine kinase mutations in lung cancer identifies oncogenic extracellular domain mutations of ERBB2. Proc Natl Acad Sci U S A 109, 14476-14481 (2012).

57. Engelman, J.A. et al. Effective use of PI3K and MEK inhibitors to treat mutant Kras G12D and PIK3CA H1047R murine lung cancers. Nat Med 14, 1351-1356 (2008).

58. Trejo, C.L. et al. Mutationally activated PIK3CA(H1047R) cooperates with BRAF(V600E) to promote lung cancer progression. Cancer Res 73, 6448-6461 (2013).

59. Devarakonda, S. et al. Genomic Profiling of Lung Adenocarcinoma in NeverSmokers. J Clin Oncol, JCO2101691 (2021).

60. Sanchez-Vega, F. et al. Oncogenic Signaling Pathways in The Cancer Genome Atlas. Cell 173, 321-337 e310 (2018).

61. Bailey, M.H. et al. Comprehensive Characterization of Cancer Driver Genes and Mutations. Cell 173, 371-385 e318 (2018).

62. Yi, K.H. \& Lauring, J. Recurrent AKT mutations in human cancers: functional consequences and effects on drug sensitivity. Oncotarget 7, 4241-4251 (2016).

63. Dobashi, Y. et al. Diverse involvement of isoforms and gene aberrations of Akt in human lung carcinomas. Cancer Sci 106, 772-781 (2015).

64. Arboleda, M.J. et al. Overexpression of AKT2/protein kinase Bbeta leads to upregulation of beta1 integrins, increased invasion, and metastasis of human breast and ovarian cancer cells. Cancer Res 63, 196-206 (2003).

65. Zhang, Y. et al. A Pan-Cancer Proteogenomic Atlas of PI3K/AKT/mTOR Pathway Alterations. Cancer Cell 31, 820-832 e823 (2017).

66. Chaft, J.E. et al. Coexistence of PIK3CA and other oncogene mutations in lung adenocarcinoma-rationale for comprehensive mutation profiling. Mol Cancer Ther 11, 485-491 (2012).

67. Dbouk, H.A. et al. Characterization of a tumor-associated activating mutation of the p110beta PI 3-kinase. PLoS One 8, e63833 (2013). 
696

697

698

699

700

701

702

703

704

705

706

707

708

709

710

711

712

713

714

715

716

717

718

719

720

721

722

723

724

725

726

727

728

729

730

731

732

733

734

735

736

737

738

739

68. Pazarentzos, E. et al. Oncogenic activation of the PI3-kinase p110beta isoform via the tumor-derived PIK3Cbeta(D1067V) kinase domain mutation. Oncogene 35, 1198-1205 (2016).

69. Whale, A.D., Colman, L., Lensun, L., Rogers, H.L. \& Shuttleworth, S.J. Functional characterization of a novel somatic oncogenic mutation of PIK3CB. Signal Transduct Target Ther 2, 17063 (2017).

70. Liyasova, M.S., Ma, K. \& Lipkowitz, S. Molecular pathways: cbl proteins in tumorigenesis and antitumor immunity-opportunities for cancer treatment. Clin Cancer Res 21, 1789-1794 (2015).

71. Maertens, O. \& Cichowski, K. An expanding role for RAS GTPase activating proteins (RAS GAPs) in cancer. Adv Biol Regul 55, 1-14 (2014).

72. Ahmad, M.K., Abdollah, N.A., Shafie, N.H., Yusof, N.M. \& Razak, S.R.A. Dualspecificity phosphatase 6 (DUSP6): a review of its molecular characteristics and clinical relevance in cancer. Cancer Biol Med 15, 14-28 (2018).

73. Owens, D.M. \& Keyse, S.M. Differential regulation of MAP kinase signalling by dual-specificity protein phosphatases. Oncogene 26, 3203-3213 (2007).

74. Sato, K. et al. Fusion Kinases Identified by Genomic Analyses of Sporadic Microsatellite Instability-High Colorectal Cancers. Clin Cancer Res 25, 378-389 (2019).

75. Nonami, A. et al. Spred-1 negatively regulates interleukin-3-mediated ERK/mitogen-activated protein (MAP) kinase activation in hematopoietic cells. $J$ Biol Chem 279, 52543-52551 (2004).

76. McClatchey, A.I. \& Cichowski, K. SPRED proteins provide a NF-ty link to Ras suppression. Genes Dev 26, 1515-1519 (2012).

77. Hanafusa, H., Torii, S., Yasunaga, T. \& Nishida, E. Sprouty1 and Sprouty2 provide a control mechanism for the Ras/MAPK signalling pathway. Nat Cell Biol 4, 850-858 (2002).

78. Yusoff, P. et al. Sprouty2 inhibits the Ras/MAP kinase pathway by inhibiting the activation of Raf. J Biol Chem 277, 3195-3201 (2002).

79. Kim, H.J. \& Bar-Sagi, D. Modulation of signalling by Sprouty: a developing story. Nat Rev Mol Cell Biol 5, 441-450 (2004).

80. Chakravarty, D. et al. OncoKB: A Precision Oncology Knowledge Base. JCO Precis Oncol 2017 (2017).

81. Liu, C. et al. Mosaic analysis with double markers reveals tumor cell of origin in glioma. Cell 146, 209-221 (2011).

82. Zhu, Y. et al. Ablation of NF1 function in neurons induces abnormal development of cerebral cortex and reactive gliosis in the brain. Genes Dev 15, 859-876 (2001).

83. Madisen, L. et al. A robust and high-throughput Cre reporting and characterization system for the whole mouse brain. Nat Neurosci 13, 133-140 (2010).

84. Chiou, S.H. et al. Pancreatic cancer modeling using retrograde viral vector delivery and in vivo CRISPR/Cas9-mediated somatic genome editing. Genes Dev 29, 1576-1585 (2015). 
782

783

85. Okawa, H. et al. Hepatocyte-specific deletion of the keap1 gene activates Nrf2 and confers potent resistance against acute drug toxicity. Biochem Biophys Res Commun 339, 79-88 (2006).

86. Bardeesy, N. et al. Loss of the Lkb1 tumour suppressor provokes intestinal polyposis but resistance to transformation. Nature 419, 162-167 (2002).

87. Jonkers, J. et al. Synergistic tumor suppressor activity of BRCA2 and p53 in a conditional mouse model for breast cancer. Nat Genet 29, 418-425 (2001).

88. Rogers, Z.N. et al. A quantitative and multiplexed approach to uncover the fitness landscape of tumor suppression in vivo. Nat Methods 14, 737-742 (2017).

89. Rogers, Z.N. et al. Mapping the in vivo fitness landscape of lung adenocarcinoma tumor suppression in mice. Nat Genet 50, 483-486 (2018).

90. Cai, H., et al. A functional taxonomy of tumor suppression in oncogenic KRASdriven lung cancer. Under consideration (2021).

91. Murray, C.W. et al. An LKB1-SIK Axis Suppresses Lung Tumor Growth and Controls Differentiation. Cancer Discov 9, 1590-1605 (2019).

92. Orr, H.A. Fitness and its role in evolutionary genetics. Nat Rev Genet 10, 531539 (2009).

93. J.B.S., H. The mathematical theory of natural and artificial selection. Mathematical Proceedings of the Cambridge Philosophical Society 23, 607-615 (1927).

94. Geraldine A. Van der Auwera, B.D.O.C. Genomics in the Cloud. (2020).

95. McLaren, W. et al. The Ensembl Variant Effect Predictor. Genome Biol 17, 122 (2016).

96. Fedchenko, N. \& Reifenrath, J. Different approaches for interpretation and reporting of immunohistochemistry analysis results in the bone tissue - a review. Diagn Pathol 9, 221 (2014).

97. Feoktistova, M., Geserick, P. \& Leverkus, M. Crystal Violet Assay for Determining Viability of Cultured Cells. Cold Spring Harb Protoc 2016, pdb prot087379 (2016).

98. Nichols, R.J. et al. RAS nucleotide cycling underlies the SHP2 phosphatase dependence of mutant BRAF-, NF1- and RAS-driven cancers. Nat Cell Biol 20, 1064-1073 (2018).

99. lanevski, A., Giri, A.K. \& Aittokallio, T. SynergyFinder 2.0: visual analytics of multi-drug combination synergies. Nucleic Acids Res 48, W488-W493 (2020).

100. Loewe, S. The problem of synergism and antagonism of combined drugs. Arzneimittelforschung 3, 285-290 (1953).

101. Dobin, A. et al. STAR: ultrafast universal RNA-seq aligner. Bioinformatics 29, 1521 (2013).

102. Li, B. \& Dewey, C.N. RSEM: accurate transcript quantification from RNA-Seq data with or without a reference genome. BMC Bioinformatics 12, 323 (2011).

103. Soneson, C., Love, M.I. \& Robinson, M.D. Differential analyses for RNA-seq: transcript-level estimates improve gene-level inferences. F1000Res 4, 1521 (2015).

104. Love, M.I., Huber, W. \& Anders, S. Moderated estimation of fold change and dispersion for RNA-seq data with DESeq2. Genome Biol 15, 550 (2014). 
105. Subramanian, A. et al. Gene set enrichment analysis: a knowledge-based approach for interpreting genome-wide expression profiles. Proc Natl Acad Sci U $S$ A 102, 15545-15550 (2005).

788 106. Hanzelmann, S., Castelo, R. \& Guinney, J. GSVA: gene set variation analysis for

789 microarray and RNA-seq data. BMC Bioinformatics 14, 7 (2013). 